TRANSACTIONS OF THE

AMERICAN MATHEMATICAL SOCIETY

Volume 361, Number 8, August 2009, Pages 4299-4365

S 0002-9947(09)04747-3

Article electronically published on March 13, 2009

\title{
LONG-TIME SOBOLEV STABILITY FOR SMALL SOLUTIONS OF QUASI-LINEAR KLEIN-GORDON EQUATIONS ON THE CIRCLE
}

\author{
J.-M. DELORT
}

\begin{abstract}
We prove that higher Sobolev norms of solutions of quasi-linear Klein-Gordon equations with small Cauchy data on $\mathbb{S}^{1}$ remain small over intervals of time longer than the ones given by local existence theory. This result extends previous ones obtained by several authors in the semi-linear case. The main new difficulty one has to cope with is the loss of one derivative coming from the quasi-linear character of the problem. The main tool used to overcome it is a global paradifferential calculus adapted to the Sturm-Liouville operator with periodic boundary conditions.
\end{abstract}

\section{INTRODUCTION}

We address in this paper the question of long-time Sobolev stability for small solutions of nonlinear Klein-Gordon equations on $\mathbb{S}^{1}$. Let us recall some known results. Consider $V: \mathbb{S}^{1} \rightarrow \mathbb{R}$ a smooth nonnegative potential and consider $u$ a solution of the equation

$$
\begin{aligned}
& \frac{\partial^{2} u}{\partial t^{2}}-\frac{\partial^{2} u}{\partial x^{2}}+\left(V(x)+m^{2}\right) u=f(u), \\
& \left.u\right|_{t=0}=\epsilon u_{0}, \\
& \left.\partial_{t} u\right|_{t=0}=\epsilon u_{0},
\end{aligned}
$$

where $\epsilon>0$ is a small parameter, $m \in] 0,+\infty[, f$ is a nonlinearity vanishing at order $\kappa+1 \geq 2$ at 0 . It is well known that such an equation has a unique $C^{0}\left(\mathbb{R}, H^{1}\right) \cap C^{1}\left(\mathbb{R}, L^{2}\right)$ solution if $u_{0} \in H^{1}\left(\mathbb{S}^{1}, \mathbb{R}\right), u_{1} \in L^{2}\left(\mathbb{S}^{1}, \mathbb{R}\right)$ and $\epsilon$ is small enough. The question is to decide whether, when $u_{0} \in H^{s+1}\left(\mathbb{S}^{1}, \mathbb{R}\right), u_{1} \in H^{s}\left(\mathbb{S}^{1}, \mathbb{R}\right)$ $(s \gg 1),\|u(t, \cdot)\|_{H^{s+1}}+\left\|\partial_{t} u(t, \cdot)\right\|_{H^{s}}$ stays bounded over long intervals of time when $\epsilon \rightarrow 0$, i.e. over intervals of length $c \epsilon^{-r+1}$ with $r>\kappa+1$ (the case $r=\kappa+1$ would correspond to the bound given by local existence theory). The difficulty of the problem comes from the fact that on $\mathbb{S}^{1}$ one does not have any dispersion making decay linear solutions at infinite times, in contrast to what happens for that equation on the real line. (We refer to chapter 7 of the book of Hörmander [13] for results and references concerning the nonlinear Klein-Gordon equation on $\mathbb{R}^{d}$, and

Received by the editors September 19, 2007.

2000 Mathematics Subject Classification. Primary 35L70, 35S50.

Key words and phrases. Quasi-linear Klein-Gordon equation, long-time stability, paradifferential calculus.

This work was partially supported by the ANR project Equa-disp.

(C)2009 American Mathematical Society Reverts to public domain 28 years from publication 
to Shatah [15] for the first occurrence in this setting of the normal form method that will play an essential role below.)

Bourgain answered the above question for equation (0.0.1) in [5]. He showed that the solutions remain bounded in $H^{s+1} \times H^{s}$ for intervals of time of length $c \epsilon^{-N}$ for any $N$, when $s \gg N$, and when the parameter $m$ in (0.0.1) is taken outside a subset

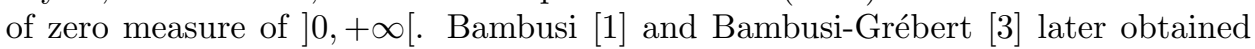
more precise versions of this result (see also the lectures notes of Grébert [12). Let us mention that, as far as we know, there is no example of solutions which, when $m$ is in the exceptionnal set excluded in the above result, would have an $H^{s+1} \times H^{s}$ norm blowing up when time goes to infinity. Nevertheless, Bourgain 6 constructed an example of an abstract perturbation of the linear wave equation for which such a blowing-up property occurs.

Two natural questions arise: can such results be extended to equations with more general nonlinearities than the one of (0.0.1), and do they hold true in higher dimension? The latter question has been answered affirmatively for equations of type (0.0.1) on the sphere $\mathbb{S}^{d}$, or more generally on Zoll manifolds, by Bambusi, Grébert, Szeftel and the author in [2]. The former question has been taken up in 19, 10, 11, including in higher dimensions, for equations of type 0.0.1) in which the right hand side is replaced by a general semi-linear nonlinearity $f\left(u, \partial_{t} u, \partial_{x} u\right)$. For such nonlinearities, the solution does not in general exist over an interval of time larger than the one given by local existence theory (i.e. ] $-c \epsilon^{-\kappa}, c \epsilon^{\kappa}$ [ if $f$ vanishes at order $\kappa+1$ at zero); see 8 for examples of blowing-up solutions. Nevertheless, a result proved in [9, 10] asserts that if, for instance, $f$ is homogeneous of even degree $\kappa+1$, then the solution of the equation exists and remains bounded in $H^{s+1} \times H^{s}$ over an interval of time of length $c \epsilon^{-2 \kappa}$. The method of proof was similar to the one used by Bourgain [5], Bambusi [1], and Bambusi-Grébert [3], the main novelty being its extension to a higher dimensional setting. Our goal in this paper is to address the same question in one space dimension for quasi-linear Klein-Gordon equations. As we shall explain below, the semi-linear methods of the above papers break down immediately because of the extra loss of one derivative coming from the quasi-linear nature of the problem. Our main theorem is stated in section 1 below. We shall in this introduction describe our method on the example

$$
\begin{aligned}
& \left(D_{t}-(1+a(u, \bar{u})) \sqrt{-\Delta+V+m^{2}}\right) u=0, \\
& \left.u\right|_{t=0}=\epsilon u_{0},
\end{aligned}
$$

where $u_{0}$ is a smooth complex-valued function defined on $\mathbb{S}^{1}, \Delta=\frac{d^{2}}{d x^{2}}$, and $u \rightarrow$ $a(u, \bar{u})$ is a real-valued polynomial in $(u, \bar{u})$, homogeneous of odd degree $\kappa$. Our aim is to prove existence of the solution, and uniform control of its $H^{s}$-norm $(s \gg 1)$ by $C \epsilon$, over an interval of time of length $c \epsilon^{-2 \kappa}$ (instead of the length $c \epsilon^{-\kappa}$ given by local existence theory). Let us first recall how the corresponding semi-linear result may be proved. Let us take, for simplicity, the case $V \equiv 0$ and consider

$$
\begin{aligned}
& \left(D_{t}-\sqrt{-\Delta+m^{2}}\right) u=f(u, \bar{u}), \\
& \left.u\right|_{t=0}=\epsilon u_{0},
\end{aligned}
$$

where $f(u, \bar{u})=u^{p} \bar{u}^{q}$ with $p+q=\kappa+1$. Set $\Lambda_{m}=\sqrt{-\Delta+m^{2}}, \Lambda=\sqrt{-\Delta+1}$ and let $\Pi_{n}$ be the spectral projector on the space generated by the eigenfunctions $e^{ \pm i n x}(n \in \mathbb{N})$. Then the $H^{s}$ norm is given by $\|u\|_{H^{s}}^{2}=\left\langle\Lambda^{s} u, \Lambda^{s} u\right\rangle=$ 
$\sum_{n=0}^{+\infty}\left(1+n^{2}\right)^{s}\left\|\Pi_{n} u\right\|_{L^{2}}^{2}$ and if $u$ solves (0.0.3), then

$$
\frac{1}{2} \frac{d}{d t}\|u(t, \cdot)\|_{H^{s}}^{2}=-\operatorname{Im}\left[\left\langle\Lambda^{s}\left(\Lambda_{m} u\right), \Lambda^{s} u\right\rangle+\left\langle\Lambda^{s} f(u, \bar{u}), \Lambda^{s} u\right\rangle\right] .
$$

The first term on the right hand side vanishes by selfadjointness of $\Lambda_{m}$, and the second one may be written $-\operatorname{Im} M_{0}(u, \ldots, \bar{u})$ with

$$
\begin{aligned}
& M_{0}(\underbrace{u, \ldots, u}_{p}, \underbrace{\bar{u}, \ldots, \bar{u}}_{q+1}) \\
& \quad=\sum_{n_{1}, \ldots, n_{p+q+1}}\left(1+n_{p+q+1}^{2}\right)^{s} \int_{\mathbb{S}^{1}} \Pi_{n_{1}} u \cdots \Pi_{n_{p}} u \overline{\Pi_{n_{p+1}} u} \cdots \overline{\Pi_{n_{p+q+1}} u} d x .
\end{aligned}
$$

The idea of the method is to perturb the $H^{s}$ energy of $u$ by a multilinear expression

$$
\operatorname{Re} M_{1}(\underbrace{u, \ldots, u, \bar{u}, \ldots, \bar{u}}_{p+q+1=\kappa+2})
$$

such that $\frac{d}{d t} M_{1}(u, \ldots, \bar{u})$ will cancel out (0.0.5) up to a remainder which will be $O\left(\|u\|_{H^{s}}^{2 \kappa+2}\right)$. This gain on the order of vanishing at 0 , versus the one of the last term in (0.0.4), allows one to obtain the longer interval of time $c \epsilon^{-2 \kappa}$ by standard arguments. Using (0.0.3), one finds that

$$
\frac{d}{d t} M_{1}(u, \ldots, u, \bar{u}, \ldots, \bar{u})=i L\left(M_{1}\right)(u, \ldots, u, \bar{u}, \ldots, \bar{u})+R(u, \bar{u}),
$$

where

$$
\begin{aligned}
& L\left(M_{1}\right)(u, \ldots, \bar{u})=\sum_{1}^{p} M_{1}\left(u, \ldots, \Lambda_{m} u, \ldots, u, \bar{u}, \ldots, \bar{u}\right) \\
&-\sum_{p+1}^{p+q+1} M_{1}\left(u, \ldots, u, \bar{u}, \ldots, \Lambda_{m} \bar{u}, \ldots, \bar{u}\right),
\end{aligned}
$$

and $R(u, \bar{u})$ is a remainder obtained by substituting if $(u, \bar{u})$ to one of the arguments of $M_{1}$. Since $f$ contains no derivative of $u, R(u, \bar{u})=O\left(\|u\|_{H^{s}}^{2 \kappa+2}\right)$ as wanted. As $\Lambda_{m} \Pi_{n} u=\sqrt{m^{2}+n^{2}} \Pi_{n} u$, one may write

$$
\begin{aligned}
& L\left(M_{1}\right)\left(\Pi_{n_{1}} u_{1}, \ldots, \Pi_{n_{p+q+1}} u_{p+q+1}\right) \\
& \quad=F_{m}\left(n_{1}, \ldots, n_{p+q+1}\right) M_{1}\left(\Pi_{n_{1}} u_{1}, \ldots, \Pi_{n_{p+q+1}} u_{p+q+1}\right),
\end{aligned}
$$

where we denoted

$$
F_{m}\left(n_{1}, \ldots, n_{p+q+1}\right)=\sum_{1}^{p} \sqrt{m^{2}+n_{j}^{2}}-\sum_{p+1}^{p+q+1} \sqrt{m^{2}+n_{j}^{2}} .
$$

To eliminate in $\frac{d}{d t}\left[\frac{1}{2}\|u(t, \cdot)\|_{H^{s}}^{2}+\operatorname{Re} M_{1}(u, \ldots, \bar{u})\right]$ terms homogeneous of degree $\kappa+1$, one has to choose $M_{1}$ so that $L\left(M_{1}\right)=-M_{0}$; i.e. according to (0.0.8) and (0.0.5),

(0.0.10)

$$
\begin{aligned}
& M_{1}\left(\Pi_{n_{1}} u_{1}, \ldots, \Pi_{n_{p+q+1}} u_{p+q+1}\right) \\
& \quad=-F_{m}\left(n_{1}, \ldots, n_{p+q+1}\right)^{-1}\left(1+n_{p+q+1}^{2}\right)^{s} \int_{\mathbb{S}^{1}} \Pi_{n_{1}} u_{1} \cdots \Pi_{n_{p+q+1}} u_{p+q+1} d x .
\end{aligned}
$$


Since $p+q$ is even, it may be proved that for $m$ outside an exceptionnal subset of zero measure, $F_{m}\left(n_{1}, \ldots, n_{p+q+1}\right)$ does not vanish, and actually

$$
\left|F_{m}\left(n_{1}, \ldots, n_{p+q+1}\right)\right|^{-1} \leq C \mu\left(n_{1}, \ldots, n_{p+q+1}\right)^{N_{0}}
$$

for some $N_{0}, \mu\left(n_{1}, \ldots, n_{p+q+1}\right)$ standing for the third largest among $n_{1}, \ldots, n_{p+q+1}$. This shows that $\left|F_{m}\right|^{-1}$ is bounded from above by a power of a small frequency, which allows one to prove, combining this with convenient estimates of the integral in (0.0.10), that $M_{1}$ is a continuous multilinear form on $H^{s} \times \cdots \times H^{s}$ for $s \gg N_{0}$, and so a small perturbation of the $H^{s}$ energy when $u$ is small. Let us notice that related ideas are used for problems on $\mathbb{R}^{n}$ by Colliander, Keel, Staffilani, Takaoka and Tao in [7.

Let us go back to the quasi-linear equation (0.0.2). In this case (0.0.4) can be written as

$$
\begin{aligned}
\frac{1}{2} \frac{d}{d t}\|u(t, \cdot)\|_{H^{s}}^{2} & =-\operatorname{Im}\left\langle\Lambda^{s} a(u, \bar{u}) \Lambda_{m} u, \Lambda^{s} u\right\rangle \\
& =\frac{1}{2 i}\left\langle\Lambda^{s}\left[\Lambda^{-2 s} \Lambda_{m}, a \Lambda^{2 s}\right] u, \Lambda^{s} u\right\rangle .
\end{aligned}
$$

Since the operator $\left[\Lambda^{-2 s} \Lambda_{m}, a \Lambda^{2 s}\right]$ is of order 0 , we still get a quantity well defined on $H^{s}$, even if its expression is now a little bit more complicated than (0.0.5). We would like to argue as above and find a new contribution $\operatorname{Re} M_{1}$ to add to $\frac{1}{2}\|u(t, \cdot)\|_{H^{s}}^{2}$, so that its time derivative would cancel out the right hand side of (0.0.11), up to remainders. The $R(u, \bar{u})$ terms in (0.0.6) would be given by

$$
\begin{aligned}
R(u, \bar{u})= & i\left(\sum_{1}^{p} M_{1}\left(u, \ldots, u, a(u, \bar{u}) \Lambda_{m} u, u, \ldots, u, \bar{u}, \ldots, \bar{u}\right)\right. \\
& \left.\quad-\sum_{p+1}^{p+q+1} M_{1}\left(u, \ldots, u, \bar{u}, \ldots, \bar{u}, a(u, \bar{u}) \Lambda_{m} \bar{u}, \bar{u}, \ldots, \bar{u}\right)\right) .
\end{aligned}
$$

This quantity is no longer of order 0 in $u, \bar{u}$ for a general $M_{1}$, which means that $R(u, \bar{u})$ could no longer be estimated by $C\|u\|_{H^{s}}^{2 \kappa+2}$ but only by $C\|u\|_{H^{s}}^{2 \kappa+1}\|u\|_{H^{s+1}}$. This loss of derivative, which is systematic in quasi-linear problems, cannot be recovered if $M_{1}$ is a multilinear form which does not satisfy any structure condition. On the other hand, if we know that $M_{1}$ has a structure similar to the quantity on the right hand side of (0.0.11), we may hope to make appear a commutator that will kill the extra loss of one derivative. This is actually the usual way of getting quasi-linear energy inequalities. The price we have to pay to be able to do so is that we must get for $M_{0}, M_{1}$ expressions more explicit than just multilinear quantities satisfying convenient estimates, like those used in the semilinear problems treated in the aforementionned references. We must be able to write $M_{0}$ or $M_{1}$ as

$$
\langle\mathrm{Op}(c(u, \ldots, \bar{u} ; \cdot)) u, u\rangle,
$$

where $c\left(u_{1}, \ldots, u_{p} ; \cdot\right)$ will be a convenient paradifferential symbol that may be computed from the equation, and $\operatorname{Op}(c)$ is the operator associated to that symbol. The difficulty that arises is the following: we must work globally on $\mathbb{S}^{1}$, and we cannot restrict ourselves to open subsets of $\mathbb{R}$ through local charts. This is because our class of symbols will have to contain functions defined in terms of $F_{m}\left(n_{1}, \ldots, n_{p+q+1}\right)^{-1}$, where $F_{m}$ is given in (0.0.9) (to be able to construct the analogue of $M_{1}$; see (0.0.10) ). This quantity is well defined for $m$ outside an exceptional subset, only 
when the arguments $n_{1}, \ldots, n_{p+q+1}$ stay in a discrete set. In other words, we cannot use Bony's calculus of paradifferential operators on $\mathbb{R}[4$, since their symbols are functions of a continuous phase variable. We must instead define a global paradifferential calculus on $\mathbb{S}^{1}$, in terms of symbols whose phase variable varies in the (discrete) spectrum of $-\frac{d^{2}}{d x^{2}}+V$ on $\mathbb{S}^{1}$. When $V \equiv 0$, this is done through Fourier series expansions. An example of the type of symbols we have to consider is given by

$$
\left(n_{0}, n_{1}\right) \rightarrow\left\langle a e^{i n_{0} x}, e^{i n_{1} x}\right\rangle=\hat{a}\left(n_{1}-n_{0}\right),
$$

where $a \in C^{\infty}\left(\mathbb{S}^{1}\right)$. Such a quantity is rapidly decaying in $n_{0}-n_{1}$, and its $\partial_{n_{0}}+\partial_{n_{1}}$ derivative vanishes. In general, when $V \not \equiv 0$, the class of symbols we want to consider has to include quantities like

$$
\left(n_{0}, n_{1}\right) \rightarrow\left\langle a \varphi_{n_{0}}, \varphi_{n_{1}}\right\rangle,
$$

where $\varphi_{n_{0}}, \varphi_{n_{1}}$ are two eigenfunctions, and we want them to verify estimates of form

$$
\left|\left(\partial_{n_{0}}+\partial_{n_{1}}\right)^{\gamma}\left\langle a \varphi_{n_{0}}, \varphi_{n_{1}}\right\rangle\right| \leq C_{N}\left\langle n_{0}-n_{1}\right\rangle^{-N}\left(n_{0}+n_{1}\right)^{-\gamma} .
$$

The first section of this paper is devoted to the construction of a nice basis of $L^{2}\left(\mathbb{S}^{1}\right)$, i.e. of an orthonormal basis of almost eigenfunctions for which estimates of the form (0.0.13) hold true. This is done using quasi-modes for $-\frac{d^{2}}{d x^{2}}+V$ which resemble the imaginary exponentials of the free case.

The second section of the paper is devoted to the definition of paradifferential operators associated to symbols whose phase argument varies in a discrete set. We establish the main symbolic calculus properties of such operators.

The third section presents a special class of pseudo-differential operators, containing the operators involved in the writing of equation 0.0 .1 . These special operators enjoy more explicit symbolic calculus properties than the general ones defined in section 2 .

The fourth section is devoted to the proof of the theorem, using the machinery of sections 2 and 3 to be able to get the energy estimates we alluded to at the beginning of this introduction. We first perform a paradifferential diagonalization of the principal part of the wave operator, reducing (0.0.1) to a paradifferential version of (0.0.2). We then apply the energy method, as explained after (0.0.11). The fact that we reduced ourselves to a diagonal principal symbol, together with the symbolic calculus constructed in the preceding sections, allows us to show that the remainders of the form (0.0.12) that we get actually involve commutators compensating the apparent loss of one derivative displayed by 0.0 .11 . In that way, we are able to obtain energy inequalities of type $\frac{d}{d t}\|u(t, \cdot)\|_{H^{s}}^{2} \leq C\|u(t, \cdot)\|_{H^{s}}^{2 \kappa+2}$, which imply the long-time existence result we are looking for.

Let us conclude this introduction by expressing our gratitude to Dario Bambusi for several conversations about this work. Let us say also that we shall use in the text the following notation: we write $n_{0} \sim n_{1}$ to mean that there is a (large) constant $C>0$ with $C^{-1} n_{0} \leq n_{1} \leq C n_{0}$ when $n_{0}, n_{1} \rightarrow+\infty$, and we set $n_{0} \ll n_{1}$ to say that there is a small $c>0$ with $n_{0} \leq c n_{1}$ when $n_{0}, n_{1} \rightarrow+\infty$.

\section{MAin RESUlts AND NICE BASIS}

1.1. Statement of main theorem. We shall be interested in this paper in solutions of the periodic one dimensional quasi-linear Klein-Gordon equation. We 
denote by $\Delta=\frac{d^{2}}{d x^{2}}$ the Laplace operator on $\mathbb{S}^{1}$, and take $V: \mathbb{S}^{1} \rightarrow \mathbb{R}_{+}$a smooth nonnegative potential. We shall sometimes identify $\mathbb{S}^{1}$ with the interval $[-\pi, \pi]$ with periodic boundary conditions. We consider a polynomial map

$$
\begin{aligned}
c: \mathbb{R}^{3} & \longrightarrow \mathbb{R}, \\
\left(X_{0}, X_{1}, X_{2}\right) & \longrightarrow c\left(X_{0}, X_{1}, X_{2}\right),
\end{aligned}
$$

which may be written as

$$
c\left(X_{0}, X_{1}, X_{2}\right)=\sum_{k=\kappa}^{\kappa_{1}} c_{k}\left(X_{0}, X_{1}, X_{2}\right)
$$

where $c_{k}$ is homogeneous of degree $k$ in $\left(X_{0}, X_{1}, X_{2}\right)$. We denote by $r$ the largest odd integer satisfying $\kappa \leq r-1 \leq 2 \kappa$ and

for any even integer $2 k$ satisfying $\kappa \leq 2 k<r-1$, one has $c_{2 k}\left(X_{0}, X_{1}, X_{2}\right) \equiv 0$.

We shall consider the following equation, where $m>0$ is a parameter:

$$
\begin{aligned}
& \partial_{t}^{2} v+\left(1+c\left(v, \partial_{t} v, \partial_{x} v\right)\right)^{2}\left[-\Delta+V+m^{2}\right] v=0, \\
& \left.v\right|_{t=0}=\epsilon v_{0}, \\
& \left.\partial_{t} v\right|_{t=0}=\epsilon v_{1},
\end{aligned}
$$

where $v_{0}$ and $v_{1}$ are smooth real-valued functions defined on $\mathbb{S}^{1}$, and $\epsilon>0$ is a small parameter. Our main result is the following:

Theorem 1.1.1. There is a zero measure subset $\mathcal{N}$ of $] 0,+\infty[$, and for every $m \in$ $\mathcal{N}$, there are $c>0, s_{0} \in \mathbb{N}$, such that for any $s \geq s_{0}$, any $\left(v_{0}, v_{1}\right) \in H^{s+1}\left(\mathbb{S}^{1}, \mathbb{R}\right) \times$ $H^{s}\left(\mathbb{S}^{1}, \mathbb{R}\right)$, satisfying for $\left.\epsilon \in\right] 0,1[$,

$$
\left\|v_{0}\right\|_{H^{s_{0}+1}}+\left\|v_{1}\right\|_{H^{s_{0}}}<\epsilon
$$

equation (1.1.4) has a unique solution

$$
v \in C^{0}(]-T_{\epsilon}, T_{\epsilon}\left[, H^{s+1}\left(\mathbb{S}^{1}, \mathbb{R}\right)\right) \cap C^{1}(]-T_{\epsilon}, T_{\epsilon}\left[, H^{s}\left(\mathbb{S}^{1}, \mathbb{R}\right)\right)
$$

with $T_{\epsilon} \geq c \epsilon^{-r+1}$. Moreover, there is for any $s \geq s_{0}$ a constant $c_{s}>0$, such that if $\left(v_{0}, v_{1}\right)$ satisfies (1.1.5) with $s_{0}$ replaced by $s,\|v(t, \cdot)\|_{H^{s+1}}+\left\|\partial_{t} v(t, \cdot)\right\|_{H^{s}}$ is uniformly bounded on the interval $]-T_{\epsilon}^{\prime}, T_{\epsilon}^{\prime}\left[\right.$ with $T_{\epsilon}^{\prime} \geq c_{s} \epsilon^{-r+1}$.

Remarks. - It is enough to prove that for $s_{0}$ large enough, condition (1.1.5) with $\epsilon>0$ small enough implies the existence of an $H^{s_{0}+1} \times H^{s_{0}}$ bounded solution defined on $]-T_{\epsilon}, T_{\epsilon}\left[\times \mathbb{S}^{1}\right.$. We know then that if the Cauchy data $\left(v_{0}, v_{1}\right)$ belong to $H^{s+1} \times H^{s}$ with $s \geq s_{0}$, their smoothness will be propagated by the equation.

- The time of existence given by local existence theory is $c \epsilon^{-\kappa}$. If $\kappa$ is even and $c_{\kappa} \not \equiv 0$ in (1.1.2), then (1.1.3) gives $r=\kappa+1$, and the theorem is empty: it just asserts that there is a solution defined on the interval of time given by local existence theory. Because of that, we shall assume in the sequel that $\kappa$ is odd.

- If $\kappa$ is odd, and $c_{2 k} \equiv 0$ if $\kappa<2 k<2 \kappa$, we may take $r=2 \kappa+1$, and we get a solution on an interval of length $\epsilon^{-2 \kappa}$, i.e. on a much larger interval than the one given by local existence theory.

- In the semi-linear case, Theorem 1.1.1 has been proved (with more general assumptions on the nonlinearity) in [9, 10, when the equation is posed more generally on $\mathbb{S}^{d}$, or on a Zoll manifold of any dimension. 
- For semi-linear equations on Zoll manifolds, whose nonlinearities depend only on $v$, and not on its derivatives, it has been proved in 22 that the solution of the problem is almost global, i.e. defined on intervals of length $c_{N} \epsilon^{-N}$ for any $N$. Moreover one has uniform Sobolev estimates on such intervals. This result had been obtained previously in one dimension by Bourgain [5], on a slightly weaker form, and by Bambusi [1] and Bambusi-Grébert [3].

- In the quasi-linear case, no result seems to have been known, except in the much simpler case of equations of the form (1.1.4) with zero potential and a quadratic nonlinearity on $\mathbb{T}^{d}(d \geq 1)$; see [9. For such operators and nonlinearities, most of the difficulties we shall encounter in this paper disappear. Actually, the fact that the potential is zero allows one to use Fourier series, and so harmonic analysis. The combination of this and of the fact that the nonlinearity is quadratic makes functions of type (0.0.9) always nonzero whatever the value of the parameter $m$ on the relevant set of arguments. Because of that, the proof does not use the structure of the spectrum of the Laplacian, and this explains why one is able to treat also the case of tori of higher dimension. On the other hand, as soon as either the potential is nonzero, or the nonlinearity vanishes at order strictly larger than two, the structure of the spectrum plays an essential role. This explains why, in such cases, no result is known on $\mathbb{T}^{d}(d \geq 2)$, even for semi-linear equations.

- A natural question is to know if Theorem 1.1.1 may be extended from $\mathbb{S}^{1}$ to $\mathbb{S}^{d}$, as its semi-linear counterpart. We are unable to perform such an extension. This is related to the existence of a "nice basis", which will be addressed in the next subsection.

1.2. Nice basis. Let $V: \mathbb{S}^{1} \rightarrow \mathbb{R}_{+}$be a smooth function. The large eigenvalues of $-\frac{d^{2}}{d x^{2}}+V$ are arranged in couples $\left(\omega_{n}^{-}\right)^{2} \leq\left(\omega_{n}^{+}\right)^{2}$, where $\omega_{n}^{+}$and $\omega_{n}^{-}$have when $n \rightarrow+\infty$ a same asymptotic expansion at any order of the form

$$
n+\frac{1}{4 \pi n} \int_{\mathbb{S}^{1}} V(x) d x+\frac{\alpha_{3}}{n^{3}}+\frac{\alpha_{5}}{n^{5}}+\cdots
$$

(see for instance the book of Marchenko 14]). We shall denote in this subsection for $n$ large enough by $E_{n}$ the subspace of $L^{2}\left(\mathbb{S}^{1}, \mathbb{R}\right)$ spanned by the eigenfunctions associated to $\left(\omega_{n}^{-}\right)^{2}$ and $\left(\omega_{n}^{+}\right)^{2}$, and by $\Pi_{n}$ the spectral projection of $L^{2}$ onto that subspace. We shall choose a function $\lambda \rightarrow \omega(\lambda)$, which is a symbol of order 1 , having when $\lambda \rightarrow+\infty$ the expansion (1.2.1) (with $n$ replaced by $\lambda$ ). If we write $a_{n}=O\left(n^{-\infty}\right)$ to mean that for any $N \in \mathbb{N}$ there is $C_{N}>0$ with $\left|a_{n}\right| \leq C_{N} n^{-N}$, then $\omega(n)-\omega_{n}^{ \pm}=O\left(n^{-\infty}\right)$. Consequently, we have

$$
\left\|\sqrt{-\Delta+V} \Pi_{n}-\omega(n) \Pi_{n}\right\|_{\mathcal{L}\left(L^{2}, L^{2}\right)}=O\left(n^{-\infty}\right) .
$$

Our goal is to construct a basis of each $E_{n}$ such that some scalar products involving elements of these bases will have symbolic behaviour relative to the spectral parameters. Before stating the theorem, let us introduce the following notation. For $\tau \in \mathbb{N}$, we denote by $\mathbb{N}_{\tau}=\{n \in \mathbb{N} ; n \geq \tau\}$. If $a: \mathbb{N}_{\tau} \rightarrow \mathbb{C}$ is given, we extend it by 0 to a function defined on $\mathbb{Z}$, and we define $\partial a: \mathbb{N}_{\tau} \rightarrow \mathbb{C}$ by

$$
\partial a(n)=a(n+1)-a(n) .
$$


We denote by $\partial^{*}$ the formal adjoint of $\partial$ for the scalar product $\langle a, b\rangle=\sum_{n \geq \tau} a(n) \overline{b(n)}$, that is,

$$
\partial^{*} a(n)=-\partial a(n-1) .
$$

We have then for a function $a$ defined on $\mathbb{N}_{\tau} \times \mathbb{N}_{\tau}$,

$$
\left(\partial_{n}-\partial_{n^{\prime}}^{*}\right) a\left(n, n^{\prime}\right)=a\left(n+1, n^{\prime}\right)-a\left(n, n^{\prime}-1\right) .
$$

We shall use below the following elementary formulas. For a function $a(n)$, denote $\tau_{k} a(n)=a(n-k)$ if $k \in \mathbb{Z}$. One has then

$$
\begin{aligned}
& \partial_{n}(a b)=\left(\partial_{n} a\right)\left(\tau_{-1} b\right)+a\left(\partial_{n} b\right), \\
& \partial_{n}^{*}(a b)=\left(\partial_{n}^{*} a\right) b+\left(\tau_{1} a\right)\left(\partial_{n}^{*} b\right), \\
& \partial_{n}(a b)=\left(\partial_{n} a\right) b+a\left(\partial_{n} b\right)+\left(\partial_{n} a\right)\left(\partial_{n} b\right), \\
& \partial_{n}^{*}(a b)=\left(\partial_{n}^{*} a\right) b+a\left(\partial_{n}^{*} b\right)+\left(\partial_{n}^{*} a\right)\left(\partial_{n}^{*} b\right) .
\end{aligned}
$$

Moreover, if we consider functions $a\left(n, n^{\prime}\right), b\left(n, n^{\prime}\right)$ defined on $\mathbb{N}_{\tau} \times \mathbb{N}_{\tau}$, and if $\tau_{k}^{1}, \tau_{k}^{2}$ are the translation operators relative to the first and second variable, respectively, we have

$$
\begin{aligned}
\left(\partial_{n}-\partial_{n^{\prime}}^{*}\right)(a b)=( & \left(\tau_{-1}^{1} a\right)\left(\left(\partial_{n}-\partial_{n^{\prime}}^{*}\right) b\right)+\left(\left(\partial_{n}-\partial_{n^{\prime}}^{*}\right) a\right)\left(\tau_{1}^{2} b\right), \\
\left(\partial_{n}-\partial_{n^{\prime}}^{*}\right)(a b)= & a\left(\left(\partial_{n}-\partial_{n^{\prime}}^{*}\right) b\right)+\left(\left(\partial_{n}-\partial_{n^{\prime}}^{*}\right) a\right) b+\left(\partial_{n} a\right)\left(\partial_{n} b\right)-\left(\partial_{n^{\prime}}^{*} a\right)\left(\partial_{n^{\prime}}^{*} b\right), \\
& \partial_{n}[a(n, n)]=\left(\left(\partial_{n}-\partial_{n^{\prime}}^{*}\right) a\right)(n, n+1), \\
& \partial_{n}^{*}[a(n, n)]=-\left(\left(\partial_{n}-\partial_{n^{\prime}}^{*}\right) a\right)(n-1, n) .
\end{aligned}
$$

Recall that a pseudo-differential operator $T$, of order 0 on $\mathbb{S}^{1}$, may be written when acting on a periodic function $u$ as

$$
T u(x)=\int_{\mathbb{S}^{1}} \sum_{n \in \mathbb{Z}} e^{i n(x-y)} a(x, n) u(y) d y,
$$

where $a$ is a smooth function on $\mathbb{S}^{1} \times \mathbb{Z}$, satisfying for any $\alpha, \beta \in \mathbb{N}$,

$$
\left|\partial_{x}^{\alpha} \partial_{n}^{\beta} a(x, n)\right| \leq C_{\alpha, \beta}(1+|n|)^{-\beta}
$$

(where $\partial_{x}$ means the usual derivative, and $\partial_{n}$ is defined by (1.2.3)). We set

$$
|a|_{P}=\sup _{0 \leq \alpha \leq P} \sup _{0 \leq \beta \leq P} \sup _{(x, n) \in \mathbb{S}^{1} \times \mathbb{Z}}(1+|n|)^{\beta}\left|\partial_{x}^{\alpha} \partial_{n}^{\beta} a(x, n)\right| .
$$

We may also use a local representation: Let $\chi \in C_{0}^{\infty}(\mathbb{R})$ be supported inside an interval of length strictly smaller than $2 \pi$. Take $\tilde{\chi} \in C_{0}^{\infty}(\mathbb{C}), \tilde{\chi} \equiv 1$ close to 0 , Supp $\tilde{\chi}$ small enough and set $\tilde{\chi}_{0}=1-\tilde{\chi}$. Define

$$
\begin{aligned}
\tilde{a}(x, \xi) & =\sum_{n=-\infty}^{+\infty} a(x, n) \Theta(x, \xi-n), \\
K(x, y) & =\sum_{n=-\infty}^{+\infty} e^{i n(x-y)} \tilde{\chi}_{0}\left(e^{i(x-y)}-1\right) a(x, n),
\end{aligned}
$$

with

$$
\Theta(x, \eta)=\int e^{-i(x-y) \eta} \tilde{\chi}\left(e^{i(x-y)}-1\right) \chi(y) d y
$$


Then we have if $\operatorname{Supp} u$ is contained in the domain where $\chi \equiv 1$,

$$
\begin{aligned}
& T u(x)=\frac{1}{2 \pi} \int e^{i x \xi} \tilde{a}(x, \xi) \hat{u}(\xi) d \xi+R u(x), \\
& R u(x)=\int K(x, y) u(y) d y .
\end{aligned}
$$

If we set $\tilde{\chi}_{k+1}(z)=z^{-1} \tilde{\chi}_{k}(z)$, we see that

$$
\begin{aligned}
K(x, y) & =\sum_{n}\left(e^{i(n+1)(x-y)}-e^{i n(x-y)}\right) \tilde{\chi}_{1}\left(e^{i(x-y)}-1\right) a(x, n) \\
& =\sum_{n} e^{i n(x-y)} \tilde{\chi}_{1}\left(e^{i(x-y)}-1\right) \partial_{n}^{*} a(x, n) \\
& =\sum_{n} e^{i n(x-y)} \tilde{\chi}_{k}\left(e^{i(x-y)}-1\right)\left(\partial_{n}^{*}\right)^{k} a(x, n) .
\end{aligned}
$$

This shows that $K$ is a smooth $2 \pi$-periodic function of $(x, y)$, whose derivatives up to order $N$ are bounded in $L^{\infty}$ in terms of the constants $C_{\alpha \beta}$ of (1.2.10) for $\alpha+\beta \leq N+2$. Moreover, if $x \in[-\pi, \pi]$ and Supp $\tilde{\chi}$ has been taken small enough, we see that

$$
\partial_{\eta} \Theta(x, \eta)=\int e^{-i(x-y) \eta}\left(e^{i(x-y)}-1\right) \tilde{\chi}^{1}(x, y) \chi(y) d y,
$$

where $\tilde{\chi}^{1}(x, y)=-i(x-y)\left(e^{i(x-y)}-1\right)^{-1} \tilde{\chi}\left(e^{i(x-y)}-1\right) \in C^{\infty}$ if $y \in \operatorname{Supp} \chi \Subset$ ]$-\pi, \pi\left[, x \in[-\pi, \pi]\right.$. Consequently $\partial_{\eta} \Theta(x, \eta)=\Theta^{1}(x, \eta-1)-\Theta^{1}(x, \eta)$, for a function $\Theta^{1}$, of the same form as $\Theta$, satisfying $\left|\partial_{x}^{\alpha} \Theta^{1}(x, \eta)\right| \leq C_{N}\langle\eta\rangle^{-N}$ for any $\alpha$, any $N$. We may thus write

$$
\partial_{\xi} \tilde{a}(x, \xi)=\sum_{n} a(x, n) \partial_{n}\left[\Theta^{1}(x, \xi-n)\right]=\sum_{n}\left(\partial_{n}^{*} a\right)(x, n) \Theta^{1}(x, \xi-n) .
$$

Computing in the same way higher order derivatives, we get that $\tilde{a}$ is a symbol on $[-\pi, \pi] \times \mathbb{R}$, whose semi-norms are controlled in terms of the corresponding semi-norms of $a$.

Our aim is to prove the following:

Theorem 1.2.1. There is $\tau \in \mathbb{N}^{*}$ and for any $n \geq \tau$, there is an orthonormal basis $\left(\varphi_{n}^{1}, \varphi_{n}^{2}\right)$ of $E_{n}$, satisfying the following property: there is $\nu \in \mathbb{R}_{+}$and for any $N, \alpha, \beta, \gamma \in \mathbb{N}$ there is a constant $C>0$, such that for any pseudo-differential operator of order 0 on $\mathbb{S}^{1}, T$, of symbol a, for any $n, n^{\prime} \in \mathbb{N}_{\tau}$, any $j, j^{\prime} \in\{1,2\}$, one has

$$
\left|\partial_{n}^{\alpha}\left(\partial_{n^{\prime}}^{*}\right)^{\beta}\left(\partial_{n}-\partial_{n^{\prime}}^{*}\right)^{\gamma}\left\langle\varphi_{n}^{j}, T \varphi_{n^{\prime}}^{j^{\prime}}\right\rangle\right| \leq C\left\langle n-n^{\prime}\right\rangle^{-N}\left(n+n^{\prime}\right)^{-\gamma}|a|_{\nu+N+\alpha+\beta+\gamma} .
$$

An Hilbertian basis $\left(\varphi_{n}^{j}\right)_{j, n}$ of $L^{2}\left(\mathbb{S}^{1}, \mathbb{R}\right)$, such that (1.2.14) is satisfied for $n, n^{\prime} \geq \tau$ large enough, will be called a nice basis.

Remark. The functions $\varphi_{n}^{1}, \varphi_{n}^{2}$ of the statement are not assumed to be eigenfunctions of $-\Delta+V$. Nevertheless, because of (1.2.2), they satisfy $\|(\sqrt{-\Delta+V}-$ $\omega(n))\left(\varphi_{n}^{j}\right) \|_{L^{2}}=O\left(n^{-\infty}\right)$.

Before starting the proof of the theorem, let us state a corollary. 
Corollary 1.2.2. Let $\left(\varphi_{n}^{j}\right)_{j, n}$ be a nice basis of $L^{2}\left(\mathbb{S}^{1}, \mathbb{R}\right)$. Let $T_{1}, T_{2}$ be two pseudodifferential operators of order 0 on $\mathbb{S}^{1}$. There is $\nu \in \mathbb{R}_{+}$, and for any $N, \alpha, \beta, \gamma \in \mathbb{N}$, there is $C>0$ such that for any $C^{\infty}$ function a on $\mathbb{S}^{1}$, one has

$$
\begin{aligned}
& \left|\partial_{n}^{\alpha}\left(\partial_{n^{\prime}}^{*}\right)^{\beta}\left(\partial_{n}-\partial_{n^{\prime}}^{*}\right)^{\gamma}\left\langle T_{1} \varphi_{n}^{j}, a(x) T_{2} \varphi_{n^{\prime}}^{j^{\prime}}\right\rangle\right| \\
& \leq C\left\langle n-n^{\prime}\right\rangle^{-N}\left(n+n^{\prime}\right)^{-\gamma} \sum_{k=0}^{\alpha+\beta+\gamma+N+\nu}\left\|\partial^{k} a\right\|_{L^{\infty}}
\end{aligned}
$$

for any $n, n^{\prime} \in \mathbb{N}^{*}$.

The corollary follows from (1.2.14) applied to $T=T_{1}^{*} a T_{2}$, which is a pseudodifferential operator of order 0 , whose symbol semi-norms $|\cdot|_{P}$ are controlled in terms of $\left\|\partial^{k} a\right\|_{L^{\infty}}$ for $k \leq P+\nu_{0}$, for a fixed $\nu_{0} \in \mathbb{N}$.

We shall first construct quasi-modes satisfying convenient properties.

Proposition 1.2.3. There exist for $n \geq \tau$ large enough, functions $\underline{U}_{n} \in$ $C^{\infty}([-\pi, \pi], \mathbb{C})$ satisfying the following properties:

(i) For any $n \in \mathbb{N}_{\tau}$, any $k \in \mathbb{N},\left\|\underline{U}_{n}\right\|_{L^{2}[-\pi, \pi]}=1$ and $\partial_{x}^{k} \underline{U}_{n}(\pi)-\partial_{x}^{k} \underline{U}_{n}(-\pi)=$ $O\left(n^{-\infty}\right), n \rightarrow+\infty$.

(ii) Let $T$ be a pseudo-differential operator of order 0 on $\mathbb{S}^{1}$. Denote by $U_{n}(x)$ the function on $\mathbb{R}$ obtained by $2 \pi$-periodization of $\underline{U}_{n}$. Consider $U_{n}$ as an element of $L^{2}\left(\mathbb{S}^{1}, \mathbb{C}\right)$, and define for $n, n^{\prime} \in \mathbb{N}_{\tau}$,

$$
I_{-}\left(n, n^{\prime}\right)=\left\langle T U_{n}, U_{n^{\prime}}\right\rangle, I_{+}\left(n, n^{\prime}\right)=\left\langle T U_{n}, \bar{U}_{n^{\prime}}\right\rangle .
$$

There is $\nu \in \mathbb{R}_{+}$, and for any $\alpha, \beta, \gamma, N \in \mathbb{N}$, a constant $C>0$ such that, for any operator $T$ as above, defined in terms of a symbol a by (1.2.9), one has

$$
\begin{gathered}
\left|\partial_{n}^{\alpha}\left(\partial_{n^{\prime}}^{*}\right)^{\beta}\left(\partial_{n}-\partial_{n^{\prime}}^{*}\right)^{\gamma} I_{-}\left(n, n^{\prime}\right)\right| \leq C\left\langle n-n^{\prime}\right\rangle^{-N}\left(n+n^{\prime}\right)^{-\gamma}|a|_{\nu+N+\alpha+\beta+\gamma} \\
\left|\partial_{n}^{\alpha}\left(\partial_{n^{\prime}}^{*}\right)^{\beta}\left(\partial_{n}-\partial_{n^{\prime}}^{*}\right)^{\gamma} I_{+}\left(n, n^{\prime}\right)\right| \leq C\left(n+n^{\prime}\right)^{-N-\gamma}|a|_{\nu+N+\alpha+\beta+\gamma}
\end{gathered}
$$

for any $n, n^{\prime} \in \mathbb{N}_{\tau}$ with $\left|n-n^{\prime}\right| \leq \frac{1}{2}\left(n+n^{\prime}\right)$.

(iii) There is a sequence $\left(h_{n}\right)_{n \in \mathbb{N}}$ of $\mathbb{R}_{+}^{*}$ such that $h_{n}^{-1}-\omega(n)=O\left(n^{-3}\right)$ and

$$
\left\|\left(-\Delta+V-h_{n}^{-2} \mathrm{Id}\right) U_{n}\right\|_{H^{-2}}=O\left(n^{-\infty}\right),\left\|U_{n}\right\|_{H^{1 / 2-\delta}} \leq C_{\delta} h_{n}^{-1}
$$

for any $n \geq \tau, \delta>0$.

We shall first construct $\underline{U}_{n}$ such that (i) and (iii) hold true.

Lemma 1.2.4. There are $\delta_{0}>0$ and smooth functions $(x, h) \rightarrow \theta(x, h),(x, h) \rightarrow$ $b(x, h)$ defined on $[-\pi, \pi] \times\left[0, \delta_{0}\right]$, real-valued, even in $h$, and a sequence $\left(h_{n}\right)_{n}$ of points of $] 0,1]$, with asymptotic expansion

$$
h_{n}=\frac{1}{n}-\frac{1}{4 \pi n^{3}} \int_{-\pi}^{\pi} V(x) d x+\sum_{k=2}^{N} \gamma_{k} n^{-2 k-1}+O\left(n^{-2 N-3}\right)
$$

for any $N \in \mathbb{N}$, such that the following properties hold true:

$$
\begin{gathered}
\frac{1}{h_{n}} \theta\left(\pi, h_{n}\right)-\frac{1}{h_{n}} \theta\left(-\pi, h_{n}\right)-2 \pi n=O\left(n^{-\infty}\right), \\
\theta^{\prime}(x, 0) \equiv 1,\left|\left(\partial_{x}^{\alpha} \partial_{h}^{\beta} \theta^{\prime}\right)(-\pi, h)-\left(\partial_{x}^{\alpha} \partial_{h}^{\beta} \theta^{\prime}\right)(\pi, h)\right|=O\left(h^{\infty}\right), \quad \forall \alpha, \beta \in \mathbb{N}, \\
\left|\partial_{x}^{\alpha} b(-\pi, h)-\partial_{x}^{\alpha} b(\pi, h)\right|=O\left(h^{\infty}\right)
\end{gathered}
$$


and such that if one sets

$$
\underline{U}_{n}(x)=e^{i \theta\left(x, h_{n}\right) / h_{n}} b\left(x, h_{n}\right),
$$

conditions (i) and (iii) of the statement of Proposition 1.2 .3 hold true.

Proof. We look for a formal series in $h, \Phi(x, h)$, with smooth coefficients in $x \in$ $[-\pi, \pi]$, such that $\operatorname{Im} \Phi(x, 0) \equiv 0$, and the semi-classical equation

$$
\left(-h^{2} \partial_{x}^{2}+h^{2} V(x)-1\right) e^{i \Phi(x, h) / h}=0
$$

is satisfied formally. We get, denoting by $\Phi^{\prime}, \Phi^{\prime \prime} x$-derivatives, the formal equation

$$
\Phi^{\prime}(x, h)^{2}-1-i h \Phi^{\prime \prime}(x, h)+h^{2} V(x) \equiv 0 .
$$

We look for a solution $\Phi^{\prime}(x, h)=\sum_{k=0}^{+\infty} h^{k} \Phi_{k}^{\prime}(x)$ with $\Phi_{0}^{\prime} \equiv 1, \Phi_{2 k}^{\prime}$ real, and $\Phi_{2 k+1}^{\prime}$ purely imaginary. Identifying powers of $h$ we get for $k \geq 1$,

$$
\Phi_{k}^{\prime}(x)=-\frac{1}{2} V(x) \delta_{k 2}-\frac{1}{2} \sum_{\ell=1}^{k-1} \Phi_{\ell}^{\prime}(x) \Phi_{k-\ell}^{\prime}(x)+\frac{i}{2} \Phi_{k-1}^{\prime \prime}(x),
$$

whence

$$
\Phi_{1}^{\prime}(x) \equiv 0, \Phi_{2}^{\prime}(x)=-\frac{1}{2} V(x), \Phi_{k}^{\prime}(x) 2 \pi \text {-periodic for any } k .
$$

Taking the imaginary part of (1.2.25), we get

$$
\operatorname{Re} \Phi^{\prime}(x, h) \operatorname{Im} \Phi^{\prime}(x, h)=\frac{h}{2} \operatorname{Re} \Phi^{\prime \prime}(x, h) .
$$

We choose for the equation on $\operatorname{Im} \Phi$ the solution

$$
\operatorname{Im} \Phi(x, h)=\frac{h}{2} \log \left[\operatorname{Re} \Phi^{\prime}(x, h)\right]
$$

where the right hand side is well defined since $\operatorname{Re} \Phi^{\prime}(x, 0) \equiv 1$. We thus see that $\operatorname{Im} \Phi(x, h)$ is $2 \pi$-periodic in $x$ and odd in $h$. We may write using (1.2.26),

$$
\Phi(\pi, h)-\Phi(-\pi, h)=\int_{-\pi}^{\pi} \operatorname{Re} \Phi^{\prime}(x, h) d x=2 \pi-\frac{h^{2}}{2} \int_{-\pi}^{\pi} V(x) d x+\sum_{k=2}^{+\infty} A_{k} h^{2 k}
$$

for some real constants $A_{k}$. Then $e^{i \Phi(x, h) / h}$ will be $2 \pi$-periodic if and only if there is $n \in \mathbb{N}$ with $\Phi(\pi, h)-\Phi(-\pi, h)=2 \pi n h$. By (1.2.28), the $h$-solutions of this equation for $n$ large enough form a sequence $\left(h_{n}\right)_{n}$ of $\mathbb{R}_{+}^{*}$, converging to zero, and having asymptotic expansion

$$
h_{n}=\frac{1}{n}-\frac{1}{4 \pi n^{3}} \int_{-\pi}^{\pi} V(x) d x+\cdots .
$$

Comparison with (1.2.1) shows that $h_{n}^{-1}-\omega(n)=O\left(n^{-3}\right)$.

We denote by $\theta(x, h)$ (resp. $\tilde{b}(x, h))$ a smooth function of $(x, h)$ on $[-\pi, \pi] \times\left[0, \delta_{0}\right]$, even in $h$, whose difference with $\operatorname{Re} \Phi(x, h)$ (resp. $\left.e^{-\operatorname{Im} \Phi(x, h) / h}\right)$ is tangent to 0 at infinite order, as well as its derivatives, when $h \rightarrow 0$, uniformly in $x \in[-\pi, \pi]$. Since $\operatorname{Im} \Phi(x, h)$ and $\operatorname{Re} \Phi^{\prime}(x, h)$ are $2 \pi$-periodic for any $h,(1.2 .22)$ with $b$ replaced by $\tilde{b}$ holds true. Moreover, by (1.2.26), (1.2.27), $\tilde{b}(x, h)=1+O\left(h^{2}\right)$ uniformly in $x \in$ $[-\pi, \pi]$, so $\|\tilde{b}(\cdot, h)\|_{L^{2}([-\pi, \pi])}=\sqrt{2 \pi}+O\left(h^{2}\right)$. If we set $b(x, h)=\tilde{b}(x, h) /\|\tilde{b}(\cdot, h)\|_{L^{2}}$, we thus obtain a function satisfying the last relation (1.2.22). The equality (1.2.21) follows from the definition of $h_{n}$. Define now $\underline{U}_{n}(x, h)=e^{i \theta\left(x, h_{n}\right) / h_{n}} b\left(x, h_{n}\right)$. It 
obeys the properties of (i) of Proposition 1.2.3. Moreover, by (1.2.24), we have the equality $\left(-\Delta+V-h_{n}^{-2}\right) \underline{U}_{n}=O\left(h_{n}^{\infty}\right)$ on $[-\pi, \pi]$. If $U_{n}$ is the $2 \pi$-periodization of $\underline{U}_{n}$, then $U_{n}$ is in $L^{2}\left(\mathbb{S}^{1}, \mathbb{C}\right)$, but not in $C^{\infty}\left(\mathbb{S}^{1}\right)$, since it has, as well as its derivatives, jumps of magnitude $O\left(h_{n}^{\infty}\right)$ at $\pi \bmod 2 \pi$. Consequently, $\left(-\Delta+V-h_{n}^{-2}\right) U_{n}=$ $\alpha_{n} \delta_{\pi}+\beta_{n} \delta_{\pi}^{\prime}+g_{n}(x)$ where $\alpha_{n}, \beta_{n}=O\left(h_{n}^{\infty}\right), g_{n}$ is $C^{\infty}$ on $[-\pi, \pi]$ and $O\left(h_{n}^{\infty}\right)$. This gives the first inequality in (1.2.19). The second one follows from the fact that by (1.2.23), $\nabla U_{n}=\alpha_{n} \delta_{\pi}+r_{n}$ with $\alpha_{n}=O\left(h_{n}^{\infty}\right),\left\|r_{n}\right\|_{L^{2}}=O\left(h_{n}^{-1}\right)$, whence $\left\|\nabla U_{n}\right\|_{H^{-1 / 2-\delta}}=O\left(h_{n}^{-1}\right)$ for any $\delta>0$.

We now want to express the quantities (1.2.16) in terms of Fourier integrals. Recall that we consider a pseudo-differential operator $T$ of order 0 , expressed in terms of its symbol $a$ by (1.2.9).

Lemma 1.2.5. There is $\nu \in \mathbb{R}_{+}$, a finite set of indices $\mathcal{J}$, and for any $N \in \mathbb{N}$, functions $r_{N}^{ \pm}: \mathbb{N}_{\tau} \times \mathbb{N}_{\tau} \rightarrow \mathbb{C}$ satisfying for any $\alpha, \beta, \gamma$,

$$
\left|\partial_{n}^{\alpha}\left(\partial_{n^{\prime}}^{*}\right)^{\beta}\left(\partial_{n}-\partial_{n^{\prime}}^{*}\right)^{\gamma} r_{N}\left(n, n^{\prime}\right)\right| \leq C_{\alpha \beta \gamma N}\left(n+n^{\prime}\right)^{-N-\gamma}|a|_{N+\alpha+\beta+\gamma+\nu}
$$

and a family of functions $A_{N}^{j, \pm}: \mathbb{R}^{3} \times \mathbb{R}_{+}^{2} \rightarrow \mathbb{C}$,

$$
\left(x, y, \xi, \omega, \omega^{\prime}\right) \rightarrow A_{N}^{j, \pm}\left(x, y, \xi, \omega, \omega^{\prime}\right),
$$

compactly supported with respect to $(x, y, \xi)$, smooth in $\left(\omega, \omega^{\prime}\right)$, satisfying for $\left|\omega-\omega^{\prime}\right|$ $\leq \frac{1}{2}\left(\omega+\omega^{\prime}\right)$ estimates of the type

$$
\begin{aligned}
& \left|\partial_{\omega}^{\alpha} \partial_{\omega^{\prime}}^{\beta}\left(\partial_{\omega}+\partial_{\omega^{\prime}}\right)^{\gamma} A_{N}^{j, \pm}\left(x, y, \xi, \omega, \omega^{\prime}\right)\right| \\
& \quad \leq C_{\alpha \beta \gamma N N^{\prime}}|a|_{N+N^{\prime}+\alpha+\beta+\gamma}(1+|x-y| \omega)^{-N^{\prime}}\left\langle\omega \pm \omega^{\prime}\right\rangle^{-N}\left(\omega+\omega^{\prime}\right)^{-\gamma}
\end{aligned}
$$

for any $\alpha, \beta, \gamma, N^{\prime}$, such that if

$$
J_{N}^{j, \pm}\left(\omega, \omega^{\prime}\right)=\omega \int_{\mathbb{R}^{3}} e^{i\left[\omega(x-y) \xi+\omega \theta\left(y, \frac{1}{\omega}\right) \pm \omega^{\prime} \theta\left(x, \frac{1}{\omega^{\prime}}\right)\right]} A_{N}^{j, \pm}\left(x, y, \xi, \omega, \omega^{\prime}\right) d x d y d \xi,
$$

one has for $\left|n-n^{\prime}\right| \leq \frac{1}{2}\left(n+n^{\prime}\right)$,

$$
I_{ \pm}\left(n, n^{\prime}\right)=\sum_{j \in \mathcal{J}} J_{N}^{j, \pm}\left(h_{n}^{-1}, h_{n^{\prime}}^{-1}\right)+r_{N}^{ \pm}\left(n, n^{\prime}\right) .
$$

Proof. If we use (1.2.9), (1.2.13) and a partition of unity in $y$, we may write $T v$ as the sum of $R v$ (where $R$ is a smoothing operator whose contribution will be discussed at the end of the proof) and of a finite sum of integrals of the form

$$
\int_{\mathbb{R}^{2}} e^{i(x-y) \xi} \tilde{a}(x, y, \xi) v(y) d y d \xi
$$

where $v$ is the $2 \pi$-periodic extension of $v \in L^{2}\left(\mathbb{S}^{1}, \mathbb{R}\right)$, where $\tilde{a}$ is $C^{\infty}$ in $(x, y, \xi)$, compactly supported in $(x, y)$, and satisfies

$$
\left|\partial_{x}^{\alpha} \partial_{y}^{\beta} \partial_{\xi}^{\gamma} \tilde{a}(x, y, \xi)\right| \leq C_{\alpha \beta \gamma}(1+|\xi|)^{-\gamma}
$$

with constants $C_{\alpha \beta \gamma}$ controlled in terms of $|a|_{\alpha+\beta+\gamma}$. Let $\chi_{1} \in C^{\infty}(\mathbb{R}), \chi_{1} \equiv 0$ on $[-1,1], \chi_{1} \equiv 1$ outside $[-2,2]$, and define

$$
T^{n} v(x)=\int e^{i(x-y) \xi} \tilde{a}(x, y, \xi) \chi_{1}\left(n^{-2} \xi\right) v(y) d y d \xi .
$$

Let us take $v=U_{n}$, a $2 \pi$-periodic extension of the function $\underline{U}_{n}$ defined on $[-\pi, \pi]$ by (1.2.23). Recall that $U_{n}$ is smooth outside $\pi+2 \pi \mathbb{Z}$, and that at all points of $\pi+2 \pi \mathbb{Z}$, 
$U_{n}$ as well as its derivatives has a jump of magnitude $O\left(n^{-\infty}\right)$. Consequently, when we perform in (1.2.35) one integration by parts in $y$, we get

$$
T^{n} v(x)=\int e^{i(x-y) \xi} \mathbf{1}_{\{y-\pi \notin 2 \pi \mathbb{Z}\}} \partial_{y}\left[\tilde{a}(x, y, \xi) \frac{\chi_{1}\left(n^{-2} \xi\right)}{i \xi} U_{n}(y)\right] d y d \xi+T_{1}^{n} w,
$$

where $T_{1}^{n}$ is an operator of order -1 , acting on a distribution $w$ which is a finite sum of Dirac masses with coefficients $O\left(n^{-\infty}\right)$. In particular, $\left\|T_{1}^{n} w\right\|_{L^{2}}=O\left(n^{-\infty}\right)$. If we perform more integrations by parts, we may write, remarking that each integration gains $n^{-2}$ and loses one $\partial_{y}$ derivative,

$$
\left\|T^{n} v\right\|_{L^{2}} \leq C_{N}|a|_{N+\nu} n^{-2 N}\left\|\underline{U}_{n}\right\|_{H^{N}([-\pi, \pi])}
$$

for a fixed $\nu \in \mathbb{R}_{+}$. Since by (1.2.23), $\left\|\underline{U}_{n}\right\|_{H^{N}}=O\left(n^{N}\right)$, we see that the contribution of $T^{n}$ to $I_{ \pm}\left(n, n^{\prime}\right)$ contributes to the last term in (1.2.32). This shows that we may, from now on, replace $T$ by the operator $T_{n}$ defined by

$$
T_{n} v(x)=\int e^{i(x-y) \xi} \tilde{a}(x, y, \xi) \chi\left(n^{-2} \xi\right) v(y) d y d \xi
$$

where $\chi=1-\chi_{1}$, and study instead of $I_{-}\left(n, n^{\prime}\right)$ (resp. $I_{+}\left(n, n^{\prime}\right)$ ) the quantity $\left\langle T_{n} U_{n}, U_{n^{\prime}}\right\rangle$ (resp. $\left\langle T_{n} U_{n}, \bar{U}_{n^{\prime}}\right\rangle$ ), i.e. respectively

$$
\int_{\mathbb{R}^{3}} e^{i(x-y) \xi+\frac{i}{h_{n}} \theta\left(y, h_{n}\right) \mp \frac{i}{h_{n^{\prime}}} \theta\left(x, h_{n^{\prime}}\right)} \tilde{a}(x, y, \xi) \chi\left(n^{-2} \xi\right) b\left(y, h_{n}\right) b^{\mp}\left(x, h_{n^{\prime}}\right) d x d y d \xi
$$

with $b^{+} \equiv b, b^{-} \equiv \bar{b}$. If we make in (1.2.36) integrations by parts in $x$ or $y$, because $\theta$ or $b$ have jumps at $\pi+2 \pi \mathbb{Z}$, we shall get boundary terms. But (1.2.21), (1.2.22), and the fact that $\xi$ is localized in a region where $|\xi| \leq C n^{2}$, show us that these contributions will give rise to admissible remainders of type (1.2.29). Consequently, we may argue as if $\theta$ and $b$ were $C^{\infty} 2 \pi$-periodic functions. Remark that by the first relation (1.2.22), we shall have $\left|\xi-\frac{1}{h_{n}} \theta^{\prime}\left(y, h_{n}\right)\right| \geq \frac{c}{h_{n}}$ if $h_{n}$ is small enough, and either $|\xi| \geq A h_{n}^{-1}$ or $|\xi| \leq A^{-1} h_{n}^{-1}$ for a large enough constant $A>0$. Consequently, using $y$-integrations by parts, we see that up to admissible remainders of type (1.2.29), we may in (1.2.36) replace the cut-off $\chi\left(n^{-2} \xi\right)$ by $\varphi\left(h_{n} \xi\right)$ with $\varphi \in C_{0}^{\infty}(\mathbb{R}-\{0\})$. We are thus reduced to

$$
\frac{1}{h_{n}} \int e^{i\left[\frac{1}{h_{n}}(x-y) \xi+\frac{1}{h_{n}} \theta\left(y, h_{n}\right) \mp \frac{1}{h_{n^{\prime}}} \theta\left(x, h_{n^{\prime}}\right)\right]} \tilde{a}\left(x, y, \frac{\xi}{h_{n}}\right) \varphi(\xi) b\left(y, h_{n}\right) b^{\mp}\left(x, h_{n^{\prime}}\right) d x d y d \xi .
$$

Define the vector field

$$
\begin{aligned}
L_{\mp}\left(x, y, \omega, \omega^{\prime}, \partial_{x}\right. & \left.+\partial_{y}\right)=\left(1+\left(\omega \theta^{\prime}\left(y, \frac{1}{\omega}\right) \mp \omega^{\prime} \theta^{\prime}\left(x, \frac{1}{\omega^{\prime}}\right)\right)^{2}\right)^{-1} \\
& \times\left[1+\left(\omega \theta^{\prime}\left(y, \frac{1}{\omega}\right) \mp \omega^{\prime} \theta^{\prime}\left(x, \frac{1}{\omega^{\prime}}\right)\right)\left(\partial_{x}+\partial_{y}\right)\right] .
\end{aligned}
$$

Since $\theta^{\prime}(x, h)$ is even in $h$, and $\theta^{\prime}(x, 0) \equiv 1$, we may write

$$
\omega \theta^{\prime}\left(y, \frac{1}{\omega}\right) \mp \omega^{\prime} \theta^{\prime}\left(x, \frac{1}{\omega^{\prime}}\right)=\omega \mp \omega^{\prime}+\sigma(y, \omega) \mp \sigma\left(x, \omega^{\prime}\right),
$$

where $\sigma(y, \omega)$ satisfies for any $\alpha, \gamma \in \mathbb{N}$ (using (1.2.22) )

$$
\begin{aligned}
& \left|\partial_{y}^{\alpha} \partial_{\omega}^{\gamma} \sigma(y, \omega)\right| \leq C_{\alpha \gamma}(1+\omega)^{-1-\gamma} \quad \forall y \in \mathbb{R}-\{\pi+2 \pi \mathbb{Z}\}, \forall \omega \in \mathbb{R}_{+}, \\
& {\left[\partial_{y}^{\alpha} \partial_{\omega}^{\gamma} \sigma\right]=O\left(\omega^{-\infty}\right),}
\end{aligned}
$$


denoting by [.] the jump at $\pi+2 \pi \mathbb{Z}$. Consequently, the coefficients $c\left(x, y, \omega, \omega^{\prime}\right)$ of $L_{\mp}$ satisfy for $x, y$ outside $\pi+2 \pi \mathbb{Z}$,

$$
\left|\partial_{x}^{\delta} \partial_{y}^{\delta^{\prime}} \partial_{\omega}^{\alpha} \partial_{\omega^{\prime}}^{\beta}\left(\partial_{\omega}+\partial_{\omega^{\prime}}\right)^{\gamma} c\left(x, y, \omega, \omega^{\prime}\right)\right| \leq C\left(1+\omega+\omega^{\prime}\right)^{-\gamma}\left\langle\omega \mp \omega^{\prime}\right\rangle^{-1}
$$

when $\left|\omega-\omega^{\prime}\right| \leq \frac{1}{2}\left(\omega+\omega^{\prime}\right)$, with jump conditions

$$
\left[\partial_{x}^{\delta} \partial_{y}^{\delta^{\prime}} \partial_{\omega}^{\alpha} \partial_{\omega^{\prime}}^{\beta} c\right]=O\left(\left(\omega+\omega^{\prime}\right)^{-\infty}\right) .
$$

We make in (1.2.37) integrations by parts using the vector field (1.2.38). Again, because of (1.2.41) and (1.2.21), (1.2.22), boundary terms coming from the jumps give rise to remainders of type (1.2.29), and up to such perturbations, we may rewrite (1.2.37) as

$$
\begin{aligned}
\frac{1}{h_{n}} \int e^{i\left[\frac{1}{h_{n}}(x-y) \xi+\right.} & \left.\frac{1}{h_{n}} \theta\left(y, h_{n}\right) \mp \frac{1}{h_{n^{\prime}}} \theta\left(x, h_{n^{\prime}}\right)\right] \\
& \times\left({ }^{t} L_{\mp}\right)^{N}\left[\tilde{a}\left(x, y, \frac{\xi}{h_{n}}\right) \varphi(\xi) b\left(y, h_{n}\right) b^{\mp}\left(x, h_{n^{\prime}}\right)\right] d x d y d \xi .
\end{aligned}
$$

If $L_{0}\left(x-y, \omega, \partial_{\xi}\right)=\left(1+\omega^{2}(x-y)^{2}\right)^{-1}\left(1+\omega(x-y) \cdot \partial_{\xi}\right)$, the coefficients of $L_{0}$ satisfy the estimates

$$
\left|\partial_{\omega}^{\alpha} c(x-y, \omega)\right| \leq C_{\alpha}(1+\omega|x-y|)^{-1} \omega^{-\alpha} .
$$

Integrating by parts using $L_{0}$, we obtain that (1.2.42) may be written as $J_{N}^{\mp}\left(h_{n}^{-1}, h_{n^{\prime}}^{-1}\right)$ with

$$
J_{N}^{\mp}\left(\omega, \omega^{\prime}\right)=\omega \int e^{i\left[\omega(x-y) \xi+\omega \theta\left(y, \frac{1}{\omega}\right) \mp \omega^{\prime} \theta\left(x, \frac{1}{\omega^{\prime}}\right)\right]} A_{N}^{\mp}\left(x, y, \xi, \omega, \omega^{\prime}\right) d x d y d \xi
$$

with

$$
A_{N}^{\mp}=\left({ }^{t} L_{0}\right)^{N^{\prime}}\left({ }^{t} L_{\mp}\right)^{N}\left[\tilde{a}(x, y, \omega \xi) \varphi(\xi) b\left(y, \frac{1}{\omega}\right) b^{\mp}\left(x, \frac{1}{\omega^{\prime}}\right)\right] .
$$

By (1.2.40), (1.2.43), and (1.2.34), $A_{N}^{\mp}$ satisfies (1.2.30). Finally, the contributions $\left\langle R U_{n}, U_{n^{\prime}}\right\rangle,\left\langle R U_{n}, \bar{U}_{n^{\prime}}\right\rangle$ of the smoothing operator in (1.2.13) to $I_{+}, I_{-}$contribute to $r_{N}^{ \pm}$in (1.2.32), using (1.2.23) and integrations by parts. This proves the lemma.

Proof of Proposition 1.2.3. By Lemma 1.2.4, conditions (i) and (iii) of the statement of the proposition hold true. Let us prove (1.2.18). Since $h_{n}^{-1}=n+O(1 / n)$, if we plug (1.2.30) with $\alpha=\beta=\gamma=0$ inside (1.2.31) and integrate in $y$, we get from (1.2.32) that there is a fixed $\nu \in \mathbb{R}_{+}$such that for any $N,\left|I_{+}\left(n, n^{\prime}\right)\right| \leq$ $C_{N}\left(n+n^{\prime}\right)^{-N}|a|_{N+\nu}$ when $\left|n-n^{\prime}\right| \leq \frac{1}{2}\left(n+n^{\prime}\right)$. This implies (1.2.18).

To show (1.2.17), let us prove first that for $\left|\omega-\omega^{\prime}\right| \leq \frac{1}{2}\left(\omega+\omega^{\prime}\right)$,

$$
\left|\partial_{\omega}^{\alpha} \partial_{\omega^{\prime}}^{\beta}\left(\partial_{\omega}+\partial_{\omega^{\prime}}\right)^{\gamma} J_{N}^{j,-}\left(\omega, \omega^{\prime}\right)\right| \leq C\left\langle\omega-\omega^{\prime}\right\rangle^{-N}\left(\omega+\omega^{\prime}\right)^{-\gamma}|a|_{\alpha+\beta+\gamma+N+2}
$$

Remark first that if we make $\partial_{\omega}+\partial_{\omega^{\prime}}$ act on the phase of $J_{N}^{j,-}$, we get either a contribution which is $O\left(\omega^{-1}+\omega^{\prime-1}\right)$, or a quantity such as $i(x-y) \xi$ or $i\left[\theta\left(y, \frac{1}{\omega}\right)-\theta\left(x, \frac{1}{\omega^{\prime}}\right)\right]$, in which, modulo an $O\left(\omega^{-1}+\omega^{\prime-1}\right)$ term, we may factor out $x-y$. The decay given by the $N^{\prime}$ exponent in (1.2.30) allows one to transform such a term in a gain of one negative power of $\omega$. Consequently, (1.2.44) follows from $y$-integrations of estimates (1.2.30). We have then to show that (1.2.44) implies that

$$
\partial_{n}^{\alpha}\left(\partial_{n^{\prime}}^{*}\right)^{\beta}\left(\partial_{n}-\partial_{n^{\prime}}^{*}\right)^{\gamma} J_{N}^{j,-}\left(\frac{1}{h_{n}}, \frac{1}{h_{n^{\prime}}}\right)
$$


is estimated by the right hand side of (1.2.17). Call $\tilde{\omega}(\lambda)$ a symbol of order 1 defined on $\mathbb{R}_{+}$, such that according to (1.2.20), $h_{n}^{-1}-\tilde{\omega}(n)=O\left(n^{-\infty}\right)$. Up to terms satisfying estimates of type (1.2.29) we may, instead of (1.2.45), bound

$$
\partial_{n}^{\alpha}\left(\partial_{n^{\prime}}^{*}\right)^{\beta}\left(\partial_{n}-\partial_{n^{\prime}}^{*}\right)^{\gamma} J_{N}^{j,-}\left(\tilde{\omega}(n), \tilde{\omega}\left(n^{\prime}\right)\right) .
$$

We use induction on $\alpha+\beta+\gamma$ : set for $t \in[0,1], \Omega(n, t)=t \tilde{\omega}(n+1)+(1-t) \tilde{\omega}(n)$ so that

$$
\begin{aligned}
\left(\partial_{n}-\right. & \left.\partial_{n^{\prime}}^{*}\right) J_{N}^{j,-}\left(\tilde{\omega}(n), \tilde{\omega}\left(n^{\prime}\right)\right)=J_{N}^{j,-}\left(\tilde{\omega}(n+1), \tilde{\omega}\left(n^{\prime}\right)\right)-J_{N}^{j,-}\left(\tilde{\omega}(n), \tilde{\omega}\left(n^{\prime}-1\right)\right) \\
= & \int_{0}^{1}\left(\partial_{\omega} J_{N}^{j,-}\right)\left(\Omega(n, t), \Omega\left(n^{\prime}-1, t\right)\right) d t(\tilde{\omega}(n+1)-\tilde{\omega}(n)) \\
& +\int_{0}^{1}\left(\partial_{\omega^{\prime}} J_{N}^{j,-}\right)\left(\Omega(n, t), \Omega\left(n^{\prime}-1, t\right)\right) d t\left(\tilde{\omega}\left(n^{\prime}\right)-\tilde{\omega}\left(n^{\prime}-1\right)\right) .
\end{aligned}
$$

Since $\tilde{\omega}(\lambda)-\lambda$ is a symbol of order -1 , we may write this as

$$
\begin{gathered}
\int_{0}^{1}\left(\partial_{\omega}+\partial_{\omega^{\prime}}\right) J_{N}^{j,-}\left(\Omega(n, t), \Omega\left(n^{\prime}-1, t\right)\right) d t+\int_{0}^{1} \partial_{\omega} J_{N}^{j,-}\left(\Omega(n, t), \Omega\left(n^{\prime}-1, t\right)\right) d t \tilde{\omega}_{-2}(n) \\
+\int_{0}^{1} \partial_{\omega^{\prime}} J_{N}^{j,-}\left(\Omega(n, t), \Omega\left(n^{\prime}-1, t\right)\right) d t \tilde{\omega}_{-2}\left(n^{\prime}-1\right)
\end{gathered}
$$

for a new symbol $\tilde{\omega}_{-2}(\lambda)$ of order -2 . This shows that we gained one (actually two) negative powers of $n+n^{\prime}$ in the last two integrals, when $\left|n-n^{\prime}\right| \leq \frac{1}{2}\left(n+n^{\prime}\right)$, and also one such power in the first integral, because of (1.2.44). Moreover, $\Omega(n, t)$ satisfies the same assumptions as $\tilde{\omega}(n)$, which allows one to proceed with the induction. This concludes the proof of the proposition.

Lemma 1.2.6. Let $\lambda \rightarrow \omega(\lambda)$ be the symbol defined after (1.2.1). Then

$$
\frac{1}{h_{n}}-\omega(n)=O\left(n^{-\infty}\right) .
$$

Moreover, for $n$ large enough, there is a real-valued orthonormal basis $\left(\varphi_{n}^{1}, \varphi_{n}^{2}\right)$ of the space $E_{n}$ such that

$$
\left\|\varphi_{n}^{1}-\frac{U_{n}+\bar{U}_{n}}{\sqrt{2}}\right\|_{L^{2}}=O\left(n^{-\infty}\right), \quad\left\|\varphi_{n}^{2}-\frac{U_{n}-\bar{U}_{n}}{i \sqrt{2}}\right\|_{L^{2}}=O\left(n^{-\infty}\right) .
$$

Proof. We denote by $F_{n}$ the span of $\left(U_{n}^{1}, U_{n}^{2}\right)$ in $L^{2}\left(\mathbb{S}^{1}, \mathbb{R}\right)$, where $U_{n}^{1}=\frac{U_{n}+\bar{U}_{n}}{\sqrt{2}}, U_{n}^{2}=$ $\frac{U_{n}-\bar{U}_{n}}{i \sqrt{2}}$. Then for $v \in F_{n}$, if $P=-\frac{d^{2}}{d x^{2}}+V(x)$, we have by (iii) of Proposition 1.2.3.

$$
\left\|\left(P-h_{n}^{-2}\right) v\right\|_{H^{-2}}=O\left(n^{-\infty}\right)
$$

uniformly for $v$ staying in the unit ball of $F_{n}$. In the same way, since $E_{n}$ is the range of the spectral projector $\Pi_{n}$ associated to the couple of eigenvalues $\left(\omega_{n}^{-}\right)^{2} \leq\left(\omega_{n}^{+}\right)^{2}$, we have by (1.2.2),

$$
\left\|\left(P-\omega(n)^{2}\right) v\right\|_{H^{-2}}=O\left(n^{-\infty}\right)
$$

uniformly for $v$ in the unit ball of $E_{n}$ (actually, the above relation holds true even for the $L^{2}$ norm). We shall denote by $E_{n}^{\perp}$ the orthogonal complement of $E_{n}$ in $H^{-2}$, by $\Pi_{n}^{\perp}: H^{-2} \rightarrow E_{n}^{\perp}$ the orthogonal projection, and shall also use the notation $\Pi_{n}$ for the orthogonal projector from $H^{-2}$ to $E_{n}$. We set $Q_{n}=\Pi_{n}^{\perp}\left(P-\omega(n)^{2} \mathrm{Id}\right) \Pi_{n}^{\perp}$ considered as a bounded operator from $E_{n}^{\perp} \cap L^{2}$ to $E_{n}^{\perp}$. Since the eigenvalues of $P$ different from $\left(\omega_{n}^{+}\right)^{2}$ and $\left(\omega_{n}^{-}\right)^{2}$ lie at a distance from $\omega(n)^{2}$ bounded from below by 
a fixed constant, $Q_{n}$ is invertible, with inverse $Q_{n}^{-1}: E_{n}^{\perp} \rightarrow E_{n}^{\perp} \cap L^{2}$ whose norm in $\mathcal{L}\left(H^{-2}, L^{2}\right)$ depends on $n$, but with $\left\|Q_{n}^{-1}\right\|_{\mathcal{L}\left(H^{-2}, H^{-2}\right)}$ uniformly bounded. Since we have seen in Proposition 1.2.3 that $\omega(n)-h_{n}^{-1}=O\left(n^{-3}\right)$, the operator

$$
\text { Id }-Q_{n}^{-1}\left(h_{n}^{-2}-\omega(n)^{2}\right)
$$

will be invertible, as an operator from $E_{n}^{\perp}$ to $E_{n}^{\perp}$ endowed with the $H^{-2}$ norm, for large enough $n$. If $v$ is in the unit ball of $L^{2}$, we have

(1.2.51) $Q_{n} v=\Pi_{n}^{\perp}\left(P-\omega(n)^{2} \mathrm{Id}\right) \Pi_{n}^{\perp} v=\Pi_{n}^{\perp}\left(P-\omega(n)^{2} \mathrm{Id}\right) v-\Pi_{n}^{\perp}\left(P-\omega(n)^{2} \mathrm{Id}\right) \Pi_{n} v$.

By (1.2.2), the last term has $L^{2}$ (or $H^{-2}$ ) norm $O\left(n^{-\infty}\right)$. If we assume moreover that $v \in F_{n}$, and write

$$
\left(P-\omega(n)^{2} \mathrm{Id}\right) v=\left(h_{n}^{-2}-\omega(n)^{2}\right) v+\left(P-h_{n}^{-2}\right) v,
$$

the last term has $H^{-2}$ norm $O\left(n^{-\infty}\right)$ by (1.2.48). We deduce from this equality and (1.2.51),

$$
\left(Q_{n}-\left(h_{n}^{-2}-\omega(n)^{2}\right) \mathrm{Id}\right) \Pi_{n}^{\perp} v=r_{n}
$$

with $r_{n} \in E_{n}^{\perp},\left\|r_{n}\right\|_{H^{-2}}=O\left(n^{-\infty}\right)$. We deduce from the invertibility of $Q_{n}$ and of (1.2.50) for large enough $n$ that

$$
\left\|\Pi_{n}^{\perp} v\right\|_{H^{-2}}=O\left(n^{-\infty}\right) .
$$

We set for $n$ large enough $\psi_{n}^{1}=\Pi_{n} U_{n}^{1}, \psi_{n}^{2}=\Pi_{n} U_{n}^{2}$. The above equality implies

$$
\left\|\psi_{n}^{1}-U_{n}^{1}\right\|_{H^{-2}}=O\left(n^{-\infty}\right), \quad\left\|\psi_{n}^{2}-U_{n}^{2}\right\|_{H^{-2}}=O\left(n^{-\infty}\right) .
$$

Moreover, since $\psi_{n}^{j}$ is in the range of $\Pi_{n},\left\|\psi_{n}^{j}\right\|_{H^{\frac{1}{2}-\delta}} \leq C h_{n}^{-\frac{1}{2}+\delta}$ for any $\delta>0$, so that using (1.2.19), $\left\|\psi_{n}^{j}-U_{n}^{j}\right\|_{H^{\frac{1}{2}-\delta}} \leq C h_{n}^{-1}$. Interpolating with (1.2.53), we get

$$
\left\|\psi_{n}^{j}-U_{n}^{j}\right\|_{L^{2}}=O\left(n^{-\infty}\right), \quad j=1,2 .
$$

Since $\left\|U_{n}\right\|_{L^{2}}=1$, and $\left\langle U_{n}, \bar{U}_{n}\right\rangle=O\left(n^{-\infty}\right)$ by (1.2.16) and (1.2.18), we deduce from (1.2.54) and the definition of $U_{n}^{1}, U_{n}^{2}$ that

$$
\left\langle\psi_{n}^{1}, \psi_{n}^{2}\right\rangle=O\left(n^{-\infty}\right), \quad\left\|\psi_{n}^{j}\right\|_{L^{2}}^{2}-1=O\left(n^{-\infty}\right) .
$$

We define now $\left(\varphi_{n}^{1}, \varphi_{n}^{2}\right)$ as a Gram-Schmidt orthonormalization of $\left(\psi_{n}^{1}, \psi_{n}^{2}\right)$. Then (1.2.47) follows from (1.2.54), (1.2.55). To show (1.2.46), we take $v \in F_{n}$ of norm 1. We write

$$
\left(\omega(n)^{2}-h_{n}^{-2}\right) \Pi_{n} v=-\left(P-\omega(n)^{2}\right) \Pi_{n} v+\left(P-h_{n}^{-2}\right) v-P \Pi_{n}^{\perp} v+h_{n}^{-2} \Pi_{n}^{\perp} v .
$$

By (1.2.48), (1.2.49), the $H^{-2}$ norm of the first two terms on the right hand side is $O\left(n^{-\infty}\right)$. By (1.2.52), the $H^{-4}$ norm of the last two terms is $O\left(n^{-\infty}\right)$. Consequently

$$
\left(\omega(n)^{2}-h_{n}^{-2}\right)\left\|\Pi_{n} v\right\|_{H^{-4}}=O\left(n^{-\infty}\right) .
$$

To get (1.2.46) and conclude the proof, we just need to see that $\left\|\Pi_{n} v\right\|_{H^{-4}} \sim$ $n^{-4}\left\|\Pi_{n} v\right\|_{L^{2}} \geq c n^{-4}$. We have, since $v$ is in the unit ball of $F_{n},\left\|\Pi_{n}^{\perp} v\right\|_{H^{1}} \leq$ $C\|v\|_{H^{1}} \leq C n$. Interpolating with (1.2.52), we get $\left\|\Pi_{n}^{\perp} v\right\|_{L^{2}}=O\left(n^{-\infty}\right)$, whence the wanted lower bound, $\left\|\Pi_{n} v\right\|_{L^{2}} \geq c$.

Proof of Theorem 1.2.1. For $n$ large enough, we take for $\left(\varphi_{n}^{1}, \varphi_{n}^{2}\right)$ the orthonormal basis of $E_{n}$ given by Lemma 1.2.6. For small values of $n$, we take any orthonormal basis of $E_{n}$. Remark first that if $\left|n-n^{\prime}\right| \geq c\left(n+n^{\prime}\right)$ for some $c>0$, estimate (1.2.14) holds true. Actually, one has a general estimate

$$
\left|\left\langle\Pi_{n} u, T \Pi_{n^{\prime}} v\right\rangle\right| \leq C_{N}\left\langle n-n^{\prime}\right\rangle^{-N}|a|_{\nu+N}\|u\|_{L^{2}}\|v\|_{L^{2}}
$$


for a fixed $\nu \in \mathbb{R}_{+}$(see for instance [10, Proposition 1.2.2 and Lemma 1.2.3). This implies that if $\left|n-n^{\prime}\right| \geq c\left(n+n^{\prime}\right),\left|\left\langle\varphi_{n}^{j}, T \varphi_{n^{\prime}}^{j^{\prime}}\right\rangle\right|$ is bounded from above by $C_{N}\left(n+n^{\prime}\right)^{-N}|a|_{\nu+N}$, which is better than the wanted estimate (1.2.14). We may thus assume $\left|n-n^{\prime}\right| \leq c\left(n+n^{\prime}\right)$ and $n, n^{\prime}$ large enough. Then using (1.2.47) we get that up to $O\left(\left(n+n^{\prime}\right)^{-\infty}\right)$ terms, $\left\langle\varphi_{n}^{j}, T \varphi_{n^{\prime}}^{j^{\prime}}\right\rangle$ may be written as linear combinations of $I_{-}\left(n, n^{\prime}\right)$ and $I_{+}\left(n, n^{\prime}\right)$. Formulas (1.2.17), (1.2.18) of Proposition 1.2.3 give then (1.2.14). This concludes the proof of the theorem.

\section{Paradifferential Symbolic CalCulus}

The aim of this section is to develop a symbolic calculus, analogous to Bony's paradifferential calculus [4], for symbols defined on a discrete set instead of an open subset of Euclidean space. As will be clear in section 4, we shall need such an extension, as the symbols which will naturally appear in reductions of the quasilinear equation (1.1.4) will be defined on $\mathbb{N}^{p}$, and will not have any nice extension to $\mathbb{R}^{p}$.

2.1. Symbols and quantization. We first fix some notation. We shall consider $G$ a finite dimensional real vector space, and assume given an orthonormal decomposition

$$
L^{2}\left(\mathbb{S}^{1}, G\right)=\bigoplus_{k \geq \tau} E_{k}
$$

where $E_{k}$ is a finite dimensional subspace of dimension $K(k)$ and $\tau \in \mathbb{N}^{*}$. We assume $K(k)$ independent of $k$ for $k$ large enough, and denote by $K$ this value. We assume that each $E_{k}$ is endowed with a nice orthonormal basis $\left(\varphi_{k}^{j}\right)_{1 \leq j \leq K(k)}$, i.e. an orthonormal basis such that, for any $k, k^{\prime}$, for given pseudo-differential operators $T_{1}, T_{2}$ of order 0 , and for any function $a \in C^{\infty}\left(\mathbb{S}^{1}, \mathbb{R}\right)$, we have estimates of type (1.2.15):

$$
\begin{aligned}
& \left|\partial_{k}^{\alpha}\left(\partial_{k^{\prime}}^{*}\right)^{\beta}\left(\partial_{k}-\partial_{k^{\prime}}^{*}\right)^{\gamma}\left\langle T_{1} \varphi_{k}^{j}, a(x) T_{2} \varphi_{k^{\prime}}^{j^{\prime}}\right\rangle\right| \\
& \quad \leq C\left\langle k-k^{\prime}\right\rangle^{-N}\left(k+k^{\prime}\right)^{-\gamma} \sum_{\ell=0}^{\alpha+\beta+\gamma+N+\nu}\left\|\partial^{\ell} a\right\|_{L^{\infty}},
\end{aligned}
$$

where $1 \leq j \leq K(k), 1 \leq j^{\prime} \leq K\left(k^{\prime}\right)$ and $\nu$ is a fixed positive constant. We shall denote by $\mathcal{E}$ the algebraic direct sum of the $E_{k}$ 's, and will use $\mathcal{E}$ as a space of test functions.

If $n=\left(n_{0}, \ldots, n_{p+1}\right) \in \mathbb{N}_{\tau}^{p+2}$ we define

$$
n^{\prime}=\left(n_{1}, \ldots, n_{p}\right),\left|n^{\prime}\right|=\max \left(n_{1}, \ldots, n_{p}\right) .
$$

Moreover, if $n_{i}$ is such that $n_{i}=\max \left(n_{0}, \ldots, n_{p+1}\right)$ we set

$$
\max _{2}\left(n_{0}, \ldots, n_{p+1}\right)=\max \left(\left\{n_{0}, \ldots, n_{p+1}\right\}-\left\{n_{i}\right\}\right)
$$

and if $n_{j}, j \neq i$, is such that $n_{j}=\max _{2}\left(n_{0}, \ldots, n_{p+1}\right)$ we define

$$
\begin{aligned}
& \mu\left(n_{0}, \ldots, n_{p+1}\right)=\max \left(\left\{n_{0}, \ldots, n_{p+1}\right\}-\left\{n_{i}, n_{j}\right\}\right), \\
& S\left(n_{0}, \ldots, n_{p+1}\right)=\left|n_{i}-n_{j}\right|+\mu\left(n_{0}, \ldots, n_{p+1}\right) .
\end{aligned}
$$


By convention, we set $\max _{2} n_{0}=1, \mu\left(n_{0}, n_{1}\right)=1$. We denote by $\mathbb{K}$ either $\mathbb{R}$ or $\mathbb{C}$ and by $\Pi_{k}$ the orthogonal projector from $L^{2}\left(\mathbb{S}^{1}, G \otimes \mathbb{K}\right)$ to $E_{k} \otimes \mathbb{K}$ and set

$$
\begin{gathered}
\mathcal{F}_{k}: L^{2}\left(\mathbb{S}^{1}, G \otimes \mathbb{K}\right) \longrightarrow \mathbb{K}^{K(k)}, \\
u \rightarrow\left(\left\langle u, \varphi_{k}^{j}\right\rangle\right)_{1 \leq j \leq K(k)} .
\end{gathered}
$$

Then $\mathcal{F}_{k}$ is an isometry when restricted to $E_{k} \otimes \mathbb{K}$, if we endow $\mathbb{K}^{K(k)}$ with the $\ell^{2}$ norm. We denote by $\mathcal{F}_{k}^{*}$ the adjoint of $\mathcal{F}_{k}$ from $\left(\mathbb{K}^{K(k)}\right)^{*} \simeq \mathbb{K}^{K(k)}$ to $\left(L^{2}\right)^{\prime} \simeq L^{2}$. We have for $V=\left(V_{j}\right)_{1 \leq j \leq K(k)} \in \mathbb{K}^{K(k)}$,

$$
\mathcal{F}_{k}^{*} V=\sum_{j=1}^{K(k)} V_{j} \varphi_{k}^{j}(x)
$$

and the relations

$$
\mathcal{F}_{k}^{*}=\Pi_{k} \circ \mathcal{F}_{k}^{*}, \Pi_{k}=\mathcal{F}_{k}^{*} \circ \mathcal{F}_{k}, \mathcal{F}_{k} \circ \mathcal{F}_{k}^{*}=\operatorname{Id}_{\mathbb{K}^{K(k)}}, \mathcal{F}_{k}=\mathcal{F}_{k} \circ \Pi_{k} .
$$

If $U=\left(u_{1}, \ldots, u_{p}\right) \in\left(L^{2}\right)^{p}$ and $n^{\prime}=\left(n_{1}, \ldots, n_{p}\right) \in \mathbb{N}_{\tau}^{p}$ we denote

$$
\Pi_{n^{\prime}} U=\left(\Pi_{n_{1}} u_{1}, \ldots, \Pi_{n_{p}} u_{p}\right) .
$$

We shall always denote by $\|\cdot\|$ the $\mathcal{L}\left(\ell^{2}, \ell^{2}\right)$ norm of linear maps between Euclidean spaces (or the corresponding norm of matrices). Let us define the first class of symbols we shall use.

Definition 2.1.1. Let $d \in \mathbb{R}, \nu \in \mathbb{R}_{+}, p \in \mathbb{N}, N_{0} \in \mathbb{N}^{*}$ be given. We denote by $\Sigma_{p, N_{0}}^{d, \nu}$ the space of maps

$$
\begin{aligned}
& \left(u_{1}, \ldots, u_{p}, n_{0}, n_{p+1}\right) \longrightarrow a\left(u_{1}, \ldots, u_{p} ; n_{0}, n_{p+1}\right), \\
& \mathcal{E} \times \cdots \times \mathcal{E} \times \mathbb{N}_{\tau} \times \mathbb{N}_{\tau} \longrightarrow \mathcal{L}\left(\mathbb{K}^{K\left(n_{p+1}\right)}, \mathbb{K}^{K\left(n_{0}\right)}\right)
\end{aligned}
$$

such that $a$ is $\mathbb{R}$-p-linear in $\left(u_{1}, \ldots, u_{p}\right)$ and satisfies for some $\left.\delta \in\right] 0,1[$ the conditions:

(i) $\delta$ For any $U=\left(u_{1}, \ldots, u_{p}\right) \in \mathcal{E}^{p}$, any $n=\left(n_{0}, n^{\prime}, n_{p+1}\right) \in \mathbb{N}_{\tau}^{p+2}$ (with $n^{\prime}=$ $\left.\left(n_{1}, \ldots, n_{p}\right)\right), a\left(\Pi_{n^{\prime}} U ; n_{0}, n_{p+1}\right) \equiv 0$ unless

$$
\left|n^{\prime}\right| \leq \delta\left(n_{0}+n_{p+1}\right) \text { and }\left|n_{0}-n_{p+1}\right| \leq \delta\left(n_{0}+n_{p+1}\right) .
$$

(ii) For any $N \in \mathbb{N}$, any $\alpha, \beta, \gamma \in \mathbb{N}$, there is $C>0$ such that for any $n=$ $\left(n_{0}, n^{\prime}, n_{p+1}\right) \in \mathbb{N}_{\tau}^{p+2}$ as above, any $U=\left(u_{1}, \ldots, u_{p}\right) \in \mathcal{E}^{p}$, one has the estimate

$$
\begin{aligned}
& \| \partial_{n_{0}}^{\alpha}\left(\partial_{n_{p+1}}^{*}\right)^{\beta}\left(\partial_{n_{0}}-\partial_{n_{p+1}}^{*}\right)^{\gamma} a\left(\Pi_{n^{\prime}} U ; n_{0}, n_{p+1}\right) \| \\
& \leq C\left(n_{0}+n_{p+1}\right)^{d-\gamma} \frac{\left|n^{\prime}\right|^{\nu+N+(\alpha+\beta+\gamma) N_{0}}}{\left(\left|n_{0}-n_{p+1}\right|+\left|n^{\prime}\right|\right)^{N}} \prod_{j=1}^{p}\left\|u_{j}\right\|_{L^{2}} .
\end{aligned}
$$

We shall call the symbols in the preceding class paradifferential symbols. We may of course extend (2.1.10) to a $\mathbb{C}$ - $p$-linear map defined on $(\mathcal{E} \otimes \mathbb{C}) \times \cdots \times(\mathcal{E} \otimes \mathbb{C}) \times \mathbb{N}_{\tau} \times \mathbb{N}_{\tau}$.

Remarks. - When we make $\partial_{n_{p+1}}^{*}$ act several times on $a\left(\Pi_{n^{\prime}} U ; n_{0}, n_{p+1}\right)$, we might, for small values of $n_{p+1}$, have to calculate $a$ at integers smaller than $\tau$. We decide to extend $a\left(\cdot ; n_{0}, n_{p+1}\right)$ as 0 for $n_{0}<\tau$ or $n_{p+1}<\tau$.

- When $\left|n^{\prime}\right|$ is bounded, estimate (2.1.12) is similar to the estimate (2.1.2) defining a nice basis. When $\left|n^{\prime}\right| \rightarrow+\infty$, we have an extra loss of powers of $\left|n^{\prime}\right|$, coming 
from $\left\|\partial^{\ell} a\right\|_{L^{\infty}}$ in (2.1.2), and from degenerate ellipticity estimates of some symbols that we shall have to include in our classes.

- When $p=0$, we set by convention $\left|n^{\prime}\right|=1$ in the above definition, and in all forthcoming formulas.

Let us quantize the above symbols.

Definition 2.1.2. For $a \in \Sigma_{p, N_{0}}^{d, \nu}$ and $U=\left(u_{1}, \ldots, u_{p}\right) \in \mathcal{E}^{p}, u_{p+1} \in \mathcal{E}$, we define

$$
\operatorname{Op}(a(U ; \cdot)) u_{p+1}=\sum_{n_{0} \in \mathbb{N}_{\tau}} \sum_{n_{p+1} \in \mathbb{N}_{\tau}} \mathcal{F}_{n_{0}}^{*}\left[a\left(U ; n_{0}, n_{p+1}\right) \mathcal{F}_{n_{p+1}} u_{p+1}\right] .
$$

Let us explain the origin of the above definition. Assume for instance that each $E_{k}$ is one dimensional, spanned by a function $\varphi_{k}$. If $a, u \in L^{2}$, we may write

$$
\begin{aligned}
a u & =\sum_{n_{p+1}} a(x)\left\langle u, \varphi_{n_{p+1}}\right\rangle \varphi_{n_{p+1}} \\
& =\sum_{n_{0}} \sum_{n_{p+1}}\left\langle a \varphi_{n_{p+1}}, \varphi_{n_{0}}\right\rangle\left\langle u, \varphi_{n_{p+1}}\right\rangle \varphi_{n_{0}} \\
& =\sum_{n_{0}} \sum_{n_{p+1}} \mathcal{F}_{n_{0}}^{*}\left[\left\langle a \varphi_{n_{p+1}}, \varphi_{n_{0}}\right\rangle \mathcal{F}_{n_{p+1}} u\right]
\end{aligned}
$$

using (2.1.6), (2.1.7), and the symbol $\left\langle a \varphi_{n_{p+1}}, \varphi_{n_{0}}\right\rangle$ satisfies by (2.1.2) estimates (2.1.12). Condition $(\mathrm{i})_{\delta}$ of Definition 2.1.1, which is not satisfied in this example, comes from the fact that we want to consider paradifferential operators, instead of pseudo-differential ones.

Let us show that operators of order 0 are bounded on $H^{s}$ for $s$ large enough.

Proposition 2.1.3. Let $\nu \in \mathbb{R}_{+}, N_{0} \in \mathbb{N}^{*}$. There exists $s_{0} \in \mathbb{R}$ and for any $s \in \mathbb{R}$, any $d \in \mathbb{R}$, any $p \in \mathbb{N}$, any $a \in \Sigma_{p, N_{0}}^{d, \nu}$, there is a constant $C>0$ such that for any $U=\left(u_{1}, \ldots, u_{p}\right) \in \mathcal{E}^{p}$, any $u_{p+1} \in \mathcal{E}$,

$$
\left\|\mathrm{Op}(a(U ; \cdot)) u_{p+1}\right\|_{H^{s-d}} \leq C \prod_{j=1}^{p}\left\|u_{j}\right\|_{H^{s_{0}}}\left\|u_{p+1}\right\|_{H^{s}}
$$

In particular, $\left(U, u_{p+1}\right) \rightarrow \mathrm{Op}(a(U ; \cdot)) u_{p+1}$ extends as a bounded $(p+1)$-linear map from $\left(H^{s_{0}}\right)^{p} \times H^{s}$ to $H^{s-d}$.

Proof. Since $\|v\|_{H^{s}}^{2} \sim \sum_{n} n^{2 s}\left\|\Pi_{n} v\right\|_{L^{2}}^{2}$, let us estimate $\left\|\Pi_{n_{0}} \mathrm{Op}(a(U ; \cdot)) u_{p+1}\right\|_{L^{2}}$. We get using (2.1.12) and condition $(\mathrm{i})_{\delta}$,

$$
\begin{aligned}
& n_{0}^{-d}\left\|\sum_{n_{p+1}} a\left(U ; n_{0}, n_{p+1}\right) \mathcal{F}_{n_{p+1}} u_{p+1}\right\|_{\ell^{2}} \\
& \leq C \sum_{n_{1}} \cdots \sum_{n_{p+1}} \frac{\left|n^{\prime}\right|^{\nu+N}}{\left(\left|n_{0}-n_{p+1}\right|+\left|n^{\prime}\right|\right)^{N}} \prod_{j=1}^{p} n_{j}^{-s_{0}} n_{p+1}^{-s} c_{n_{p+1}} \prod_{j=1}^{p}\left\|u_{j}\right\|_{H^{s_{0}}}\left\|u_{p+1}\right\|_{H^{s}}
\end{aligned}
$$

with $\left(c_{n_{p+1}}\right)_{n_{p+1}}$ in the unit ball of $\ell^{2}$. Moreover, by condition $(\mathrm{i})_{\delta}$ of Definition 2.1.1, we have $n_{p+1} \sim n_{0}$ on the summation. Consequently, if we take $N>1$ and $s_{0}$ large enough relative to $\nu$, we obtain an estimate by $C n_{0}^{-s} c_{n_{0}}^{\prime}$ for a new $\ell^{2}$-sequence $\left(c_{n_{0}}^{\prime}\right)_{n_{0}}$, which is the wanted conclusion.

We shall now define a class of remainder operators. 
Definition 2.1.4. Let $d \in \mathbb{R}, \nu \in \mathbb{R}_{+}, p \in \mathbb{N}$. We denote by $\mathcal{R}_{p+1}^{d, \nu}$ the space of $(p+1)$-linear maps $M: \mathcal{E} \times \cdots \times \mathcal{E} \rightarrow L^{2}$ such that for any $\ell, N \in \mathbb{N}$, there is $C>0$ such that for any $\left(n_{0}, \ldots, n_{p+1}\right) \in \mathbb{N}_{\tau}^{p+2}$ and any $u_{1}, \ldots, u_{p+1} \in \mathcal{E}$,

$$
\begin{aligned}
\| \Pi_{n_{0}} M & \left(\Pi_{n_{1}} u_{1}, \ldots, \Pi_{n_{p+1}} u_{p+1}\right) \|_{L^{2}} \\
& \leq C n_{0}^{d} \frac{\max _{2}\left(n_{1}, \ldots, n_{p+1}\right)^{\nu+\ell}}{\max \left(n_{1}, \ldots, n_{p+1}\right)^{\ell}} \frac{\mu\left(n_{0}, \ldots, n_{p+1}\right)^{N}}{S\left(n_{0}, \ldots, n_{p+1}\right)^{N}} \prod_{j=1}^{p+1}\left\|u_{j}\right\|_{L^{2}} .
\end{aligned}
$$

Observe that by definition, $\mathcal{R}_{p+1}^{d, \nu} \subset \mathcal{R}_{p+1}^{0, \nu+d_{+}}$, and that $M$ extends to a $\mathbb{C}-(p+1)$ linear map defined on $(\mathcal{E} \otimes \mathbb{C}) \times \cdots \times(\mathcal{E} \otimes \mathbb{C})$.

Let us show that up to a remainder operator we may always assume in Definition 2.1.1 that condition $(\mathrm{i})_{\delta}$ is satisfied with an arbitrarily small $\delta>0$.

Lemma 2.1.5. Let $d \in \mathbb{R}, \nu \in \mathbb{R}_{+}, p \in \mathbb{N}, N_{0} \in \mathbb{N}^{*}$ be given. There is $\nu^{\prime} \in \mathbb{R}_{+}$such that for any $\left.\delta^{\prime} \in\right] 0,1\left[\right.$, any $a \in \Sigma_{p, N_{0}}^{d, \nu}$, we may find $a_{1} \in \Sigma_{p, N_{0}}^{d, \nu}$, satisfying condition (i) $\delta_{\delta^{\prime}}$ and $R \in \mathcal{R}_{p}^{0, \nu^{\prime}}$, so that for any $U \in \mathcal{E}^{p}, u_{p+1} \in \mathcal{E}$,

$$
\operatorname{Op}(a(U ; \cdot)) u_{p+1}=\operatorname{Op}\left(a_{1}(U ; \cdot)\right) u_{p+1}+R\left(U, u_{p+1}\right) .
$$

Before starting the proof, let us state a lemma that we shall use several times.

Lemma 2.1.6. Assume given a family of real-valued functions $K_{\alpha \beta \gamma}\left(\omega, \omega^{\prime}\right)$ defined on $\mathbb{R}_{+} \times \mathbb{R}_{+}$, such that there are positive constants $C_{\alpha \beta \gamma}$ satisfying

$$
C_{\alpha \beta \gamma}^{-1} K_{\alpha \beta \gamma}\left(\omega, \omega^{\prime}\right) \leq K_{\alpha \beta \gamma}\left(\omega+h, \omega^{\prime}+h^{\prime}\right) \leq C_{\alpha \beta \gamma} K_{\alpha \beta \gamma}\left(\omega, \omega^{\prime}\right)
$$

for any $\omega, \omega^{\prime} \in \mathbb{R}_{+}^{*}$ large enough, any $\left(h, h^{\prime}\right) \in[-1,1]^{2}$. Let $H$ be a smooth function on $\mathbb{R}_{+} \times \mathbb{R}_{+}$satisfying for any $\alpha, \beta, \gamma \in \mathbb{N}$, any $\omega, \omega^{\prime} \in \mathbb{R}_{+}^{*}$,

$$
\left|\partial_{\omega}^{\alpha} \partial_{\omega^{\prime}}^{\beta}\left(\partial_{\omega}+\partial_{\omega^{\prime}}\right)^{\gamma} H\left(\omega, \omega^{\prime}\right)\right| \leq K_{\alpha \beta \gamma}\left(\omega, \omega^{\prime}\right) .
$$

Then, there are constants $C_{\alpha \beta \gamma}^{\prime}$ such that for any $\alpha, \beta, \gamma \in \mathbb{N}$, any $n, n^{\prime} \in \mathbb{N}$ large enough, with $\left|n-n^{\prime}\right| \leq \frac{1}{2}\left(n+n^{\prime}\right)$,

$$
\left|\partial_{n}^{\alpha}\left(\partial_{n^{\prime}}^{*}\right)^{\beta}\left(\partial_{n}-\partial_{n^{\prime}}^{*}\right)^{\gamma} H\left(n, n^{\prime}\right)\right| \leq C_{\alpha \beta \gamma}^{\prime} K_{\alpha \beta \gamma}\left(n, n^{\prime}\right) .
$$

Proof of Lemma 2.1.5, Let $\chi$ be a smooth function, with support close enough to 0 , and equal to one on a neighborhood of zero. Define

$$
a_{1}\left(U ; n_{0}, n_{p+1}\right)=\sum_{n^{\prime}=\left(n_{1}, \ldots, n_{p}\right)} \chi\left(\frac{n_{0}-n_{p+1}}{n_{0}+n_{p+1}}\right) \chi\left(\frac{\left|n^{\prime}\right|}{n_{0}+n_{p+1}}\right) a\left(\Pi_{n^{\prime}} U ; n_{0}, n_{p+1}\right) .
$$

Then condition $(\mathrm{i})_{\delta^{\prime}}$ will be satisfied by $a_{1}$ if Supp $\chi$ is small enough. Moreover, using Lemma 2.1.6, we see that when $\left|n_{0}-n_{p+1}\right| \leq \frac{1}{2}\left(n_{0}+n_{p+1}\right)$,

$$
\begin{aligned}
& \left|\partial_{n_{0}}^{\alpha}\left(\partial_{n_{p+1}}^{*}\right)^{\beta}\left(\partial_{n_{0}}-\partial_{n_{p+1}}^{*}\right)^{\gamma} \chi\left(\frac{n_{0}-n_{p+1}}{n_{0}+n_{p+1}}\right)\right| \leq C_{\alpha \beta \gamma}\left(n_{0}+n_{p+1}\right)^{-\gamma}, \\
& \left|\partial_{n_{0}}^{\alpha}\left(\partial_{n_{p+1}}^{*}\right)^{\beta}\left(\partial_{n_{0}}-\partial_{n_{p+1}}^{*}\right)^{\gamma} \chi\left(\frac{\left|n^{\prime}\right|}{n_{0}+n_{p+1}}\right)\right| \leq C_{\alpha \beta \gamma} \frac{\left|n^{\prime}\right|^{\alpha+\beta+\gamma}}{\left(n_{0}+n_{p+1}\right)^{\alpha+\beta+\gamma}} .
\end{aligned}
$$

Consequently, using also the Leibniz formulas (1.2.6), (1.2.7), we see that the estimates (2.1.12) are satisfied by $a_{1}$. Finally, since $R=\operatorname{Op}\left(a-a_{1}\right)$,

$$
\left\|\Pi_{n_{0}} R\left(\Pi_{n_{1}} u_{1}, \ldots, \Pi_{n_{p+1}} u_{p+1}\right)\right\|_{L^{2}} \leq\left\|\left(a-a_{1}\right)\left(\Pi_{n^{\prime}} U ; n_{0}, n_{p+1}\right)\right\|\left\|u_{p+1}\right\|_{L^{2}}
$$


and since, for the indices to be considered, either $\left|n^{\prime}\right| \geq c\left(n_{0}+n_{p+1}\right)$ or $\left|n_{0}-n_{p+1}\right| \geq$ $c\left(n_{0}+n_{p+1}\right)$, estimate (2.1.12) gives the upper bound

$$
C\left(n_{0}+n_{p+1}\right)^{d-N}\left|n^{\prime}\right|^{\nu+N}
$$

from which (2.1.15) follows, since

$$
\max _{2}\left(n_{1}, \ldots, n_{p+1}\right) \sim\left|n^{\prime}\right|, \mu\left(n_{0}, \ldots, n_{p+1}\right) \sim\left|n^{\prime}\right|, S\left(n_{0}, \ldots, n_{p+1}\right) \leq C\left(n_{0}+n_{p+1}\right)
$$

because of (2.1.11).

Remainder operators act also on Sobolev spaces:

Lemma 2.1.7. Let $s_{0}>1$. There is for any $\nu \in \mathbb{R}_{+}$, any $p \in \mathbb{N}^{*}, s_{1}, s_{2} \in$ $\mathbb{R}, s_{1}+s_{2}>\nu+2$, any $d \in \mathbb{R}$, any $M \in \mathcal{R}_{p+1}^{d, \nu}$, a constant $C>0$ such that for any $u_{1}, \ldots, u_{p+1} \in \mathcal{E}$, any $n_{0} \in \mathbb{N}_{\tau}$, one has the estimate

$$
\left\|\Pi_{n_{0}}\left[M\left(u_{1}, \ldots, u_{p+1}\right)\right]\right\|_{L^{2}}
$$

In particular, $M$ is bounded for any $\theta$ from $H^{s} \times \cdots \times H^{s}$ to $H^{s+\theta-d}$ if $s$ is large enough with respect to $\nu$ and $\theta$ and

$$
\|M(u, \ldots, u)\|_{H^{s+\theta-d}} \leq C\|u\|_{H^{s_{0}}}^{p-1}\|u\|_{H^{s}}^{2} .
$$

Proof. We consider the contribution to $M$ of

$$
M_{1}\left(u_{1}, \ldots, u_{p+1}\right)=\sum_{n_{1} \leq \cdots \leq n_{p+1}} M\left(\Pi_{n_{1}} u_{1}, \ldots, \Pi_{n_{p+1}} u_{p+1}\right) .
$$

Then by Definition 2.1.4,

$$
\left\|\Pi_{n_{0}} M_{1}\left(\Pi_{n_{1}} u_{1}, \ldots, \Pi_{n_{p+1}} u_{p+1}\right)\right\|_{L^{2}} \leq C n_{0}^{d} \frac{n_{p}^{\nu+\ell}}{n_{p+1}^{\ell}} \frac{\mu\left(n_{0}, \ldots, n_{p+1}\right)^{N}}{S\left(n_{0}, \ldots, n_{p+1}\right)^{N}} \prod_{1}^{p+1}\left\|\Pi_{n_{j}} u_{j}\right\|_{L^{2}} .
$$

For the summation for $n_{1} \leq \cdots \leq n_{p+1}$ and $n_{p} \geq n_{0}$, we take $\ell=s_{1}-\nu, N=0$. We get the upper bound

$$
C n_{0}^{d} \sum_{\substack{n_{1} \leq \cdots \leq n_{p+1} \\ n_{p} \geq n_{0}}} n_{p+1}^{-s_{1}-s_{2}+\nu} n_{p-1}^{-s_{0}} \cdots n_{1}^{-s_{0}} \prod_{1}^{p-1}\left\|u_{j}\right\|_{H^{s_{0}}}\left\|u_{p}\right\|_{H^{s_{1}}}\left\|u_{p+1}\right\|_{H^{s_{2}}},
$$

which is bounded by the right hand side of (2.1.19) for $s_{1}+s_{2}>\nu+2, s_{0}>1$. When we sum for $n_{1} \leq \cdots \leq n_{p+1}$ and $n_{p}<n_{0}$, we have $\mu\left(n_{0}, \ldots, n_{p+1}\right)=n_{p}$, $S\left(n_{0}, \ldots, n_{p+1}\right)=\left|n_{0}-n_{p+1}\right|+n_{p}$. We take in (2.1.20) $\ell=s_{1}-\nu$, and get

$$
\begin{array}{r}
C n_{0}^{d} \sum_{\substack{n_{1} \leq \cdots \leq n_{p+1} \\
n_{0}>n_{p}}} n_{1}^{-s_{0}} \cdots n_{p-1}^{-s_{0}} n_{p+1}^{-s_{1}-s_{2}+\nu} n_{p}^{N}\left(\left|n_{0}-n_{p+1}\right|+n_{p}\right)^{-N} \\
\times \prod_{1}^{p-1}\left\|u_{j}\right\|_{H^{s_{0}}}\left\|u_{p}\right\|_{H^{s_{1}}}\left\|u_{p+1}\right\|_{H^{s_{2}}} .
\end{array}
$$


For the sum over $n_{p+1} \geq \frac{1}{2} n_{0}$, we take $N=0$ and get the upper bound (2.1.19). For the sum over $n_{p+1}<\frac{1}{2} n_{0}$, we take $N=s_{1}+s_{2}-\nu$ and get a bound in terms of

$$
\sum_{n_{1} \leq \cdots \leq n_{p+1}<\frac{1}{2} n_{0}} n_{1}^{-s_{0}} \cdots n_{p-1}^{-s_{0}} n_{0}^{-s_{1}-s_{2}+\nu} \leq C n_{0}^{-s_{1}-s_{2}+\nu+2},
$$

whence again (2.1.19).

2.2. Symbolic calculus. We shall prove that the operators we just defined enjoy nice symbolic calculus properties.

Definition 2.2.1. Let $a \in \Sigma_{p, N_{0}}^{d, \nu}$. We denote by $a^{\bullet}$ the symbol defined by

$$
a^{\bullet}\left(U ; n_{0}, n_{p+1}\right)=a\left(U ; n_{p+1}, n_{0}\right)^{*},
$$

where $a^{*}$ means the adjoint of the operator $a\left(U ; n_{p+1}, n_{0}\right)$ acting from $\mathbb{K}^{K\left(n_{0}\right)}$ to $\mathbb{K}^{K\left(n_{p+1}\right)}$.

Remark that since

$$
\left(\partial_{n_{0}}-\partial_{n_{p+1}}^{*}\right)\left[a^{\bullet}\left(U ; n_{0}, n_{p+1}\right)\right]=\left.\left[\left(\partial_{X}-\partial_{Y}^{*}\right) a(U ; X, Y)^{*}\right]\right|_{X=n_{p+1}-1, Y=n_{0}+1}
$$

we get that $a^{\bullet} \in \Sigma_{p, N_{0}}^{d, \nu}$. Moreover, it follows from Definition 2.1.2 that

$$
\operatorname{Op}(a(U ; \cdot))^{*}=\operatorname{Op}\left(a^{\bullet}(U ; \cdot)\right),
$$

where the star denotes here the adjoint of operators from $L^{2}$ to $L^{2}$.

Let us now study composition.

Proposition 2.2.2. (i) Let $\nu \in \mathbb{R}_{+}, N_{0} \in \mathbb{N}^{*}$. There is $\nu^{\prime} \in \mathbb{R}_{+}$and for any $p, q \in \mathbb{N}, d, d^{\prime} \in \mathbb{R}$, for any symbols $a \in \Sigma_{p, N_{0}}^{d, \nu}, b \in \Sigma_{q, N_{0}}^{d^{\prime}, \nu}$ satisfying condition $(i)_{\delta}$ of Definition 2.1.1 with a small enough $\delta>0$, there is a symbol $a \# b \in \Sigma_{p+q, N_{0}}^{d+d^{\prime}, \nu^{\prime}}$ such that for any $U^{\prime}=\left(u_{1}, \ldots, u_{p}\right) \in \mathcal{E}^{p}, U^{\prime \prime}=\left(u_{p+1}, \ldots, u_{p+q}\right) \in \mathcal{E}^{q}$, any $u_{p+q+1} \in \mathcal{E}$,

$$
\operatorname{Op}\left(a\left(U^{\prime} ; \cdot\right)\right) \operatorname{Op}\left(b\left(U^{\prime \prime} ; \cdot\right)\right) u_{p+q+1}=\operatorname{Op}\left(a \# b\left(U^{\prime}, U^{\prime \prime} ; \cdot\right)\right) u_{p+q+1} .
$$

(ii) Assume moreover that for any $U^{\prime}, U^{\prime \prime}$ as above, any large enough $n_{0}, n_{p+1}$, $n_{0}^{\prime}, n_{q+1}^{\prime} \in \mathbb{N}_{\tau}$, the symbols $a\left(U^{\prime} ; n_{0}, n_{p+1}\right)$ and $b\left(U^{\prime \prime} ; n_{0}^{\prime}, n_{q+1}^{\prime}\right)$ commute. Then there is a symbol $c \in \Sigma_{p+q, N_{0}}^{d+d^{\prime}-1, \nu^{\prime}}$ such that

$$
\left[\mathrm{Op}\left(a\left(U^{\prime} ; \cdot\right)\right), \mathrm{Op}\left(b\left(U^{\prime \prime} ; \cdot\right)\right)\right] u_{p+q+1}=\mathrm{Op}\left(c\left(U^{\prime}, U^{\prime \prime} ; \cdot\right)\right) u_{p+q+1}
$$

for any $U^{\prime} \in \mathcal{E}^{p}, U^{\prime \prime} \in \mathcal{E}^{q}, u_{p+q+1} \in \mathcal{E}$.

Proof. (i) Using Definition 2.1.2 and (2.1.8) we get

$$
\begin{aligned}
& \operatorname{Op}\left(a\left(U^{\prime} ; \cdot\right)\right) \operatorname{Op}\left(b\left(U^{\prime \prime} ; \cdot\right)\right) u_{p+q+1} \\
& \quad=\sum_{n_{0}, k, n_{p+q+1} \geq \tau} \mathcal{F}_{n_{0}}^{*}\left[a\left(U^{\prime} ; n_{0}, k\right) b\left(U^{\prime \prime} ; k, n_{p+q+1}\right) \mathcal{F}_{n_{p+q+1}} u_{p+q+1}\right]
\end{aligned}
$$

and we have to check that

$$
(a \# b)\left(U^{\prime}, U^{\prime \prime} ; n_{0}, n_{p+q+1}\right) \stackrel{\text { def }}{=} \sum_{k \geq \tau} a\left(U^{\prime} ; n_{0}, k\right) b\left(U^{\prime \prime} ; k, n_{p+q+1}\right)
$$


belongs to $\Sigma_{p+q, N_{0}}^{d+d^{\prime}, \nu^{\prime}}$ for some $\nu^{\prime}$. If we set $n^{\prime}=\left(n_{1}, \ldots, n_{p}\right), n^{\prime \prime}=\left(n_{p+1}, \ldots, n_{p+q}\right)$ and replace $U^{\prime}$ (resp. $\left.U^{\prime \prime}\right)$ by $\Pi_{n^{\prime}} U^{\prime}$ (resp. $\left.\Pi_{n^{\prime \prime}} U^{\prime \prime}\right)$ we get from condition (i) of Definition 2.1.1 applied to $a, b$,

$$
\begin{aligned}
& \left|n^{\prime}\right| \leq \delta\left(n_{0}+k\right),\left|n^{\prime \prime}\right| \leq \delta\left(k+n_{p+q+1}\right), \\
& \left|n_{0}-k\right| \leq \delta\left(n_{0}+k\right),\left|k-n_{p+q+1}\right| \leq \delta\left(k+n_{p+q+1}\right),
\end{aligned}
$$

which implies that $a \# b$ satisfies (i) $)_{4 \delta}$ if $\delta>0$ is small enough. One has then to check estimate (2.1.12) for $a \# b$. We shall do that in the proof of (ii) below.

(ii) Before starting the proof, let us gather some formulas that we shall use. Let $c(U ; \cdot)$ be a symbol satisfying condition (i) $\delta$ of Definition 2.1.1 with a small enough $\delta>0$. For $h \in \mathbb{Z}$ we have, forgetting the explicit $U$ dependence in the notation, for any $\xi, \eta \in \mathbb{N}$,

$$
c(\xi+h, \eta)-c(\xi, \eta-h)=\mathcal{S}\left(\left(\partial_{\xi}-\partial_{\eta}^{*}\right) c\right)(\xi, \eta ; h),
$$

where $\partial_{\xi}$ (resp. $\partial_{\eta}^{*}$ ) means derivation with respect to the first (resp. second) argument of $c(\xi, \eta)$, and where

$$
\mathcal{S}(c)(\xi, \eta ; h)=\sum_{j=0}^{h-1} c(\xi+h-j-1, \eta-j) .
$$

We shall also denote

$$
\begin{aligned}
(\Delta c)(\xi, \eta ; k) & =c(\xi, \xi+k)-c(\eta-k, \eta) \\
& =\mathcal{S}\left(\left(\partial_{\xi}-\partial_{\eta}^{*}\right) c\right)(\eta-k, \xi+k ; \xi-\eta+k),
\end{aligned}
$$

the last equality following from (2.2.6). By direct computation, one checks that

$$
\begin{aligned}
\partial_{\xi}[\Delta c(\xi, \eta ; k)] & =\left(\left(\partial_{\xi}-\partial_{\eta}^{*}\right) c\right)(\xi, \xi+k+1), \\
\partial_{\eta}^{*}[\Delta c(\xi, \eta ; k)] & =\left(\left(\partial_{\xi}-\partial_{\eta}^{*}\right) c\right)(\eta-k-1, \eta), \\
\left(\partial_{\xi}-\partial_{\eta}^{*}\right)[\Delta c(\xi, \eta ; k)] & =\Delta\left(\left(\partial_{\xi}-\partial_{\eta}^{*}\right) c\right)(\xi, \eta ; k+1)
\end{aligned}
$$

and also that

$$
\begin{aligned}
\partial_{\xi} \mathcal{S}(c)(\xi, \eta ; h) & =\mathcal{S}\left(\partial_{\xi} c\right)(\xi, \eta ; h), \\
\partial_{\eta}^{*} \mathcal{S}(c)(\xi, \eta ; h) & =\mathcal{S}\left(\partial_{\eta}^{*} c\right)(\xi, \eta ; h) .
\end{aligned}
$$

We now consider the symbol of $\left[\mathrm{Op}\left(a\left(U^{\prime} ; \cdot\right)\right), \operatorname{Op}\left(b\left(U^{\prime \prime} ; \cdot\right)\right)\right]$. By (2.2.4) , this is equal to the expression $a \# b\left(U^{\prime}, U^{\prime \prime} ; n_{0}, n_{p+q+1}\right)-b \# a\left(U^{\prime \prime}, U^{\prime} ; n_{0}, n_{p+q+1}\right)$, i.e.

$$
\sum_{k \in \mathbb{N}_{\tau}}\left[a\left(U^{\prime} ; n_{0}, k\right) b\left(U^{\prime \prime} ; k, n_{p+q+1}\right)-b\left(U^{\prime \prime} ; n_{0}, k\right) a\left(U^{\prime} ; k, n_{p+q+1}\right)\right] .
$$

Using the assumption $a b=b a$ and changing indexation, we get for large enough $n_{0}, n_{p+q+1}$,

$$
\begin{aligned}
\sum_{k \in \mathbb{Z}}\left[a ( U ^ { \prime } ; n _ { 0 } , n _ { 0 } + k ) b \left(U^{\prime \prime} ;\right.\right. & \left.n_{0}+k, n_{p+q+1}\right) \\
& \left.-a\left(U^{\prime} ; n_{p+q+1}-k, n_{p+q+1}\right) b\left(U^{\prime \prime} ; n_{0}, n_{p+q+1}-k\right)\right],
\end{aligned}
$$

where because of the assumptions on the support of $a, b$, the $k$ sum is for indices satisfying $|k| \leq c n_{0} \sim c n_{p+q+1}$ for some small constant $c>0$ (see (2.1.11)). We 
may rewrite this using the notation (2.2.6) and (2.2.8):

$$
\begin{aligned}
\sum_{k \in \mathbb{Z}}(\Delta a) & \left(U^{\prime} ; n_{0}, n_{p+q+1} ; k\right) b\left(U^{\prime \prime} ; n_{0}+k, n_{p+q+1}\right) \\
& +\sum_{k \in \mathbb{Z}} a\left(U^{\prime} ; n_{p+q+1}-k, n_{p+q+1}\right) \mathcal{S}\left(\left(\partial_{\xi}-\partial_{\eta}^{*}\right) b\right)\left(U^{\prime \prime} ; n_{0}, n_{p+q+1} ; k\right) .
\end{aligned}
$$

We now prove estimates of type (2.1.12) for each sum over $k$ above. We start with the second sum. If we evaluate the above symbol at $\Pi_{n^{\prime}} U^{\prime}, \Pi_{n^{\prime \prime}} U^{\prime \prime}$ instead of $U^{\prime}, U^{\prime \prime}$, we get from (2.2.7) and (2.1.12),

$$
\begin{aligned}
& \left\|\mathcal{S}\left(\left(\partial_{\xi}-\partial_{\eta}^{*}\right) b\right)\left(\Pi_{n^{\prime \prime}} U^{\prime \prime} ; n_{0}, n_{p+q+1} ; k\right)\right\| \\
& \leq C(1+|k|)\left(n_{0}+n_{p+q+1}\right)^{d^{\prime}-1} \frac{\left|n^{\prime \prime}\right|^{\nu+N+N_{0}}}{\left(\left|n_{0}-n_{p+q+1}+k\right|+\left|n^{\prime \prime}\right|\right)^{N}} \prod_{p+1}^{p+q}\left\|u_{j}\right\|_{L^{2}}
\end{aligned}
$$

Moreover, if we make the derivatives act on $\mathcal{S}\left(\left(\partial_{\xi}-\partial_{\eta}^{*}\right) b\right)$, we have, because of (2.2.10), the same gains and losses as in (2.1.12). On the other hand, by (1.2.8), making a $\partial_{n_{p+q+1}}$ derivative act on $a\left(\Pi_{n^{\prime}} U^{\prime} ; n_{p+q+1}-k, n_{p+q+1}\right)$ provides a gain of one negative power of $n_{p+q+1}$, and a loss of $\left|n^{\prime}\right|^{N_{0}}$. Using (1.2.6), (1.2.7), we thus see that the action of $\partial_{n_{0}}^{\alpha}\left(\partial_{n_{p+q+1}}^{*}\right)^{\beta}\left(\partial_{n_{0}}-\partial_{n_{p+q+1}}^{*}\right)^{\gamma}$ on the general term of the second sum in (2.2.11) is bounded from above by $\prod_{1}^{p+q}\left\|u_{j}\right\|_{L^{2}}$ times

$$
C(1+|k|) n_{p+q+1}^{d_{1}} \frac{\left|n^{\prime}\right|^{\nu+N_{1}+\kappa_{1}}}{\left(|k|+\left|n^{\prime}\right|\right)^{N_{1}}} \frac{\left(n_{0}+n_{p+q+1}\right)^{d_{2}}\left|n^{\prime \prime}\right|^{\nu+N_{2}+\kappa_{2}}}{\left(\left|n_{0}-n_{p+q+1}+k\right|+\left|n^{\prime \prime}\right|\right)^{N_{2}}}
$$

with $d_{1}+d_{2}=d+d^{\prime}-1-\gamma, \kappa_{1}+\kappa_{2}=(\alpha+\beta+\gamma+1) N_{0}, N_{1}, N_{2}$ arbitrary. It is clear that the sum in $k$ satisfying $|k| \ll n_{0} \sim n_{p+q+1}$ of these quantities is bounded from above by

$$
C\left(n_{0}+n_{p+q+1}\right)^{d+d^{\prime}-1-\gamma} \frac{\left(\left|n^{\prime}\right|+\left|n^{\prime \prime}\right|\right)^{2 \nu+3+N_{0}(\alpha+\beta+\gamma+1)+N}}{\left(\left|n_{0}-n_{p+q+1}\right|+\left|n^{\prime}\right|+\left|n^{\prime \prime}\right|\right)^{N}},
$$

which is the (2.1.12)-like estimate wanted (with $\nu$ replaced by $\nu^{\prime}=2 \nu+3+N_{0}$ ). Let us now study the first sum in (2.2.11). It follows from (2.2.7), (2.2.8) and the fact that $|k| \ll n_{0} \sim n_{p+q+1}$ that

$$
\begin{aligned}
& \left\|(\Delta a)\left(\Pi_{n^{\prime}} U^{\prime} ; n_{0}, n_{p+q+1} ; k\right)\right\| \\
& \quad \leq C\left(1+\left|n_{0}-n_{p+q+1}+k\right|\right)\left(n_{0}+n_{p+q+1}\right)^{d-1} \frac{\left|n^{\prime}\right|^{\nu+N+N_{0}}}{\left(|k|+\left|n^{\prime}\right|\right)^{N}} \prod_{1}^{p}\left\|u_{j}\right\|_{L^{2}} .
\end{aligned}
$$

Moreover, if we make $\partial_{n_{0}}-\partial_{n_{p+q+1}}^{*}$ act on $\Delta a$, we gain because of (2.2.9) a decay of type $\left(n_{0}+n_{p+q+1}\right)^{-1}$, and lose $\left|n^{\prime}\right|^{N_{0}}$. In the same way, $\partial_{n_{0}}$ or $\partial_{n_{p+q+1}}^{*}$ lose $\left|n^{\prime}\right|^{N_{0}}$. Similar properties hold true when derivatives act on $b\left(\Pi_{n^{\prime \prime}} U^{\prime \prime} ; n_{0}+k, n_{p+q+1}\right)$. Consequently, using the Leibniz formulas (1.2.6), we see that the action of $\partial_{n_{0}}^{\alpha}\left(\partial_{n_{p+q+1}}^{*}\right)^{\beta}\left(\partial_{n_{0}}-\partial_{n_{p+q+1}}^{*}\right)^{\gamma}$ on the general term of the first sum (2.2.11) gives a quantity bounded from above by an expression similar to (2.2.12), but where $k$ has been replaced by $-k-n_{0}+n_{p+q+1}$. We obtain as above that the $k$-sum is then estimated by (2.2.13). This concludes the proof.

Let us now study composition relative to an inner argument. 
Proposition 2.2.3. Let $d^{\prime} \in \mathbb{R}, \nu \in \mathbb{R}_{+}, N_{0} \in \mathbb{N}^{*}$. There is $\nu^{\prime}=2 \nu+d_{+}^{\prime}+1$ such that for any $p \in \mathbb{N}, q \in \mathbb{N}^{*}, d \in \mathbb{R}$, for any $a \in \Sigma_{q, N_{0}}^{d, \nu}, b \in \Sigma_{p, N_{0}}^{d^{\prime}, \nu}$ satisfying condition (i) of Definition 2.1 .1 with a small enough $\delta>0$, there is $c \in \Sigma_{p+q, N_{0}}^{d, \nu^{\prime}}$ such that for any $U=\left(U^{(1)}, U^{(2)}\right) \in \mathcal{E}^{p+q}$ with $U^{(1)}=\left(u_{1}, \ldots, u_{p}\right), U^{(2)}=\left(u_{p+1}, U^{(3)}\right)$, $U^{(3)}=\left(u_{p+2}, \ldots, u_{p+q}\right)$, for any $u_{p+q+1} \in \mathcal{E}$, one has

$$
\mathrm{Op}\left[a\left(\mathrm{Op}\left(b\left(U^{(1)} ; \cdot\right)\right) u_{p+1}, U^{(3)} ; \cdot\right)\right] u_{p+q+1}=\mathrm{Op}\left(c\left(U^{(1)}, U^{(2)} ; \cdot\right)\right) u_{p+q+1} .
$$

Proof. By Definition 2.1.2 we may write the left hand side as

$$
\begin{aligned}
\sum_{n_{0}} \sum_{n_{p+q}+1} \sum_{k} \sum_{n_{p+1}} \mathcal{F}_{n_{0}}^{*} a\left[\mathcal{F}_{k}^{*} b\left(U^{(1)} ; k, n_{p+1}\right) \mathcal{F}_{n_{p+1}} u_{p+1},\right. \\
\left.U^{(3)} ; n_{0}, n_{p+q+1}\right] \mathcal{F}_{n_{p+q+1}} u_{p+q+1},
\end{aligned}
$$

which is of the form $\operatorname{Op}\left(c\left(U^{(1)}, U^{(2)} ; \cdot\right)\right) u_{p+q+1}$ if we define

$$
\begin{aligned}
& c\left(U^{(1)}, U^{(2)} ; n_{0}, n_{p+q+1}\right) \\
& \quad=\sum_{k} \sum_{n_{p+1}} a\left[\mathcal{F}_{k}^{*} b\left(U^{(1)} ; k, n_{p+1}\right) \mathcal{F}_{n_{p+1}} u_{p+1}, U^{(3)} ; n_{0}, n_{p+q+1}\right] .
\end{aligned}
$$

Let us check that if we denote by $n^{(1)}=\left(n_{1}, \ldots, n_{p}\right), n^{(2)}=\left(n_{p+1}, n^{(3)}\right), n^{(3)}=$ $\left(n_{p+2}, \ldots, n_{p+q}\right), c\left(\Pi_{n^{(1)}} U^{(1)}, \Pi_{n^{(2)}} U^{(2)} ; n_{0}, n_{p+q+1}\right)$ satisfies the conditions of Definition 2.1.1. The support condition $(\mathrm{i})_{2 \delta}$ holds true if $(\mathrm{i})_{\delta}$ is satisfied by $a, b$ with small enough $\delta>0$. Moreover, it is enough to check (2.1.12) when $\alpha=\beta=\gamma=0$. Using the assumption on $a, b$, we get for $\left\|c\left(\Pi_{n^{(1)}} U^{(1)}, \Pi_{n^{(2)}} U^{(2)} ; n_{0}, n_{p+q+1}\right)\right\|$ an upper bound given by the product of $C \prod_{j=1}^{p+q}\left\|u_{j}\right\|_{L^{2}}$ and of

$\sum_{k}\left(n_{0}+n_{p+q+1}\right)^{d} \frac{\left(k+\left|n^{(3)}\right|\right)^{\nu+N_{1}}}{\left(\left|n_{0}-n_{p+q+1}\right|+\left|n^{(3)}\right|+k\right)^{N_{1}}}\left(n_{p+1}+k\right)^{d^{\prime}} \frac{\left|n^{(1)}\right|^{\nu+N_{2}}}{\left(\left|k-n_{p+1}\right|+\left|n^{(1)}\right|\right)^{N_{2}}}$

for any $N_{1}, N_{2}$. Moreover, by condition (i) $\delta$ satisfied by $a, b$, the $k$-summation is made for $n_{p+1} \sim k \ll n_{0} \sim n_{p+q+1}$. We see that taking $N_{2}=0$, we get for the sum the upper bound

$$
C\left(n_{0}+n_{p+q+1}\right)^{d} \frac{\left|\left(n^{(1)}, n^{(2)}\right)\right|^{2 \nu+d_{+}^{\prime}+N_{1}+1}}{\left(\left|n_{0}-n_{p+q+1}\right|+\left|\left(n^{(1)}, n^{(2)}\right)\right|\right)^{N_{1}}},
$$

which gives the wanted conclusion with $\nu^{\prime}=2 \nu+d_{+}^{\prime}+1$.

We shall now study composition of an operator associated to a paradifferential symbol with a remainder operator.

Proposition 2.2.4. Let $p \in \mathbb{N}^{*}, q \in \mathbb{N}, d, d^{\prime} \in \mathbb{R}, \nu \in \mathbb{R}_{+}, N_{0} \in \mathbb{N}^{*}$. There are $\nu^{\prime}=2 \nu+d_{+}^{\prime}+1$ and $\nu^{\prime \prime}=2 \nu+1$ such that for any $a \in \Sigma_{q+1, N_{0}}^{d, \nu}$ satisfying condition (i) $)_{\delta}$ of Definition 2.1.1 with $\delta>0$ small enough, for any $M \in \mathcal{R}_{p}^{d^{\prime}, \nu}$, there are a symbol $b \in \Sigma_{p+q, N_{0}}^{d, \nu^{\prime}}$ and an operator $R \in \mathcal{R}_{p+q+1}^{d+d_{+}^{\prime}, \nu^{\prime \prime}}$, such that for any $U=\left(U^{\prime}, u_{p+q+1}\right) \in \mathcal{E}^{p+q+1}$ with $U^{\prime}=\left(U^{(1)}, U^{(2)}\right), U^{(1)}=\left(u_{1}, \ldots, u_{p}\right), U^{(2)}=$ $\left(u_{p+1}, \ldots, u_{p+q}\right)$,

$$
\mathrm{Op}\left[a\left(M\left(U^{(1)}\right), U^{(2)} ; \cdot\right)\right] u_{p+q+1}=\mathrm{Op}\left(b\left(U^{\prime} ; \cdot\right)\right) u_{p+q+1}+R(U) .
$$


We shall use several times below an inequality established in the proof of Theorem 2.1.4 of [10] (formulas (2.1.10) and (2.1.11) of that paper). We state this result as a separate lemma.

Lemma 2.2.5. Let $\nu_{1}, \nu_{2} \in \mathbb{R}_{+}$. There is, for any $N>1+\max \left(\nu_{1}, \nu_{2}\right)$, a constant $C_{N}>0$ such that for any $n_{0}, \ldots, n_{p+q+1} \in \mathbb{N}$,

$$
\sum_{k} \frac{\mu\left(n_{0}, \ldots, n_{p}, k\right)^{\nu_{1}+N}}{S\left(n_{0}, \ldots, n_{p}, k\right)^{N}} \frac{\mu\left(k, n_{p+1}, \ldots, n_{p+q+1}\right)^{\nu_{2}+N}}{S\left(k, n_{p+1}, \ldots, n_{p+q+1}\right)^{N}}
$$

is bounded from above by

$$
C_{N} \frac{\mu\left(n_{0}, \ldots, n_{p+q+1}\right)^{\nu^{\prime}+N^{\prime}}}{S\left(n_{0}, \ldots, n_{p+q+1}\right)^{N^{\prime}}}
$$

where $N^{\prime}=N-1-\max \left(\nu_{1}, \nu_{2}\right), \nu^{\prime}=\nu_{1}+\nu_{2}+1$.

Proof of Proposition 2.2.4. Let $\chi \in C_{0}^{\infty}(\mathbb{R}), \chi \equiv 1$ close to zero, $0 \leq \chi \leq 1$ with Supp $\chi$ small enough. If for $n=\left(n_{0}, \ldots, n_{p+q+1}\right)$ we set $n^{(1)}=\left(n_{1}, \ldots, n_{p}\right), n^{(2)}=$ $\left(n_{p+1}, \ldots, n_{p+q}\right), n^{\prime}=\left(n^{(1)}, n^{(2)}\right)$, we define

$$
b\left(U^{\prime} ; n_{0}, n_{p+q+1}\right)=\sum_{n^{(1)}} \chi\left(\frac{\left|n^{(1)}\right|}{n_{0}+n_{p+q+1}}\right) a\left(M\left(\Pi_{n^{(1)}} U^{(1)}\right), U^{(2)} ; n_{0}, n_{p+q+1}\right) .
$$

Remark that if Supp $\chi$ is small enough, condition (i) of Definition 2.1.1 will be satisfied by $b$. We use (2.1.12) for $a$ to estimate $\left\|b\left(\Pi_{n^{\prime}} U^{\prime} ; n_{0}, n_{p+q+1}\right)\right\|$ by

$$
C\left(n_{0}+n_{p+q+1}\right)^{d} \sum_{k} \frac{\left(k+\left|n^{(2)}\right|\right)^{\nu+N}}{\left(\left|n_{0}-n_{p+q+1}\right|+k+\left|n^{(2)}\right|\right)^{N}}\left\|\Pi_{k} M\left(\Pi_{n^{(1)}} U^{(1)}\right)\right\|_{L^{2}} \prod_{p+1}^{p+q}\left\|u_{j}\right\|_{L^{2}}
$$

where the summation is made for $k+\left|n^{(2)}\right| \ll n_{0} \sim n_{p+q+1}$, and where moreover $\left|n^{(1)}\right| \ll n_{0} \sim n_{p+q+1}$. In other words, using notation (2.1.5), we may write the first factor in the $k$-sum as

$$
\frac{\mu\left(n_{0}, k, n^{(2)}, n_{p+q+1}\right)^{\nu+N}}{S\left(n_{0}, k, n^{(2)}, n_{p+q+1}\right)^{N}} .
$$

We estimate the second factor using (2.1.15). We get for any $N$ an upper bound given by the product of $\prod_{1}^{p+q}\left\|u_{j}\right\|_{L^{2}}$ and of

$$
C\left(n_{0}+n_{p+q+1}\right)^{d}\left(1+\left|n^{(1)}\right|\right)^{\nu} \sum_{k} \frac{\mu\left(n_{0}, k, n^{(2)}, n_{p+q+1}\right)^{d_{+}^{\prime}+\nu+N}}{S\left(n_{0}, k, n^{(2)}, n_{p+q+1}\right)^{N}} \frac{\mu\left(k, n^{(1)}\right)^{N}}{S\left(k, n^{(1)}\right)^{N}} .
$$

By Lemma 2.2.5, we obtain the bound

$$
C\left(n_{0}+n_{p+q+1}\right)^{d}\left(1+\left|n^{(1)}\right|\right)^{\nu} \frac{\mu\left(n_{0}, n^{(1)}, n^{(2)}, n_{p+q+1}\right)^{d_{+}^{\prime}+\nu+N^{\prime}+1}}{S\left(n_{0}, n^{(1)}, n^{(2)}, n_{p+q+1}\right)^{N^{\prime}}} .
$$

Since we have by assumption $\left|n^{(2)}\right| \ll n_{0} \sim n_{p+q+1}$, and on the support of the cut-off (2.2.18) $\left|n^{(1)}\right| \ll n_{0}$, we see that

$$
\mu\left(n_{0}, n^{(1)}, n^{(2)}, n_{p+q+1}\right)=\left|n^{\prime}\right|, S\left(n_{0}, n^{(1)}, n^{(2)}, n_{p+q+1}\right)=\left|n_{0}-n_{p+q+1}\right|+\left|n^{\prime}\right| .
$$


We thus get for $b$ an estimate of the form (2.1.12) since derivatives are controlled in the same way. The remainder in (2.2.15) will be given by

$$
\begin{aligned}
R(U)=\sum_{n^{0}} \sum_{n^{(1)}} & \sum_{n^{(2)}} \sum_{n_{p+q+1}} \chi_{1}\left(\frac{\left|n^{(1)}\right|}{n_{0}+n_{p+q+1}}\right) \\
& \times \mathcal{F}_{n_{0}}^{*}\left[a\left(\Pi_{k} M\left(\Pi_{n^{(1)}} U^{(1)}\right), \Pi_{n^{(2)}} U^{(2)} ; n_{0}, n_{p+q+1}\right) \mathcal{F}_{n_{p+q+1}} u_{p+q+1}\right],
\end{aligned}
$$

where $\chi_{1}=1-\chi$. The $L^{2}$ norm of $\Pi_{n_{0}} R\left(\Pi_{n^{(1)}} U^{(1)}, \Pi_{n^{(2)}} U^{(2)}, \Pi_{n_{p+q+1}} u_{p+q+1}\right)$ will be bounded from above using Definitions 2.1.1 and 2.1.4 by $\prod_{1}^{p+q+1}\left\|u_{j}\right\|_{L^{2}}$ times

$$
\begin{array}{r}
\sum_{k} \chi_{1}\left(\frac{\left|n^{(1)}\right|}{n_{0}+n_{p+q+1}}\right) \frac{\left(k+\left|n^{(2)}\right|\right)^{\nu+N}}{\left(\left|n_{0}-n_{p+q+1}\right|+\left|n^{(2)}\right|+k\right)^{N}}\left(n_{0}+n_{p+q+1}\right)^{d} \\
\times k^{d^{\prime}} \frac{\left(\max _{2}\left(n^{(1)}\right)\right)^{\nu+\ell}}{\left(\max \left(n^{(1)}\right)\right)^{\ell}} \frac{\mu\left(k, n^{(1)}\right)^{N}}{S\left(k, n^{(1)}\right)^{N}}
\end{array}
$$

and because of condition (i) of Definition 2.1.1, we may restrict the summation to those $k$ satisfying $k+\left|n^{(2)}\right| \ll n_{0} \sim n_{p+q+1}$. Moreover, the cut-off $\chi_{1}$ localizes for $\left|n^{(1)}\right| \geq c n_{0}$. Consequently (2.2.20) will be bounded from above by

$$
C n_{0}^{d+d_{+}^{\prime}} \frac{\left(\max _{2}\left(n^{(1)}\right)\right)^{\nu+\ell}}{\max \left(n_{0}, n^{(1)}, n^{(2)}, n_{p+q+1}\right)^{\ell}} \sum_{k} \frac{\mu\left(n_{0}, k, n^{(2)}, n_{p+q+1}\right)^{\nu+N}}{S\left(n_{0}, k, n^{(2)}, n_{p+q+1}\right)^{N}} \frac{\mu\left(k, n^{(1)}\right)^{N}}{S\left(k, n^{(1)}\right)^{N}} .
$$

Using again Lemma 2.2.5, we get an upper bound

$$
C n_{0}^{d+d_{+}^{\prime}} \frac{\max _{2}\left(n_{1}, \ldots, n_{p+q+1}\right)^{\nu^{\prime \prime}+\ell}}{\max \left(n_{1}, \ldots, n_{p+q+1}\right)^{\ell}} \frac{\mu\left(n_{0}, \ldots, n_{p+q+1}\right)^{N^{\prime \prime}}}{S\left(n_{0}, \ldots, n_{p+q+1}\right)^{N^{\prime \prime}}}
$$

for new values $\nu^{\prime \prime}=2 \nu+1, N^{\prime \prime}$ of $\nu, N$. This is the wanted remainder estimate.

Let us now study the action of an operator on a remainder.

Proposition 2.2.6. Let $p \in \mathbb{N}, q \in \mathbb{N}^{*}, d \in \mathbb{R}, d^{\prime} \in \mathbb{R}, \nu \in \mathbb{R}_{+}, \nu^{\prime} \in \mathbb{R}_{+}, N_{0} \in \mathbb{N}^{*}$. There is $\nu^{\prime \prime}=\nu+\nu^{\prime}+1$ such that for any $a \in \Sigma_{p, N_{0}}^{d, \nu}$, any $M \in \mathcal{R}_{q}^{d^{\prime}, \nu^{\prime}}$, the operator

$$
\left(u_{1}, \ldots, u_{p+q}\right) \rightarrow \operatorname{Op}\left(a\left(u_{1}, \ldots, u_{p} ; \cdot\right)\right) M\left(u_{p+1}, \ldots, u_{p+q}\right)
$$

is in $\mathcal{R}_{p+q}^{d+d^{\prime}, \nu^{\prime \prime}}$.

Proof. We denote by $U^{(1)}=\left(u_{1}, \ldots, u_{p}\right), U^{(2)}=\left(u_{p+1}, \ldots, u_{p+q}\right), n^{(1)}=$ $\left(n_{1}, \ldots, n_{p}\right), n^{(2)}=\left(n_{p+1}, \ldots, n_{p+q}\right)$. The value of operator (2.2.21) at $\left(\Pi_{n^{(1)}} U^{(1)}\right.$, $\left.\Pi_{n^{(2)}} U^{(2)}\right)$ is

$$
\sum_{n_{0}} \sum_{k} \mathcal{F}_{n_{0}}^{*}\left[a\left(\Pi_{n^{(1)}} U^{(1)} ; n_{0}, k\right) \mathcal{F}_{k} M\left(\Pi_{n^{(2)}} U^{(2)}\right)\right] .
$$

We make $\Pi_{n_{0}}$ act on this expression, and compute the $L^{2}$-norm. Using Definitions 2.1.1 and 2.1.4, we get an estimate in terms of the product of $\prod_{1}^{p+q}\left\|u_{j}\right\|_{L^{2}}$ by

$$
C \sum_{k}\left(n_{0}+k\right)^{d} \frac{\left|n^{(1)}\right|^{\nu+N}}{\left(\left|n_{0}-k\right|+\left|n^{(1)}\right|\right)^{N}} k^{d^{\prime}} \frac{\left(\max _{2} n^{(2)}\right)^{\nu^{\prime}+\ell}}{\left(\max n^{(2)}\right)^{\ell}} \frac{\mu\left(k, n^{(2)}\right)^{N}}{S\left(k, n^{(2)}\right)^{N}}
$$

and we have on the support of the summation $k \sim n_{0} \gg\left|n^{(1)}\right|$. 
- If moreover $k \sim n_{0} \gg\left|n^{(2)}\right|$, we get for (2.2.22) an estimate

$$
C \sum_{k} n_{0}^{d+d^{\prime}}\left|n^{(1)}\right|^{\nu} \frac{\left(\max _{2} n^{(2)}\right)^{\nu^{\prime}+N}}{n_{0}^{N}} .
$$

Since we sum for $\left|k-n_{0}\right| \leq c n_{0}$ by condition (i) of Definition 2.1.1 this gives the upper bound

$$
\begin{aligned}
& C n_{0}^{d+d^{\prime}+1+\nu-N}\left(\max _{2} n^{(2)}\right)^{\nu^{\prime}+N} \\
& \quad \leq C n_{0}^{d+d^{\prime}} \frac{\left(\max _{2}\left(n^{(1)}, n^{(2)}\right)\right)^{\nu+\nu^{\prime}+1+\ell^{\prime}}}{\left(\max \left(n^{(1)}, n^{(2)}\right)\right)^{\ell^{\prime}}} \frac{\mu\left(n_{0}, \ldots, n_{p+q}\right)^{N^{\prime}}}{S\left(n_{0}, \ldots, n_{p+q}\right)^{N^{\prime}}}
\end{aligned}
$$

if we take $N=\ell^{\prime}+N^{\prime}+\nu+1$. This is a remainder type estimate.

- If $\left|n^{(2)}\right| \geq c n_{0}$ for some $c>0$, we bound (2.2.22) from above by

$$
C n_{0}^{d+d^{\prime}} \frac{\left(\max _{2}\left(n^{(1)}, n^{(2)}\right)\right)^{\nu^{\prime}+\ell}}{\left(\max \left(n^{(1)}, n^{(2)}\right)\right)^{\ell}} \sum_{k} \frac{\mu\left(n_{0}, n^{(1)}, k\right)^{\nu+N}}{S\left(n_{0}, n^{(1)}, k\right)^{N}} \frac{\mu\left(k, n^{(2)}\right)^{N}}{S\left(k, n^{(2)}\right)^{N}} .
$$

Using again Lemma 2.2.5 to estimate the $k$-sum, we obtain finally in this case

$$
C n_{0}^{d+d^{\prime}} \frac{\left(\max _{2}\left(n^{(1)}, n^{(2)}\right)\right)^{\nu^{\prime}+\ell}}{\left(\max \left(n^{(1)}, n^{(2)}\right)\right)^{\ell}} \frac{\mu\left(n_{0}, n^{(1)}, n^{(2)}\right)^{\nu+N^{\prime}+1}}{S\left(n_{0}, n^{(1)}, n^{(2)}\right)^{N^{\prime}}}
$$

for a new $N^{\prime}$. This implies the wanted remainder estimate.

Proposition 2.2.7. Let $d, d^{\prime} \in \mathbb{R}, \nu, \nu^{\prime} \in \mathbb{R}_{+}$.

(i) Let $p \in \mathbb{N}, q \in \mathbb{N}^{*}, N_{0} \in \mathbb{N}^{*}$. There is $\nu^{\prime \prime}=d_{+}+\nu+\nu^{\prime}+1$ such that for any $a \in \Sigma_{p, N_{0}}^{d, \nu}$, any $M \in \mathcal{R}_{q}^{d^{\prime}, \nu^{\prime}}$, the operator

$$
R\left(u_{1}, \ldots, u_{p+q}\right)=M\left(\mathrm{Op}\left(a\left(u_{1}, \ldots, u_{p} ; \cdot\right)\right) u_{p+1}, u_{p+2}, \ldots, u_{p+q}\right)
$$

belongs to $\mathcal{R}_{p+q}^{d^{\prime}, \nu^{\prime \prime}}$.

(ii) Let $p \in \mathbb{N}^{*}, q \in \mathbb{N}^{*}$. There is $\nu^{\prime \prime}=\nu+\nu^{\prime}+1+d_{+}^{\prime}$ such that for any $M_{1} \in \mathcal{R}_{q}^{d, \nu}, M_{2} \in \mathcal{R}_{p}^{d^{\prime}, \nu^{\prime}}$ the operator

$$
\left(u_{1}, \ldots, u_{p+q-1}\right) \rightarrow M_{1}\left(M_{2}\left(u_{1}, \ldots, u_{p}\right), u_{p+1}, \ldots, u_{p+q-1}\right)
$$

belongs to $\mathcal{R}_{p+q-1}^{d, \nu^{\prime \prime}}$.

Proof. (i) Denoting again $U^{(1)}=\left(u_{1}, \ldots, u_{p}\right), U^{(2)}=\left(u_{p+1}, U^{(3)}\right), U^{(3)}=\left(u_{p+2}\right.$, $\left.\ldots, u_{p+q}\right)$, and using the similar notation $n^{(1)}, n^{(2)}, n^{(3)}$ for the indices, we have to estimate the quantity

$$
\sum_{k} \Pi_{n_{0}} M\left(\mathcal{F}_{k}^{*} a\left(\Pi_{n^{(1)}} U^{(1)} ; k, n_{p+1}\right) \mathcal{F}_{n_{p+1}} u_{p+1}, \Pi_{n^{(3)}} U^{(3)}\right) .
$$

The $L^{2}$-norm of the general term of (2.2.25) is bounded from above by $\prod_{1}^{p+q}\left\|u_{j}\right\|_{L^{2}}$ times

$$
C n_{0}^{d^{\prime}} \frac{\max _{2}\left(k, n^{(3)}\right)^{\nu^{\prime}+\ell}}{\max \left(k, n^{(3)}\right)^{\ell}} \frac{\mu\left(n_{0}, k, n^{(3)}\right)^{N}}{S\left(n_{0}, k, n^{(3)}\right)^{N}}\left(k+n_{p+1}\right)^{d} \frac{\left|n^{(1)}\right|^{\nu+N}}{\left(\left|k-n_{p+1}\right|+\left|n^{(1)}\right|\right)^{N}} .
$$

Moreover the summation is restricted to $\left|n^{(1)}\right| \ll k \sim n_{p+1}$, which allows one to bound this quantity by

$$
C n_{0}^{d^{\prime}} n_{p+1}^{d} \frac{\max _{2}\left(n^{(1)}, n^{(2)}\right)^{\nu^{\prime}+\ell}}{\max \left(n^{(1)}, n^{(2)}\right)^{\ell}} \frac{\mu\left(n_{0}, k, n^{(3)}\right)^{N}}{S\left(n_{0}, k, n^{(3)}\right)^{N}} \frac{\mu\left(k, n^{(1)}, n_{p+1}\right)^{\nu+N}}{S\left(k, n^{(1)}, n_{p+1}\right)^{N}} .
$$


Using again Lemma 2.2.5 to estimate the $k$-sum, we get an expression of the type

$$
C n_{0}^{d^{\prime}} \frac{\max _{2}\left(n^{(1)}, n^{(2)}\right)^{\nu^{\prime \prime}+\ell}}{\max \left(n^{(1)}, n^{(2)}\right)^{\ell}} \frac{\mu\left(n_{0}, n^{(1)}, n^{(2)}\right)^{N}}{S\left(n_{0}, n^{(1)}, n^{(2)}\right)^{N}}
$$

for $\nu^{\prime \prime}=d_{+}+\nu+\nu^{\prime}+1$, and new values of $N, \ell$.

(ii) We need to estimate the $L^{2}$-norm of

$$
\sum_{k} \Pi_{n_{0}} M_{1}\left[\Pi_{k} M_{2}\left(\Pi_{n^{(1)}} U^{(1)}\right), \Pi_{n^{(2)}} U^{(2)}\right]
$$

if we denote here $U^{(1)}=\left(u_{1}, \ldots, u_{p}\right), U^{(2)}=\left(u_{p+1}, \ldots, u_{p+q-1}\right)$ and use similar notation for $n^{(1)}, n^{(2)}$. The $L^{2}$-norm of the general term of (2.2.26) is bounded from above by

$$
C n_{0}^{d} \frac{\max _{2}\left(k, n^{(2)}\right)^{\nu+\ell_{2}}}{\max \left(k, n^{(2)}\right)^{\ell_{2}}} \frac{\mu\left(n_{0}, k, n^{(2)}\right)^{N_{2}}}{S\left(n_{0}, k, n^{(2)}\right)^{N_{2}}} k^{d^{\prime}} \frac{\max _{2}\left(n^{(1)}\right)^{\nu^{\prime}+\ell_{1}}}{\max \left(n^{(1)}\right)^{\ell_{1}}} \frac{\mu\left(k, n^{(1)}\right)^{N_{1}}}{S\left(k, n^{(1)}\right)^{N_{1}}} .
$$

Assume for instance $n_{1} \leq \cdots \leq n_{p}, n_{p+q-1} \leq \cdots \leq n_{p+1}$. The above expression may be written

$$
\begin{aligned}
C n_{0}^{d} k^{d^{\prime}} \frac{n_{p-1}^{\nu^{\prime}+\ell_{1}}}{n_{p}^{\ell_{1}}} & \frac{\max _{2}\left(k, n_{p+2}, n_{p+1}\right)^{\nu+\ell_{2}}}{\max \left(k, n_{p+1}\right)^{\ell_{2}}} \\
& \times \frac{\mu\left(k, n_{p-2}, n_{p-1}, n_{p}\right)^{N_{1}}}{S\left(k, n_{p-2}, n_{p-1}, n_{p}\right)^{N_{1}}} \frac{\mu\left(n_{0}, k, n_{p+3}, n_{p+2}, n_{p+1}\right)^{N_{2}}}{S\left(n_{0}, k, n_{p+3}, n_{p+2}, n_{p+1}\right)^{N_{2}}} .
\end{aligned}
$$

Remark first that, changing eventually the definition of $\ell_{2}$, we can control the $k^{d^{\prime}}$ term by $\max _{2}\left(k, n_{p+2}, n_{p+1}\right)^{d_{+}^{\prime}}$. In the following we thus remove the $k^{d^{\prime}}$ term and replace $\nu$ by $\nu+d_{+}^{\prime}$.

- If $n_{p} \geq \frac{1}{A} n_{p+1}$ for a large enough constant $A>0$, we take $\ell_{1}=\ell, \ell_{2}=0$ and we get an upper bound of the type

$$
C n_{0}^{d} \frac{\max _{2}\left(n^{(1)}, n^{(2)}\right)^{\nu+\nu^{\prime}+d_{+}^{\prime}+\ell}}{\max \left(n^{(1)}, n^{(2)}\right)^{\ell}} \frac{\mu\left(k, n^{(1)}\right)^{N_{1}}}{S\left(k, n^{(1)}\right)^{N_{1}}} \frac{\mu\left(n_{0}, k, n^{(2)}\right)^{N_{2}}}{S\left(n_{0}, k, n^{(2)}\right)^{N_{2}}} .
$$

- If $k \leq A n_{p}<n_{p+1}$, we see that in (2.2.27),

$$
\max _{2}\left(k, n_{p+2}, n_{p+1}\right) \leq A\left(n_{p}+n_{p+2}\right) \leq C \max _{2}\left(n^{(1)}, n^{(2)}\right) .
$$

We take $\ell_{1}=0, \ell_{2}=\ell$ and get again an estimate by (2.2.28).

- If $n_{p}<\frac{1}{A} n_{p+1}$ and $n_{p}<\frac{1}{A} k$, the last but one factor in (2.2.27) may be written $\frac{n_{p-1}^{N_{1}}}{k^{N_{1}}}$. Moreover $\max _{2}\left(k, n_{p+2}, n_{p+1}\right) \leq k$ if we assume $k \geq n_{p+2}$. Taking in (2.2.27) $\ell_{1}=\ell_{2}=0, N_{1}^{\prime}<N_{1}-\nu-d_{+}^{\prime}$, when $n_{p+1} \leq k$, and $\ell_{1}=0, \ell_{2}=N_{1}^{\prime}<N_{1}-\nu-d_{+}^{\prime}$ when $n_{p+1}>k$ we get the upper bound

$$
C n_{0}^{d} \frac{n_{p-1}^{\nu^{\prime}+N_{1}^{\prime}+\nu+d_{+}^{\prime}}}{n_{p+1}^{N_{1}^{\prime}}} \frac{\mu\left(k, n^{(1)}\right)^{N_{1}-N_{1}^{\prime}-\nu-d_{+}^{\prime}}}{S\left(k, n^{(1)}\right)^{N_{1}-N_{1}^{\prime}-\nu-d_{+}^{\prime}}} \frac{\mu\left(n_{0}, k, n^{(2)}\right)^{N_{2}}}{S\left(n_{0}, k, n^{(2)}\right)^{N_{2}}},
$$

which again gives an estimate of type (2.2.28) (changing the definition of the exponents).

If $k<n_{p+2}$, we take in (2.2.27) $\ell_{1}=0$ and get an estimate by

$$
C n_{0}^{d} \frac{n_{p+2}^{\nu+d_{+}^{\prime}+\ell_{2}}}{n_{p+1}^{\ell_{2}}} n_{p-1}^{\nu^{\prime}} \frac{\mu\left(k, n^{(1)}\right)^{N_{1}}}{S\left(k, n^{(1)}\right)^{N_{1}}} \frac{\mu\left(n_{0}, k, n^{(2)}\right)^{N_{2}}}{S\left(n_{0}, k, n^{(2)}\right)^{N_{2}}} .
$$


We get again an estimate of type (2.2.28). To finish the proof, we just have to sum (2.2.28) using again Lemma 2.2.5 to get the wanted upper bound

$$
C n_{0}^{d} \frac{\max _{2}\left(n^{(1)}, n^{(2)}\right)^{\nu^{\prime \prime}+\ell}}{\max \left(n^{(1)}, n^{(2)}\right)^{\ell}} \frac{\mu\left(n_{0}, n^{(1)}, n^{(2)}\right)^{N}}{S\left(n_{0}, n^{(1)}, n^{(2)}\right)^{N}}
$$

with $\nu^{\prime \prime}=\nu+\nu^{\prime}+d_{+}^{\prime}+1$.

\section{SPECIAL PSEUdO-DIFFERENTIAL OPERATORS}

3.1. An introductory example. In addition to the paradifferential symbols introduced in section 2 , we shall need classes of pseudo-differential operators. These classes will be more peculiar than the corresponding paradifferential ones. Let us explain this, and justify their definition through an example. Assume that we are given an orthogonal decomposition $L^{2}=\bigoplus E_{n}$, and assume that $E_{n}$ is one dimensional, spanned by a normalized eigenfunction $\varphi_{n}$. Let $(X, n) \rightarrow b(X, n)$ be a linear real-valued function of $X \in \mathbb{R}$, which is a symbol of order 0 relative to $n\left(\partial_{n}^{\alpha} b(X, n)=O\left(n^{-\alpha}\right), n \rightarrow+\infty\right)$. If $u_{1} \in \mathcal{E}$, we can define the action of the pseudo-differential operator with symbol $b\left(u_{1}, n\right)$ on a function $u_{2}$ by the formula

$$
\sum_{n_{2}} b\left(u_{1}, n_{2}\right)\left\langle u_{2}, \varphi_{n_{2}}\right\rangle \varphi_{n_{2}} .
$$

We denote, for future generalization, by $B(X, n)$ the map from $E_{n}$ to $E_{n}$ given for any fixed $X \in \mathbb{R}$ by

$$
B(X, n): \varphi_{n} \rightarrow b(X, n) \varphi_{n}
$$

so that (3.1.1) may be written, if we remember that the orthogonal projection on $E_{n}$ is given by $\Pi_{n} u=\left\langle u, \varphi_{n}\right\rangle \varphi_{n}$,

$$
\sum_{n_{2}} B\left(u_{1}, n_{2}\right) \Pi_{n_{2}} u_{2} .
$$

Remark also that (3.1.1) may be rewritten

$$
\sum_{n_{0}} \sum_{n_{2}} \varphi_{n_{0}}(x)\left\langle b\left(u_{1}, n_{2}\right) \varphi_{n_{2}}, \varphi_{n_{0}}\right\rangle\left\langle u_{2}, \varphi_{n_{2}}\right\rangle,
$$

i.e. with notation (2.1.6), (2.1.7),

$$
\sum_{n_{0}} \sum_{n_{2}} \mathcal{F}_{n_{0}}^{*} c\left(u_{1} ; n_{0}, n_{2}\right) \mathcal{F}_{n_{2}} u_{2}
$$

with

$$
c\left(u_{1} ; n_{0}, n_{2}\right)=\left\langle b\left(u_{1}, n_{2}\right) \varphi_{n_{2}}, \varphi_{n_{0}}\right\rangle .
$$

In other words, the operator (3.1.1) may be written under the form (2.1.13) with a symbol $c$ which may be proved to satisfy estimates (2.1.12).

Our aim in this third section is to introduce a general class of operators of the form (3.1.1). We shall see that they may be expressed in terms of quantities such as (3.1.5), i.e. from (a sum of) paradifferential operators associated to symbols of the classes $\Sigma_{p, N_{0}}^{d, \nu}$ studied in section 2, up to remainder operators. The interest in operators defined through formula (3.1.1) instead of (3.1.5) is that they obey more explicit calculus rules, in particular for the symbol of the composition of two operators. On the other hand, we do not escape the necessity of introducing more general operators, of the form (3.1.5), since to prove our main theorem, we shall 
have to define from operators of type (3.1.1) more general ones, given by symbols of type (3.1.6).

3.2. Definition and calculus of special symbols. Recall that we denoted at the beginning of subsection 2.1 by $G$ a finite dimensional real vector space. Let $\left(g_{i}\right)_{i}$ be a basis of $G$. We fix a nice basis $\left(\varphi_{n}^{j}\right)_{n, j}$ of $L^{2}\left(\mathbb{S}^{1}, \mathbb{R}\right)$, where $\left(\varphi_{n}^{j}\right)_{j}$ is a basis of the subspace $E_{n}^{\prime}$ generated by the eigenfunctions associated to the eigenvalues $\omega_{-}(n) \leq \omega_{+}(n)$ of $\sqrt{-\Delta+V}$. For $\ell=(j, i)$ we set $\varphi_{n}^{\ell}=\varphi_{n}^{j} \otimes g_{i}$. Then $\left(\varphi_{n}^{\ell}\right)_{\ell}$ is a basis of $E_{n}=E_{n}^{\prime} \otimes G$ and $\left(\varphi_{n}^{\ell}\right)_{n, \ell}$ is a nice basis of $L^{2}\left(\mathbb{S}^{1}, G\right) \simeq L^{2}\left(\mathbb{S}^{1}, \mathbb{R}\right) \otimes G$, and we have $L^{2}\left(\mathbb{S}^{1}, G\right)=\bigoplus_{n \geq \tau} E_{n}$. Of course $\left(\varphi_{n}^{\ell}\right)_{\ell}$ provides also a basis of $E_{n} \otimes \mathbb{C}$ and $\left(\varphi_{n}^{\ell}\right)_{\ell, n}$ is a nice basis of $L^{2}\left(\mathbb{S}^{1}, G \otimes \mathbb{C}\right)$ considered as a $\mathbb{C}$-vector space.

Definition 3.2.1. Let $d \in \mathbb{R}, p \in \mathbb{N}$. We denote by $S_{p}^{d}$ the space of maps

$$
\begin{aligned}
& \left(u_{1}, \ldots, u_{p}, n_{p+1}\right) \rightarrow b\left(u_{1}, \ldots, u_{p}, n_{p+1}\right), \\
& \mathcal{E} \times \cdots \times \mathcal{E} \times \mathbb{N}_{\tau} \longrightarrow \mathcal{L}\left(\mathcal{E}, L^{2}\left(\mathbb{S}^{1}, G \otimes \mathbb{K}\right)\right)
\end{aligned}
$$

such that one can find

- a map

$$
B: G \times \cdots \times G \times \mathbb{N}_{\tau} \rightarrow \mathcal{L}(\mathcal{E} \otimes \mathbb{K}, \mathcal{E} \otimes \mathbb{K}),\left(X_{1}, \ldots, X_{p}, n\right) \rightarrow B\left(X_{1}, \ldots, X_{p}, n\right)
$$

which is for any fixed value of $n, p$-linear in $\left(X_{1}, \ldots, X_{p}\right)$, such that for any $X_{1}, \ldots, X_{p} \in G$, any $n \in \mathbb{N}_{\tau}, B\left(X_{1}, \ldots, X_{p}, n\right)$ is an element of $\mathcal{L}\left(E_{n} \otimes \mathbb{K}, E_{n} \otimes \mathbb{K}\right)$ (extended by zero on $\left.\left(E_{n} \otimes \mathbb{K}\right)^{\perp}\right)$, whose matrix elements in the nice basis $\left(\varphi_{n}^{\ell}\right)_{\ell}$ of $E_{n} \otimes \mathbb{K}$ satisfy for any $\alpha \in \mathbb{N}$,

$$
\left|\partial_{n}^{\alpha} B_{\ell \ell^{\prime}}\left(X_{1}, \ldots, X_{p}, n\right)\right| \leq C_{\alpha} n^{d-\alpha} \prod_{1}^{p}\left|X_{j}\right|_{G} ;
$$

- a family of pseudo-differential operators of order 0 on $\mathbb{S}^{1}, T_{1}, \ldots, T_{p}$, such that one may write for any $u_{1}, \ldots, u_{p} \in \mathcal{E}, n_{p+1} \in \mathbb{N}_{\tau}$

$$
b\left(u_{1}, \ldots, u_{p}, n_{p+1}\right)=B\left(T_{1} u_{1}, \ldots, T_{p} u_{p}, n_{p+1}\right) .
$$

We shall quantize the above operators in the following way:

Definition 3.2.2. Let $b \in S_{p}^{d}$. We define an operator $\widetilde{\mathrm{Op}}(b)$ acting on $\mathcal{E}^{p+1}$ by

$$
\widetilde{\mathrm{Op}}(b)\left(u_{1}, \ldots, u_{p}, \cdot\right) u_{p+1}=\sum_{n_{p+1}} b\left(u_{1}, \ldots, u_{p}, n_{p+1}\right) \Pi_{n_{p+1}} u_{p+1} .
$$

We now want to define from an element of $S_{p}^{d}$ and from a cut-off function a symbol in the class $\Sigma_{p, 1}^{d, \nu}$.

Proposition 3.2.3. Let $\chi \in C_{0}^{\infty}(\mathbb{R})$, $\chi$ even with small enough support, $p \in \mathbb{N}^{*}$. There is $\nu \in \mathbb{R}_{+}$such that for any $d \in \mathbb{R}$, if we define for $b \in S_{p}^{d}, u_{1}, \ldots, u_{p} \in \mathcal{E}$, $n_{0}, n_{p+1} \in \mathbb{N}_{\tau}$,

$$
\begin{aligned}
& b_{\chi}\left(u_{1}, \ldots, u_{p} ; n_{0}, n_{p+1}\right) \\
& \quad=\sum_{n_{1}} \cdots \sum_{n_{p}} \chi\left(\frac{\left|n^{\prime}\right|}{n_{0}+n_{p+1}}\right) \chi\left(\frac{n_{0}-n_{p+1}}{n_{0}+n_{p+1}}\right) \mathcal{F}_{n_{0}} \circ b\left(\Pi_{n^{\prime}} U^{\prime}, n_{p+1}\right) \circ \mathcal{F}_{n_{p+1}}^{*},
\end{aligned}
$$

where $U^{\prime}=\left(u_{1}, \ldots, u_{p}\right), n^{\prime}=\left(n_{1}, \ldots, n_{p}\right)$, then $b_{\chi} \in \Sigma_{p, 1}^{d, \nu}$. When $p=0$, we shall set $b_{\chi}\left(n_{0}, n_{1}\right)=\mathcal{F}_{n_{0}} \circ b\left(n_{1}\right) \circ \mathcal{F}_{n_{1}}^{*}$, which is supported for $n_{0}=n_{1}$. 
Remark. We assume in the statement that $\chi$ is even since this implies when, in (3.2.3), $B(X)$ is a selfadjoint linear map independent of $n_{p+1}$, such that the symbol $b_{\chi}$ defined by (3.2.5) is selfadjoint, i.e. satisfies with the notation (2.2.1) that $b_{\chi}^{\bullet}\left(U^{\prime} ; n_{0}, n_{p+1}\right)=b_{\chi}\left(U^{\prime} ; n_{0}, n_{p+1}\right)$.

Proof of Proposition 3.2.3, Remark first that condition $(\mathrm{i})_{\delta}$ of Definition 2.1.1 is satisfied if $\operatorname{Supp} \chi$ is small enough. Recall that we set $K(n)=\operatorname{dim} E_{n}$. Since $\mathcal{F}_{n}$ sends the basis $\left(\varphi_{n}^{\ell}\right)_{\ell}$ of $E_{n} \otimes \mathbb{K}$ onto the canonical basis of $\mathbb{K}^{K(n)}$, the matrix of $b_{\chi}\left(\Pi_{n^{\prime}} U^{\prime} ; n_{0}, n_{p+1}\right)$ in the canonical basis of $\mathbb{K}^{K\left(n_{p+1}\right)}$ and $\mathbb{K}^{K\left(n_{0}\right)}$ is

$$
\chi\left(\frac{\left|n^{\prime}\right|}{n_{0}+n_{p+1}}\right) \chi\left(\frac{n_{0}-n_{p+1}}{n_{0}+n_{p+1}}\right)\left(\left\langle b\left(\Pi_{n^{\prime}} U^{\prime}, n_{p+1}\right) \varphi_{n_{p+1}}^{\ell_{p+1}}, \varphi_{n_{0}}^{\ell_{0}}\right\rangle\right)_{\ell_{0}, \ell_{p+1}} .
$$

Recall also that for $n_{0}, n_{p+1}$ large enough, the size of this matrix is independent of $n_{0}, n_{p+1}$. Using (2.1.18) to estimate derivatives of the cut-offs, and Leibniz formulas (1.2.6), (1.2.7), we see that we just have to get estimates of type (2.1.12) for the matrix in (3.2.6). Decompose $X_{j} \in G$ on the basis $\left(g_{i}\right)_{i}$ of $G$ as $X_{j}=\sum_{i} X_{j}^{i} g_{i}$. Then the entries of the matrix of the map $B\left(X_{1}, \ldots, X_{p}, n_{p+1}\right)$ in the nice basis $\left(\varphi_{n}^{\ell}\right)_{\ell}$ of $E_{n} \otimes \mathbb{K}$ may be decomposed as

$$
B_{\ell_{p+1}^{\prime} \ell_{p+1}}\left(X_{1}, \ldots, X_{p}, n_{p+1}\right)=\sum_{I} B_{\ell_{p+1}^{\prime} \ell_{p+1}}^{I}\left(n_{p+1}\right) X^{I},
$$

where we denote by $I$ a $p$-tuple $I=\left(i_{1}, \ldots, i_{p}\right)$, by $X^{I}=\prod_{j=1}^{p} X_{j}^{i_{j}}$, and by $B_{\ell_{p+1}^{\prime} \ell_{p+1}}^{I}\left(n_{p+1}\right)$ the quantity $B_{\ell_{p+1}^{\prime} \ell_{p+1}}\left(g_{i_{1}}, \ldots, g_{i_{p}}, n_{p+1}\right)$. By (3.2.3),

$$
\left\langle b\left(\Pi_{n^{\prime}} U^{\prime}, n_{p+1}\right) \varphi_{n_{p+1}}^{\ell_{p+1}}, \varphi_{n_{0}}^{\ell_{0}}\right\rangle=\sum_{\ell_{p+1}^{\prime}} \sum_{I}\left\langle B_{\ell_{p+1}^{\prime} \ell_{p+1}}^{I}\left(n_{p+1}\right)\left(T \Pi_{n^{\prime}} U^{\prime}\right)^{I} \varphi_{n_{p+1}}^{\ell_{p+1}^{\prime}}, \varphi_{n_{0}}^{\ell_{0}}\right\rangle,
$$

where $T \Pi_{n^{\prime}} U^{\prime}=\left(T_{1} \Pi_{n_{1}} u_{1}, \ldots, T_{p} \Pi_{n_{p}} u_{p}\right)$. Since $\ell_{p+1}^{\prime} \in\left\{1, \ldots, K\left(n_{p+1}\right)\right\}$ and $K(n)$ is independent of $n \rightarrow+\infty$, and since $I$ describes also a finite set, we actually just need to estimate each term of the above sum, namely

$$
B_{\ell_{p+1}^{\prime} \ell_{p+1}}^{I}\left(n_{p+1}\right)\left\langle\left(T \Pi_{n^{\prime}} U^{\prime}\right)^{I} \varphi_{n_{p+1}}^{\ell_{p+1}^{\prime}}, \varphi_{n_{0}}^{\ell_{0}}\right\rangle .
$$

We apply inequality (2.1.2) with $T_{1}=T_{2}=$ Id to the bracket. We get the following estimate:

$$
\begin{aligned}
& \mid \partial_{n_{0}}^{\alpha}\left(\partial_{n_{p+1}}^{*}\right)^{\beta}\left(\partial_{n_{0}}-\partial_{n_{p+1}}^{*}\right)^{\gamma}\left\langle\varphi_{n_{0}}^{\ell_{0}},\left(T \Pi_{n^{\prime}} U^{\prime}\right)^{I} \varphi_{n_{p+1}}^{\left.\ell_{p+1}^{\prime}\right\rangle \mid}\right. \\
& \quad \leq C\left\langle n_{0}-n_{p+1}\right\rangle^{-N}\left(n_{0}+n_{p+1}\right)^{-\gamma} \sup _{0 \leq k \leq \alpha+\beta+\gamma+N+\nu}\left\|\partial^{k}\left[\left(T \Pi_{n^{\prime}} U^{\prime}\right)^{I}\right]\right\|_{L^{\infty}}
\end{aligned}
$$

for any $\alpha, \beta, \gamma, N \in \mathbb{N}$. By Sobolev injection, and the $L^{2}$-boundedness of pseudodifferential operators of order 0 , we get for the last term in the above formula the upper bound

$$
C\left(1+\left|n^{\prime}\right|\right)^{\alpha+\beta+\gamma+N+\nu} \prod_{1}^{p}\left\|u_{j}\right\|_{L^{2}}
$$

for a new value of $\nu$. If we combine (3.2.9) with (3.2.8) and (3.2.2), and use the Leibniz formulas (1.2.6), (1.2.7), we see that (3.2.7) satisfies estimate (2.1.12) of the definition of symbols (with $N_{0}=1$ ). This concludes the proof. 
We shall need estimates of type (2.1.12) for some functions of type 3.2.5), but depending on extra parameters. We state these estimates as a corollary of the proof of Proposition 3.2.3.

Corollary 3.2.4. (i) Let $b \in S_{p}^{d}$. One has the following estimate for any indices $n_{0}, n^{\prime}=\left(n_{1}, \ldots, n_{p}\right), n_{p+1}, k$ :

$$
\left\|\Pi_{n_{0}} b\left(\Pi_{n^{\prime}} U^{\prime}, k\right) \Pi_{n_{p+1}}\right\|_{\mathcal{L}\left(L^{2}, L^{2}\right)} \leq C \frac{\left|n^{\prime}\right|^{\nu+N}}{\left(\left|n_{0}-n_{p+1}\right|+\left|n^{\prime}\right|\right)^{N}} k^{d}
$$

for some $\nu \in \mathbb{R}_{+}$, independent of $d$.

(ii) Let $B: G \times \cdots \times G \times \mathbb{N}_{\tau} \times \mathbb{N}_{\tau} \rightarrow \mathcal{L}(\mathcal{E} \otimes \mathbb{K}, \mathcal{E} \otimes \mathbb{K})$ be a function

$$
\left(X_{1}, \ldots, X_{p}, n_{p+1}, k\right) \rightarrow B\left(X_{1}, \ldots, X_{p} ; n_{p+1}, k\right),
$$

p-linear in $\left(X_{1}, \ldots, X_{p}\right)$, and such that $B\left(X_{1}, \ldots, X_{p} ; n_{p+1}, k\right)$ is an element of $\mathcal{L}\left(E_{k} \otimes \mathbb{K}, E_{k} \otimes \mathbb{K}\right)$, whose matrix elements in the nice basis of $E_{k} \otimes \mathbb{K}$ satisfy instead of (3.2.2),

$$
\left|\partial_{n_{p+1}}^{\alpha_{1}} \partial_{k}^{\alpha_{2}} B_{\ell \ell^{\prime}}\left(X_{1}, \ldots, X_{p} ; n_{p+1}, k\right)\right| \leq C\left(n_{p+1}+k\right)^{d-\alpha_{1}-\alpha_{2}} \prod_{1}^{p}\left|X_{j}\right|_{G} .
$$

Define as in (3.2.5),

$$
\begin{aligned}
b_{\chi}\left(u_{1}, \ldots, u_{p}, n_{p+1} ; n_{0}, k\right) \\
\quad=\sum_{n_{1}} \cdots \sum_{n_{p}} \chi\left(\frac{\left|n^{\prime}\right|}{n_{0}+k}\right) \chi\left(\frac{n_{0}-k}{n_{0}+k}\right) \mathcal{F}_{n_{0}} \circ b\left(\Pi_{n^{\prime}} U^{\prime} ; n_{p+1}, k\right) \circ \mathcal{F}_{k}^{*} .
\end{aligned}
$$

Then $b_{\chi}$ satisfies

$$
\begin{array}{r}
\left\|\partial_{n_{0}}^{\alpha}\left(\partial_{k}^{*}\right)^{\beta_{1}}\left(\partial_{n_{p+1}}^{*}\right)^{\beta_{2}}\left(\partial_{n_{0}}-\partial_{k}^{*}-\partial_{n_{p+1}}^{*}\right)^{\gamma} b_{\chi}\left(\Pi_{n^{\prime}} U^{\prime}, n_{p+1} ; n_{0}, k\right)\right\| \\
\leq C\left(n_{0}+k\right)^{d-\gamma-\beta_{2}} \frac{\left|n^{\prime}\right|^{\nu+N+\alpha+\beta_{1}+\beta_{2}+\gamma}}{\left(\left|n_{0}-k\right|+\left|n^{\prime}\right|\right)^{N}} \prod_{1}^{p}\left\|u_{j}\right\|_{L^{2}}
\end{array}
$$

for some $\nu \in \mathbb{R}_{+}$, independent of $d$.

Proof. (i) The left hand side of (3.2.10) equals $\left\|\mathcal{F}_{n_{0}} b\left(\Pi_{n^{\prime}} U^{\prime}, k\right) \mathcal{F}_{n_{p+1}}^{*}\right\|$ by (2.1.8) and (2.1.6), (2.1.7). Consequently (3.2.10) is nothing but (2.1.12) in the case $\alpha=$ $\beta=\gamma=0$, when the symbol $b$ depends on an extra parameter $k$, instead of being a function of $n_{p+1}$ as in (3.2.5). Estimate (3.2.10) follows from (3.2.7) to (3.2.9) in the proof of Proposition 3.2 .3 , in which $B_{\ell_{p+1}^{\prime} \ell_{p+1}}^{I}$ is evaluated at $k$ instead of $n_{p+1}$.

(ii) One has just to replace in the proof of Proposition 3.2.3 the reference to (3.2.2) by the reference to (3.2.11), $k$ playing now the role of $n_{p+1}$. Remark that since in (3.2.12), $\partial_{n_{p+1}}$-derivatives act only on the $B_{\ell_{p+1}^{\prime} \ell_{p+1}}^{I}$ term in (3.2.8), they gain one negative power of $k \sim n_{0}+k$.

Our next task will be to express a quantity of the form $\widetilde{\mathrm{Op}}\left(b\left(u_{1}, \ldots, u_{p}, \cdot\right)\right) u_{p+1}$ in terms of the action of paradifferential operators on $u_{1}, u_{2}, \ldots, u_{p+1}$ and of a remainder operator. This is, in our framework, analogous to Bony's paradecomposition of a product [4].

Proposition 3.2.5. Let $p \in \mathbb{N}^{*}$. Let $\chi \in C_{0}^{\infty}(\mathbb{R}), \chi \equiv 1$ close to zero, with small enough support. There is $\nu \in \mathbb{R}_{+}$and for any $d \in \mathbb{R}$, any symbol $b \in S_{p}^{d}$, a family 
of symbols $b_{j} \in \Sigma_{p, 1}^{0, \nu+d_{+}} j=1, \ldots, p$, and a remainder operator $M \in \mathcal{R}_{p+1}^{0, \nu+d_{+}}$such that for any $u_{1}, \ldots, u_{p+1} \in \mathcal{E}$,

$$
\begin{aligned}
\widetilde{\mathrm{Op}}\left(b\left(u_{1}, \ldots, u_{p}, \cdot\right)\right) u_{p+1}= & \mathrm{Op}\left(b_{\chi}\left(u_{1}, \ldots, u_{p} ; \cdot\right)\right) u_{p+1} \\
& +\sum_{j=1}^{p} \mathrm{Op}\left(b_{j}\left(u_{1}, \ldots, \hat{u}_{j}, \ldots, u_{p+1} ; \cdot\right)\right) u_{j} \\
& +M\left(u_{1}, \ldots, u_{p+1}\right) .
\end{aligned}
$$

When $p=0$, we have $\widetilde{\mathrm{Op}}(b(\cdot)) u_{1}=\mathrm{Op}\left(b_{\chi}(u ; \cdot)\right) u_{1}$.

Proof. We first define the symbols $b_{j}$, and check that they belong to $\Sigma_{p, 1}^{0, \nu+d_{+}}$. Define for $j=1, \ldots, p$,

$$
\chi_{j}\left(n_{0}, \ldots, n_{p+1}\right)=\chi\left(\frac{\left|\left(n_{1}, \ldots, \widehat{n_{j}}, \ldots, n_{p+1}\right)\right|}{n_{0}+n_{j}}\right) \chi\left(\frac{n_{0}-n_{j}}{n_{0}+n_{j}}\right)
$$

so that on Supp $\chi_{j}$ we have

$$
n_{k} \leq c\left(n_{0}+n_{j}\right), k \in\{1, \ldots, p+1\}-\{j\},\left|n_{0}-n_{j}\right| \leq c\left(n_{0}+n_{j}\right)
$$

for a small constant $c>0$. Moreover, $\chi_{j} \equiv 1$ on a domain of type (3.2.15) when $c$ is replaced by some smaller constant. We define a linear map $b_{j}\left(u_{1}, \ldots, \widehat{u_{j}}, \ldots, u_{p+1}\right.$; $\left.n_{0}, n_{j}\right)$ from $\mathbb{K}^{K\left(n_{j}\right)}$ to $\mathbb{K}^{K\left(n_{0}\right)}$ as

$V \rightarrow \sum_{\substack{n_{k} \\ k \in\{1, \ldots, p+1\}-\{j\}}} \chi_{j}\left(n_{0}, \ldots, n_{p+1}\right) \mathcal{F}_{n_{0}}\left[b\left(\Pi_{n_{1}} u_{1}, \ldots, \mathcal{F}_{n_{j}}^{*} V, \ldots, \Pi_{n_{p}} u_{p}, n_{p+1}\right) \Pi_{n_{p+1}} u_{p+1}\right]$.

By (3.2.15), condition (i) $\delta$ of Definition 2.1 .1 will be satisfied if $c>0$ is small enough. We must check the estimates of condition (ii). To simplify notation, take from now on $j=1$, and set $n^{\prime}=\left(n^{\prime \prime}, n_{p+1}\right), n^{\prime \prime}=\left(n_{2}, \ldots, n_{p}\right), U^{\prime}=\left(U^{\prime \prime}, u_{p+1}\right)$, $U^{\prime \prime}=\left(u_{2}, \ldots, u_{p}\right), \Pi_{n^{\prime}} U^{\prime}=\left(\Pi_{n_{2}} u_{2}, \ldots, \Pi_{n_{p+1}} u_{p+1}\right)$. Then for $V \in \mathbb{K}^{K\left(n_{1}\right)}$, $b_{1}\left(\Pi_{n^{\prime}} U^{\prime} ; n_{0}, n_{1}\right) \cdot V$ is the product of the function $\chi_{1}\left(n_{0}, \ldots, n_{p+1}\right)$ by the vector of $\mathbb{K}^{K\left(n_{0}\right)}$ with components

$$
\left\langle b\left(\mathcal{F}_{n_{1}}^{*} V, \Pi_{n^{\prime \prime}} U^{\prime \prime}, n_{p+1}\right) \Pi_{n_{p+1}} u_{p+1}, \varphi_{n_{0}}^{\ell_{0}}\right\rangle_{\ell_{0}} .
$$

We use expression (3.2.3) for $b$ in terms of $B$. Let $\left(V_{\ell_{1}}\right)_{\ell_{1}}$ be the coordinates of $\mathcal{F}_{n_{1}}^{*} V$ on $\left(\varphi_{n_{1}}^{\ell_{1}}\right)_{\ell_{1}}$, i.e. using Einstein's conventions, $\mathcal{F}_{n_{1}}^{*} V=V_{\ell_{1}} \varphi_{n_{1}}^{\ell_{1}}$. We may rewrite (3.2.17) as

$$
\left\langle V_{\ell_{1}} B\left(T_{1} \varphi_{n_{1}}^{\ell_{1}}, T^{\prime \prime} \Pi_{n^{\prime \prime}} U^{\prime \prime}, n_{p+1}\right) \Pi_{n_{p+1}} u_{p+1}, \varphi_{n_{0}}^{\ell_{0}}\right\rangle_{\ell_{0}},
$$

where $T^{\prime \prime} \Pi_{n^{\prime \prime}} U^{\prime \prime}=\left(T_{2} \Pi_{n_{2}} u_{2}, \ldots, T_{p} \Pi_{n_{p}} u_{p}\right)$. In other words, the $\left(\ell_{0}, \ell_{1}\right)$ entry of the matrix of $b_{1}\left(\Pi_{n^{\prime}} U^{\prime} ; n_{0}, n_{1}\right)$ in the canonical basis is

$$
\chi_{1}\left(n_{0}, \ldots, n_{p+1}\right)\left\langle B\left(T_{1} \varphi_{n_{1}}^{\ell_{1}}, T^{\prime \prime} \Pi_{n^{\prime \prime}} U^{\prime \prime}, n_{p+1}\right) \Pi_{n_{p+1}} u_{p+1}, \varphi_{n_{0}}^{\ell_{0}}\right\rangle .
$$

Since $T_{1} \varphi_{n_{1}}^{\ell_{1}}$ is a function with values in the finite dimensional vector space $G$, with basis $\left(g_{i}\right)_{i}$, we decompose it as $\left(T_{1} \varphi_{n_{1}}^{\ell_{1}}\right)^{i} g_{i}$ and write the bracket in (3.2.18) as

$$
\left\langle a_{n^{\prime}, i}(x)\left(T_{1} \varphi_{n_{1}}^{\ell_{1}}\right)^{i}, \varphi_{n_{0}}^{\ell_{0}}\right\rangle
$$

with

$$
a_{n^{\prime}, i}(x)=B\left(g_{i}, T^{\prime \prime} \Pi_{n^{\prime \prime}} U^{\prime \prime}, n_{p+1}\right) \Pi_{n_{p+1}} u_{p+1} .
$$


By (3.2.2), Sobolev injection, and the $L^{2}$ continuity of zero-order pseudo-differential operators, we get for any $k$,

$$
\left\|\partial_{x}^{k} a_{n^{\prime}, i}(x)\right\|_{L^{\infty}} \leq C_{k}\left(1+\left|n^{\prime}\right|\right)^{k+\nu+d_{+}} \prod_{2}^{p+1}\left\|u_{j}\right\|_{L^{2}}
$$

for some fixed $\nu \in \mathbb{R}_{+}$. We apply estimate (2.1.2) to (3.2.19) controlling the right hand side by (3.2.21). If we use estimates of type (2.1.18) for $\chi_{1}$ (replacing in (2.1.18) $n_{p+1}$ by $n_{1}$ ) and the Leibniz formulas (1.2.6), (1.2.7), we see that we get for (3.2.18), estimates of type (2.1.12) as wanted.

We must now prove formula (3.2.13). Compute $\mathrm{Op}\left(b_{j}\left(u_{1}, \ldots, \widehat{u_{j}}, \ldots, u_{p+1} ; \cdot\right)\right) u_{j}$ using definition 2.1.2, we must in the right hand side of (3.2.16) replace $V$ by $\mathcal{F}_{n_{j}} u_{j}$, compose on the left with $\mathcal{F}_{n_{0}}^{*}$, and sum in $n_{0}, n_{j}$. Using (2.1.8), we get

$$
\sum_{n_{0}} \cdots \sum_{n_{p+1}} \chi_{j}\left(n_{0}, \ldots, n_{p+1}\right) \Pi_{n_{0}}\left[b\left(\Pi_{n_{1}} u_{1}, \ldots, \Pi_{n_{p}} u_{p}, n_{p+1}\right) \Pi_{n_{p+1}} u_{p+1}\right] .
$$

Consequently, because of the definition of $b_{\chi}, b_{j}$, the operator $M$ defined by equality (3.2.13) may be written as

$$
\begin{aligned}
& M\left(u_{1}, \ldots, u_{p+1}\right) \\
& \quad=\sum_{n_{0}} \cdots \sum_{n_{p+1}} \tilde{\chi}\left(n_{0}, \ldots, n_{p+1}\right) \Pi_{n_{0}}\left[b\left(\Pi_{n_{1}} u_{1}, \ldots, \Pi_{n_{p}} u_{p}, n_{p+1}\right) \Pi_{n_{p+1}} u_{p+1}\right],
\end{aligned}
$$

where $\tilde{\chi}$ cuts off outside a neighborhood of the region where one of the $\chi_{j} j=$ $1, \ldots, p+1$ equals one. In other words, $\tilde{\chi}$ is supported inside

$$
\begin{aligned}
\bigcap_{j=1}^{p+1}\left\{\left(n_{0}, \ldots, n_{p+1}\right) ;\left|n_{0}-n_{j}\right|\right. & \geq c\left(n_{0}+n_{j}\right) \\
& \text { or } \left.\exists k \in\{1, \ldots, p+1\}-\{j\} \text { with } n_{k} \geq c n_{0}\right\}
\end{aligned}
$$

for some small $c>0$. We estimate the $L^{2}$-norm of $\Pi_{n_{0}} M\left(\Pi_{n_{1}} u_{1}, \ldots, \Pi_{n_{p+1}} u_{p+1}\right)$ i.e. of the general term of (3.2.22). Using (3.2.3), we must bound

$$
\left|\tilde{\chi}\left(n_{0}, \ldots, n_{p+1}\right)\right|\left\|\Pi_{n_{0}} B\left(T_{1} \Pi_{n_{1}} u_{1}, \ldots, T_{p} \Pi_{n_{p}} u_{p}, n_{p+1}\right) \Pi_{n_{p+1}} u_{p+1}\right\|_{L^{2}}
$$

or equivalently the product of $\left|\tilde{\chi}\left(n_{0}, \ldots, n_{p+1}\right)\right|$ by

$$
\left\langle B\left(T_{1} \Pi_{n_{1}} u_{1}, \ldots, T_{p} \Pi_{n_{p}} u_{p}, n_{p+1}\right) \Pi_{n_{p+1}} u_{p+1}, \Pi_{n_{0}} u_{0}\right\rangle
$$

for any $u_{0} \in L^{2}$ of norm 1 . If for instance $n_{1}$ and $n_{2}$ are the largest two among $n_{0}, \ldots, n_{p+1}$, we decompose again for $j=1,2$,

$$
T_{j} \Pi_{n_{j}} u_{j}=\sum_{\ell_{j}} \sum_{i_{j}}\left\langle u_{j}, \varphi_{n_{j}}^{\ell_{j}}\right\rangle\left(T_{j} \varphi_{n_{j}}^{\ell_{j}}\right)^{i_{j}} g_{i_{j}},
$$

where $h^{i_{j}}$ denotes the $i_{j}$ th coordinate of an element of $G$ on the basis $\left(g_{k}\right)_{k}$. We set $n^{\prime \prime}=\left(n_{3}, \ldots, n_{p}\right), n^{\prime}=\left(n^{\prime \prime}, n_{p+1}\right)$ and define

$$
a_{i_{1} i_{2} n^{\prime}}(x)=B\left(g_{i_{1}}, g_{i_{2}}, T^{\prime \prime} \Pi_{n^{\prime \prime}} U^{\prime \prime}, n_{p+1}\right) \Pi_{n_{p+1}} u_{p+1} .
$$

Then (3.2.25) may be written as the sum in $\ell_{1}, \ell_{2}, i_{1}, i_{2}$ of

$$
\left\langle u_{1}, \varphi_{n_{1}}^{\ell_{1}}\right\rangle\left\langle u_{2}, \varphi_{n_{2}}^{\ell_{2}}\right\rangle\left\langle a_{i_{1} i_{2} n^{\prime}}(x)\left(T_{1} \varphi_{n_{1}}^{\ell_{1}}\right)^{i_{1}}\left(T_{2} \varphi_{n_{2}}^{\ell_{2}}\right)^{i_{2}}, \Pi_{n_{0}} u_{0}\right\rangle .
$$


The last bracket is estimated by (2.1.2). Using Sobolev injections to control the $L^{\infty}$ norms of derivatives of $a_{i_{1} i_{2} n^{\prime}}$ and (3.2.2), we may bound the modulus of (3.2.26) by

$$
C\left\langle n_{1}-n_{2}\right\rangle^{-N}\left(1+n_{0}+\left|n^{\prime}\right|\right)^{\nu+N} n_{p+1}^{d} \prod_{\ell=0}^{p+1}\left\|u_{\ell}\right\|_{L^{2}}
$$

for any $N$ and some fixed $\nu$. Since $i_{1}, i_{2}, \ell_{1}, \ell_{2}$ in (3.2.26) run in a finite set of indices, we get the same estimate for (3.2.25). Consequently, when the largest two among $n_{0}, \ldots, n_{p+1}$ are among $\left\{n_{1}, \ldots, n_{p}\right\}$, we have for (3.2.24) an upper bound

$$
C n_{p+1}^{d} \frac{\mu\left(n_{0}, \ldots, n_{p+1}\right)^{\nu+N}}{S\left(n_{0}, \ldots, n_{p+1}\right)^{N}} \prod_{1}^{p+1}\left\|u_{j}\right\|_{L^{2}}
$$

for any $N$. One checks in the same way that this formula holds true when one at least of the largest two among $\left(n_{0}, \ldots, n_{p+1}\right)$ equals $n_{0}$ or $n_{p+1}$. To conclude the proof, we have to show that estimate (3.2.27), together with the support conditions (3.2.23), implies the upper bound

$$
C \frac{\max _{2}\left(n_{1}, \ldots, n_{p+1}\right)^{d_{+}+\ell+\nu}}{\max \left(n_{1}, \ldots, n_{p+1}\right)^{\ell}} \frac{\mu\left(n_{0}, \ldots, n_{p+1}\right)^{N}}{S\left(n_{0}, \ldots, n_{p+1}\right)^{N}}
$$

for any $\ell, N$. If there is $c_{1}>0$ with $\max _{2}\left(n_{1}, \ldots, n_{p+1}\right) \geq c_{1} \max \left(n_{1}, \ldots, n_{p+1}\right)$, this is trivial. Assume now

$$
\max _{2}\left(n_{1}, \ldots, n_{p+1}\right)<c_{1} \max \left(n_{1}, \ldots, n_{p+1}\right) .
$$

If, for instance, $n_{p+1}=\max \left(n_{1}, \ldots, n_{p+1}\right)$, we have $n_{p+1} \geq \frac{1}{c_{1}} n_{j}, j=1, \ldots, p$. Assume moreover $\left|n_{0}-n_{p+1}\right| \geq c\left(n_{0}+n_{p+1}\right)$, where $c>0$ is the constant of (3.2.23). Then, if $c_{1}$ is small enough,

$$
S\left(n_{0}, \ldots, n_{p+1}\right) \geq c^{\prime}\left(n_{0}+n_{p+1}\right)
$$

and inequality (3.2.27) implies (3.2.28). We are thus reduced to the case when $\left|n_{0}-n_{p+1}\right|<c\left(n_{0}+n_{p+1}\right)$. By (3.2.23) we must then have $n_{k} \geq c n_{0} \sim c n_{p+1}$ for some $k \in\{1, \ldots, p\}$. This implies again that $\max _{2}\left(n_{1}, \ldots, n_{p+1}\right) \sim \max \left(n_{1}, \ldots\right.$, $\left.n_{p+1}\right)$ and the conclusion follows.

We shall now study symbolic properties of elements in $S_{p}^{d}$. To be able to get for the symbol of a composition a more explicit formula than the one of the proof of Proposition 2.2.2 (ii), we shall have to limit ourselves to symbols which are "scalar" according to the following definition.

Definition 3.2.6. Let $d \in \mathbb{R}, p \in \mathbb{N}$. We denote by $S_{p, \mathrm{sc}}^{d}$ the space of maps

$$
\begin{aligned}
&\left(u_{1}, \ldots, u_{p}, n_{p+1}\right) \rightarrow b\left(u_{1}, \ldots, u_{p}, n_{p+1}\right), \\
& \mathcal{E} \times \cdots \times \mathcal{E} \times \mathbb{N}_{\tau} \rightarrow \mathcal{L}\left(\mathcal{E} \otimes \mathbb{K}, L^{2}\left(\mathbb{S}^{1}, G \otimes \mathbb{K}\right)\right)
\end{aligned}
$$

such that there is

- a function

$B_{s}: G \times \cdots \times G \times \mathbb{N}_{\tau} \rightarrow \mathcal{L}(G \otimes \mathbb{K}, G \otimes \mathbb{K}),\left(X_{1}, \ldots, X_{p}, n\right) \rightarrow B_{s}\left(X_{1}, \ldots, X_{p}, n\right)$, $p$-linear in $\left(X_{1}, \ldots, X_{p}\right)$, satisfying for any $\alpha \in \mathbb{N}$,

$$
\left|\partial_{n}^{\alpha} B_{s}\left(X_{1}, \ldots, X_{p}, n\right)\right| \leq C_{\alpha} n^{d-\alpha} \prod_{1}^{p}\left|X_{j}\right|_{G} ;
$$


- a map

$B_{\infty}: G \times \cdots \times G \times \mathbb{N}_{\tau} \rightarrow \mathcal{L}(G \otimes \mathbb{K}, G \otimes \mathbb{K}),\left(X_{1}, \ldots, X_{p}, n\right) \rightarrow B_{\infty}\left(X_{1}, \ldots, X_{p}, n\right)$, $p$-linear in $\left(X_{1}, \ldots, X_{p}\right)$, such that for any $X_{1}, \ldots, X_{p} \in G$, and any $n \in \mathbb{N}$, $B_{\infty}\left(X_{1}, \ldots, X_{p}, n\right)$ is an element of $\mathcal{L}\left(E_{n} \otimes \mathbb{K}, E_{n} \otimes \mathbb{K}\right)$ whose matrix elements in the nice basis $\left(\varphi_{n}^{\ell}\right)_{\ell}$ of $E_{n}$ satisfy for any $N \in \mathbb{N}$,

$$
\left|B_{\infty, \ell \ell^{\prime}}\left(X_{1}, \ldots, X_{p}, n\right)\right| \leq C_{N} n^{-N} \prod\left|X_{j}\right|_{G} ;
$$

- a family of pseudo-differential operators of order 0 on $\mathbb{S}^{1}, T_{1}, \ldots, T_{p}$ such that one may write for any $u_{1}, \ldots, u_{p} \in \mathcal{E}, n_{p+1} \in \mathbb{N}_{\tau}$,

$$
\begin{aligned}
b\left(u_{1}, \ldots, u_{p}, n_{p+1}\right)= & B_{s}\left(T_{1} u_{1}, \ldots, T_{p} u_{p}, n_{p+1}\right) \otimes \operatorname{Id}_{E_{n_{p+1}}^{\prime} \otimes \mathbb{K}} \\
& +B_{\infty}\left(T_{1} u_{1}, \ldots, T_{p} u_{p}, n_{p+1}\right) .
\end{aligned}
$$

Note that an element of $S_{p, \mathrm{sc}}^{d}$ is in particular an element of $S_{p}^{d}$ as shown by (3.2.32). In the sequel, we shall have to work with $G=\mathbb{K}^{2}$. In this case, $B_{s}$ can be identified with a $2 \times 2$ matrix and the first term on the right hand side of (3.2.32) may be written

$$
\left[\begin{array}{ll}
B_{s, 11} \otimes \operatorname{Id}_{E_{n_{p+1}}^{\prime} \otimes \mathbb{K}} & B_{s, 12} \otimes \operatorname{Id}_{E_{n_{p+1}}^{\prime} \otimes \mathbb{K}} \\
B_{s, 21} \otimes \operatorname{Id}_{E_{n_{p+1}}^{\prime}} \otimes \mathbb{K} & B_{s, 22} \otimes \operatorname{Id}_{E_{n_{p+1}}^{\prime} \otimes \mathbb{K}}^{\prime}
\end{array}\right],
$$

i.e. elements of $S_{p, \mathrm{sc}}^{d}$ are given, up to a perturbation of order $-\infty$, by a matrix in which each block is a scalar operator acting on $E_{n}^{\prime} \otimes \mathbb{K}$.

We shall use in the proof of the following proposition the fact that we can make the scalar part of (3.2.32) act not just on $E_{n_{p+1}} \otimes \mathbb{K}$ but as well on any $E_{k} \otimes \mathbb{K}$ (replacing $\cdot \otimes \operatorname{Id}_{E_{n_{p+1}}^{\prime} \otimes \mathbb{K}}$ by $\left.\cdot \otimes \operatorname{Id}_{E_{k}^{\prime} \otimes \mathbb{K}}\right)$.

Proposition 3.2.7. (i) Let $p, q \in \mathbb{N}$. Let $\chi \in C_{0}^{\infty}(\mathbb{R}), \chi \equiv 1$ close to zero, and assume that $\operatorname{Supp} \chi$ is small enough. There is $\nu \in \mathbb{R}$ and for any $d, d^{\prime} \in \mathbb{R}$, for any symbols $a \in S_{q, \mathrm{sc}}^{d}, b \in S_{p, \mathrm{sc}}^{d^{\prime}}$ there are a symbol $e \in \Sigma_{p+q, 1}^{d+d^{\prime}-1, \nu}$ and a remainder operator $M \in \mathcal{R}_{p+q+1}^{d+d^{\prime}, \nu}$ such that for any $U=\left(U^{\prime}, U^{\prime \prime}\right)$ with $U^{\prime}=\left(u_{1}, \ldots, u_{q}\right) \in \mathcal{E}^{q}$, $U^{\prime \prime}=\left(u_{q+1}, \ldots, u_{p+q}\right) \in \mathcal{E}^{p}$, any $u_{p+q+1} \in \mathcal{E}$, one has

$$
\begin{aligned}
\mathrm{Op}\left(a_{\chi}\left(U^{\prime} ; \cdot\right)\right) \operatorname{Op}\left(b_{\chi}\left(U^{\prime \prime} ; \cdot\right)\right) u_{p+q+1}= & \mathrm{Op}\left((a \circ b)_{\chi}(U ; \cdot)\right) u_{p+q+1} \\
& +\mathrm{Op}(e(U ; \cdot)) u_{p+q+1} \\
& +M\left(u_{1}, \ldots, u_{p+q+1}\right),
\end{aligned}
$$

where $a_{\chi}, b_{\chi}$ are defined in terms of $a, b$ by (3.2.5), and $a \circ b$ stands for the symbol associated to the composition $A \circ B$ of the linear maps defining $a, b$ through (3.2.3).

(ii) Assume moreover that $\chi$ is even and that $a \in S_{q, \mathrm{sc}}^{d}$ satisfies $a\left(U^{\prime} ; \cdot\right)^{*}=$ $a\left(U^{\prime} ; \cdot\right)$. Then there is a symbol $e \in \Sigma_{q, 1}^{d-1, \nu}$ such that

$$
\operatorname{Op}\left(a_{\chi}\left(U^{\prime} ; \cdot\right)\right)^{*}-\operatorname{Op}\left(a_{\chi}\left(U^{\prime} ; \cdot\right)\right)=\operatorname{Op}\left(e\left(U^{\prime} ; \cdot\right)\right)
$$

for any $U^{\prime} \in \mathcal{E}^{q}$.

Proof. (i) We decompose according to (3.2.32) $a=a_{s}+a_{\infty}, b=b_{s}+b_{\infty}$. Then by Proposition 3.2.3, $a_{\infty, \chi}$ and $b_{\infty, \chi}$ belong to $\Sigma_{p, 1}^{-\infty, \nu}$. Consequently by Proposition 2.2.2, their contribution to the left hand side of (3.2.34) may be incorporated to the term $e$ of the right hand side. In the same way, the terms $\left(a_{\infty} \circ b\right)_{\chi}$ or $\left(a \circ b_{\infty}\right)_{\chi}$ on the right hand side may be incorporated to $e$. We may thus assume 
from now on that $a=a_{s}, b=b_{s}$. Using the notation (3.2.14), the definition (3.2.5) of $a_{\chi}, b_{\chi}$, Definition 2.1.2 of quantization of a paradifferential symbol and (2.1.8), we get

$$
\begin{aligned}
\operatorname{Op}\left(a_{\chi}\left(U^{\prime} ; \cdot\right)\right) \operatorname{Op}\left(b_{\chi}\left(U^{\prime \prime} ; \cdot\right)\right) u_{p+q+1} \\
=\sum_{n_{0}} \cdots \sum_{n_{p+q+1}} \sum_{k} \chi_{q+1}\left(n_{0}, n^{\prime}, k\right) \chi_{p+1}\left(k, n^{\prime \prime}, n_{p+q+1}\right) \\
\quad \times \Pi_{n_{0}}\left[a\left(\Pi_{n^{\prime}} U^{\prime}, k\right) \Pi_{k}\left[b\left(\Pi_{n^{\prime \prime}} U^{\prime \prime}, n_{p+q+1}\right) \Pi_{n_{p+q+1}} u_{p+q+1}\right]\right]
\end{aligned}
$$

setting $n^{\prime}=\left(n_{1}, \ldots, n_{q}\right), n^{\prime \prime}=\left(n_{q+1}, \ldots, n_{p+q}\right)$. We write this expression as $I+I I$ where

$$
\begin{aligned}
I=\sum_{n_{0}} \cdots \sum_{n_{p+q+1}} & \sum_{k} \chi_{q+1}\left(n_{0}, n^{\prime}, k\right) \chi_{p+1}\left(k, n^{\prime \prime}, n_{p+q+1}\right) \\
& \times \Pi_{n_{0}}\left[a\left(\Pi_{n^{\prime}} U^{\prime}, n_{p+q+1}\right) \Pi_{k}\left[b\left(\Pi_{n^{\prime \prime}} U^{\prime \prime}, n_{p+q+1}\right) \Pi_{n_{p+q+1}} u_{p+q+1}\right]\right]
\end{aligned}
$$

and

$$
\begin{aligned}
I I=\sum_{n_{0}} \cdots \sum_{n_{p+q+1}} & \sum_{k} \chi_{q+1}\left(n_{0}, n^{\prime}, k\right) \chi_{p+1}\left(k, n^{\prime \prime}, n_{p+q+1}\right) \\
& \times \mathcal{F}_{n_{0}}^{*} \tilde{a}\left(\Pi_{n^{\prime}} U^{\prime}, n_{p+q+1} ; n_{0}, k\right) \tilde{b}\left(\Pi_{n^{\prime \prime}} U^{\prime \prime} ; k, n_{p+q+1}\right) \mathcal{F}_{n_{p+q+1}} u_{p+q+1}
\end{aligned}
$$

with

$$
\begin{aligned}
\tilde{a}\left(\Pi_{n^{\prime}} U^{\prime}, n_{p+q+1} ; n_{0}, k\right) & =\mathcal{F}_{n_{0}} \circ\left[\frac{a\left(\Pi_{n^{\prime}} U^{\prime}, k\right)-a\left(\Pi_{n^{\prime}} U^{\prime}, n_{p+q+1}\right)}{k-n_{p+q+1}}\right] \circ \mathcal{F}_{k}^{*}, \\
\tilde{b}\left(\Pi_{n^{\prime \prime}} U^{\prime \prime} ; k, n_{p+q+1}\right) & =\mathcal{F}_{k} \circ\left[b\left(\Pi_{n^{\prime \prime}} U^{\prime \prime}, n_{p+q+1}\right)\right] \circ \mathcal{F}_{n_{p+q+1}}^{*}\left(k-n_{p+q+1}\right) .
\end{aligned}
$$

We used in the definition of $I$ and $I I$ that $a$ is scalar, so that in (3.2.37) it is meaningful to make $a\left(\Pi_{n_{n^{\prime}}} U^{\prime}, n_{p+q+1}\right)$ act on an element of $E_{k}$, as remarked before the statement of Proposition 3.2.7.

\section{Study of term $I$.}

We further decompose $I=I^{\prime}+I^{\prime \prime}$, where

$$
\begin{aligned}
I^{\prime}=\sum_{n_{0}} \cdots \sum_{n_{p+q+1}} & \chi_{p+q+1}\left(n_{0}, n^{\prime}, n^{\prime \prime}, n_{p+q+1}\right) \\
& \times \Pi_{n_{0}}\left[a\left(\Pi_{n^{\prime}} U^{\prime}, n_{p+q+1}\right) b\left(\Pi_{n^{\prime \prime}} U^{\prime \prime}, n_{p+q+1}\right) \Pi_{n_{p+q+1}} u_{p+q+1}\right] .
\end{aligned}
$$

Remark that $I^{\prime}$ is nothing but the first term on the right hand side of (3.2.34). Let us show that $I^{\prime \prime}$ is a remainder operator. We have

$$
\begin{aligned}
I^{\prime \prime}=\sum_{n_{0}} \cdots \sum_{n_{p+q+1}} \sum_{k}\left[\chi_{q+1}\left(n_{0}, n^{\prime}, k\right) \chi_{p+1}\left(k, n^{\prime \prime}, n_{p+q+1}\right)\right. & \left.\quad-\chi_{p+q+1}\left(n_{0}, n^{\prime}, n^{\prime \prime}, n_{p+q+1}\right)\right] \\
& \times \Pi_{n_{0}}\left[a\left(\Pi_{n^{\prime}} U^{\prime}, n_{p+q+1}\right) \Pi_{k}\left[b\left(\Pi_{n^{\prime \prime}} U^{\prime \prime}, n_{p+q+1}\right) \Pi_{n_{p+q+1}} u_{p+q+1}\right]\right] .
\end{aligned}
$$

The first cut-off in the above expression is supported in a domain of the form

$$
\begin{aligned}
& \left|n_{0}-k\right|<\delta\left(n_{0}+k\right), \quad\left|k-n_{p+q+1}\right|<\delta\left(k+n_{p+q+1}\right), \\
& \left|n^{\prime}\right|<\delta\left(n_{0}+k\right), \quad\left|n^{\prime \prime}\right|<\delta\left(k+n_{p+q+1}\right)
\end{aligned}
$$


and is equal to one on a domain of the same type. The second cut-off is supported inside a domain

$$
\left|n_{0}-n_{p+q+1}\right|<\delta\left(n_{0}+n_{p+q+1}\right), \quad \max \left(\left|n^{\prime}\right|,\left|n^{\prime \prime}\right|\right)<\delta\left(n_{0}+n_{p+q+1}\right)
$$

and is equal to 1 on a similar domain. By formula (3.2.10) of Corollary 3.2.4, the general term of (3.2.40) has $\mathcal{L}\left(L^{2}, L^{2}\right)$-norm bounded from above by

$$
C n_{p+q+1}^{d} \frac{\left|n^{\prime}\right|^{\nu+N}}{\left(\left|n_{0}-k\right|+\left|n^{\prime}\right|\right)^{N}} n_{p+q+1}^{d^{\prime}} \frac{\left|n^{\prime \prime}\right|^{\nu+N}}{\left(\left|k-n_{p+q+1}\right|+\left|n^{\prime \prime}\right|\right)^{N}} .
$$

Remark moreover that by (3.2.41), (3.2.42), $n_{0} \sim n_{p+q+1} \gg \max \left(\left|n^{\prime}\right|,\left|n^{\prime \prime}\right|\right)$ and if

$$
\left|n_{0}-k\right|+\left|k-n_{p+q+1}\right|+\left|n^{\prime}\right|+\left|n^{\prime \prime}\right|<\delta^{\prime}\left(n_{0}+n_{p+q+1}\right),
$$

for small enough $\delta^{\prime}>0$, both cut-offs in (3.2.40) equal one. Consequently, on the support, we may always extract from one of the factors of (3.2.43) a term decaying like $\left(n_{0}+n_{p+q+1}\right)^{-N}$. This shows that we get for $I^{\prime \prime}$ remainder type estimates of the form (2.1.15) with $d$ replaced by $d+d^{\prime}$.

Study of term $I I$.

We shall show that $I I$ gives the term $\operatorname{Op}(e(U ; \cdot)) u_{p+q+1}$ in (3.2.34). We shall need the following technical lemma:

Lemma 3.2.8. Let $d \in \mathbb{R}$ and $f: \mathbb{Z} \rightarrow \mathbb{C}$ be a function satisfying $\left|\partial_{n}^{\alpha} f(n)\right| \leq$ $C_{\alpha} n^{d-\alpha}$ for any $\alpha \in \mathbb{N}$. Define for $a, b \in \mathbb{Z}, a \neq b, g(a, b)=\frac{f(b)-f(a)}{b-a}$. Then one may extend $g$ to the diagonal $a=b$ and on the domain $|a-b| \leq \frac{1}{2}|a+b|$ one has the estimate

$$
\left|\partial_{a}^{\alpha} \partial_{b}^{\beta} g(a, b)\right| \leq C_{\alpha, \beta}(a+b)^{d-1-\alpha-\beta}
$$

for any $\alpha, \beta \in \mathbb{N}$.

Proof. Let us construct first $\chi \in \mathcal{S}(\mathbb{R})$ real-valued such that $\chi(0)=1, \chi(n)=$ $0 \forall n \in \mathbb{Z}^{*}$ and, for any $k \in \mathbb{N}$, there is $\chi_{k} \in \mathcal{S}(\mathbb{R})$ with

$$
\forall x \in \mathbb{R}, \chi^{(k)}(x)=\partial^{k} \chi_{k}(x),
$$

where we denote $\partial \chi(x)=\chi(x+1)-\chi(x)$ (extending the notation (1.2.3) to real arguments). First take $\gamma \in C_{0}^{\infty}(]-1,1[, \mathbb{R})$ with $\gamma(0)=1, \theta \in C_{0}^{\infty}(]-\pi, \pi[, \mathbb{R})$ even, such that $\sum_{k \in \mathbb{Z}} \theta(\xi-2 \pi k) \equiv 1$. Define $\chi$ by $\hat{\chi}(\xi)=\theta(\xi) \sum_{k=-\infty}^{+\infty} \hat{\gamma}(\xi+2 k \pi)$. Then, for $n \in \mathbb{Z}$,

$$
\chi(n)=\frac{1}{2 \pi} \int e^{i n \xi} \theta(\xi)\left(\sum_{k=-\infty}^{+\infty} \hat{\gamma}(\xi+2 k \pi)\right) d \xi=\gamma(n) .
$$

Moreover

$$
\chi^{\prime}(x)=\frac{1}{2 \pi} \int e^{i x \xi}\left(e^{i \xi}-1\right) \hat{\chi}_{1}(\xi) d \xi=\chi_{1}(x+1)-\chi_{1}(x)
$$

if we define $\hat{\chi}_{1}(\xi)=\frac{i \xi}{e^{i \xi}-1} \hat{\chi}(\xi)$, which belongs to $\mathcal{S}(\mathbb{R})$ by construction of $\hat{\chi}$. We deduce (3.2.46) from this equality by induction.

Now write, denoting by $\langle\cdot, \cdot\rangle$ the scalar product $\left\langle f_{1}, f_{2}\right\rangle=\sum_{-\infty}^{+\infty} f_{1}(n) \overline{f_{2}(n)}$,

$$
g(a, b)=\frac{1}{b-a} \sum_{n=-\infty}^{+\infty} f(n)(\chi(n-b)-\chi(n-a))=\langle f, H(\cdot, a, b)\rangle,
$$


where

$$
H(n, a, b)=-\int_{0}^{1} \chi^{\prime}(n-(1-t) b-t a) d t .
$$

This defines an extension of $g(a, b)$ to $a=b$. If we make the finite difference operator $\partial_{b}$ act on $H(n, a, b)$, we get

$$
\begin{aligned}
& \partial_{b}^{\beta} H(n, a, b) \\
& =-\int_{0}^{1} \cdots \int_{0}^{1} \chi^{(\beta+1)}\left(n-(1-t) b-t a-\left(s_{1}+\cdots+s_{\beta}\right)(1-t)\right)(t-1)^{\beta} d s_{1} \ldots d s_{\beta} d t .
\end{aligned}
$$

Using (3.2.46) on the right hand side, we see that we may write

$$
\partial_{b}^{\beta} H(n, a, b)=\partial_{n}^{\beta+1} H_{\beta}(n, a, b),
$$

where $H_{\beta}$ satisfies for any $N \in \mathbb{N}$ an estimate

$$
\left|H_{\beta}(n, a, b)\right| \leq C_{N} \int_{0}^{1}\langle n-(1-t) b-t a\rangle^{-N} d t .
$$

Consequently, if we write

$$
\partial_{b}^{\beta} g(a, b)=\left\langle f, \partial_{b}^{\beta} H(n, a, b)\right\rangle=\left\langle\left(\partial_{n}^{*}\right)^{\beta+1} f, H_{\beta}(n, a, b)\right\rangle
$$

and use the above upper bound, and the assumption $|a-b| \leq \frac{1}{2}|a+b|$, we obtain $\left|\partial_{b}^{\beta} g(a, b)\right| \leq C|a+b|^{d-1-\beta}$. One treats in the same way the action of difference operators acting on the first variable of $g$.

End of proof of Proposition 3.2.7. Denote by $\left(X_{1}, \ldots, X_{q}, n\right) \rightarrow A\left(X_{1}, \ldots, X_{q}, n\right)$ the function on $G \times \cdots \times G \times \mathbb{N}_{\tau}$ in terms of which the symbol $a\left(u_{1}, \ldots, u_{q}, n\right)$ is defined according to Definition 3.2.6 (see formula (3.2.32) ). Set

$$
A_{1}\left(X_{1}, \ldots, X_{q}, n_{p+q+1}, k\right)=\frac{A\left(X_{1}, \ldots, X_{q}, n_{p+q+1}\right)-A\left(X_{1}, \ldots, X_{q}, k\right)}{n_{p+q+1}-k}
$$

(taking by convention the quotient to be the extension of Lemma 3.2.8 when $n_{p+q+1}$ $=k$ ). By Lemma 3.2.8, $A_{1}$ satisfies, when $\left|n_{p+q+1}-k\right| \leq \frac{1}{2}\left(n_{p+q+1}+k\right)$ and $n_{p+q+1} \sim k$ is large enough,

$$
\left|\partial_{n_{p+q+1}}^{\alpha_{1}} \partial_{k}^{\alpha_{2}} A_{1}\left(X_{1}, \ldots, X_{q}, n_{p+q+1}, k\right)\right| \leq C_{\alpha \beta}\left(k+n_{p+q+1}\right)^{d-1-\alpha_{1}-\alpha_{2}} \prod_{j=1}^{q}\left|X_{j}\right|_{G} .
$$

In other words, assumption (3.2.11) of Corollary 3.2 .4 holds true. We denote by $\tilde{a}_{\chi}$ the product of $\tilde{a}$ given by (3.2.39) with $\chi_{q+1}\left(n_{0}, n^{\prime}, k\right)$, and by $\tilde{b}_{\chi}$ the product of $\tilde{b}$ by $\chi_{p+1}\left(k, n^{\prime \prime}, n_{p+q+1}\right)$. By (3.2.12),

$$
\begin{array}{r}
\left\|\partial_{n_{0}}^{\alpha}\left(\partial_{k}^{*}\right)^{\beta_{1}}\left(\partial_{n_{p+q+1}}^{*}\right)^{\beta_{2}}\left(\partial_{n_{0}}-\partial_{k}^{*}-\partial_{n_{p+q+1}}^{*}\right)^{\gamma} \tilde{a}_{\chi}\left(\Pi_{n^{\prime}} U^{\prime}, n_{p+q+1} ; n_{0}, k\right)\right\| \\
\leq C\left(n_{0}+k\right)^{d-\beta_{2}-\gamma-1} \frac{\left|n^{\prime}\right|^{\nu+N+\alpha+\beta_{1}+\beta_{2}+\gamma}}{\left(\left|n_{0}-k\right|+\left|n^{\prime}\right|\right)^{N}} \prod_{1}^{q}\left\|u_{j}\right\|_{L^{2}} .
\end{array}
$$

Moreover, by Proposition 3.2 .3 and the Leibniz formulas (1.2.6), (1.2.7), $\tilde{b}_{\chi} \in \Sigma_{p, 1}^{d^{\prime}, \nu}$ for some $\nu$. Now define

$$
e\left(U ; n_{0}, n_{p+q+1}\right)=\sum_{n^{\prime}} \sum_{n^{\prime \prime}} \sum_{k} \tilde{a}_{\chi}\left(\Pi_{n^{\prime}} U^{\prime}, n_{p+q+1} ; n_{0}, k\right) \tilde{b}_{\chi}\left(\Pi_{n^{\prime \prime}} U^{\prime \prime} ; k, n_{p+q+1}\right) .
$$


By the second Leibniz formula (1.2.7),

$$
\begin{aligned}
\left(\partial_{n_{0}}-\partial_{n_{p+q+1}}^{*}\right) e\left(\Pi_{n^{\prime}} U^{\prime}, \Pi_{n^{\prime \prime}} U^{\prime \prime} ; n_{0}, n_{p+q+1}\right) & =\sum_{k}\left(\left(\partial_{n_{0}}-\partial_{n_{p+q+1}}^{*}-\partial_{k}^{*}\right) \tilde{a}_{\chi}\right) \tilde{b}_{\chi} \\
& +\sum_{k} \tilde{a}_{\chi}\left(\partial_{k}-\partial_{n_{p+q+1}}^{*}\right) \tilde{b}_{\chi} \\
& -\sum_{k}\left(\partial_{n_{p+q+1}}^{*} \tilde{a}_{\chi}\right)\left(\partial_{n_{p+q+1}}^{*} \tilde{b}_{\chi}\right) .
\end{aligned}
$$

Using (3.2.47), and the fact that $\tilde{b}_{\chi}$ obeys symbol estimates of type (2.1.12), we see that the action of $\partial_{n_{0}}-\partial_{n_{p+q+1}}^{*}$ on $e$ gains one unit either on the order of $\tilde{a}_{\chi}$ or of $\tilde{b}_{\chi}$ in (3.2.49), losing a power of $\left|n^{\prime}\right|$ or $\left|n^{\prime \prime}\right|$. In the same way, one sees that a $\partial_{n_{0}}$ or a $\partial_{n_{p+q+1}}^{*}$ derivative does not change the order. Consequently, to check that $e \in \Sigma_{p+q, 1}^{d+d^{\prime}-1, \nu}$, we just have to check that (3.2.48) satisfies property (i) of Definition 2.1.1, and estimate (2.1.12) when $\alpha=\beta=\gamma=0$.

Since inequalities (3.2.41) are valid on the supports of $\tilde{a}_{\chi}, \tilde{b}_{\chi}$, (i) of Definition 2.1.1 holds true (if $\delta>0$ in (3.2.41) is small enough). Moreover, by (3.2.47) and the fact that $\tilde{b}_{\chi} \in \Sigma_{p, 1}^{d^{\prime}, \nu}$, we get for $\left\|e\left(\Pi_{n^{\prime}} U^{\prime}, \Pi_{n^{\prime \prime}} U^{\prime \prime} ; n_{0}, n_{p+q+1}\right)\right\|$ an upper bound given by

$$
\sum_{k}\left(n_{0}+k\right)^{d-1} \frac{\mu\left(n_{0}, n^{\prime}, k\right)^{\nu+N_{1}}}{S\left(n_{0}, n^{\prime}, k\right)^{N_{1}}}\left(k+n_{p+q+1}\right)^{d^{\prime}} \frac{\mu\left(k, n^{\prime \prime}, n_{p+q+1}\right)^{\nu+N_{2}}}{S\left(k, n^{\prime \prime}, n_{p+q+1}\right)^{N_{2}}} \prod_{1}^{p+q}\left\|u_{j}\right\|_{L^{2}} .
$$

Since on the support we have $k \sim n_{0} \sim n_{p+q+1}$, we may use Lemma 2.2.5 to get the upper bound (for new values of $\nu, N$ )

$$
C\left(n_{0}+n_{p+q+1}\right)^{d+d^{\prime}-1} \frac{\mu\left(n_{0}, n^{\prime}, n^{\prime \prime}, n_{p+q+1}\right)^{\nu+N}}{S\left(n_{0}, n^{\prime}, n^{\prime \prime}, n_{p+q+1}\right)^{N}} \prod_{1}^{p+q}\left\|u_{j}\right\|_{L^{2}},
$$

which is the wanted estimate.

(ii) We have, using the notation (2.2.1), (3.2.5) and the fact that $\chi$ is even,

$$
\begin{aligned}
& a_{\chi}^{\bullet}\left(U^{\prime} ; n_{0}, n_{q+1}\right)- a_{\chi}\left(U^{\prime} ; n_{0}, n_{q+1}\right) \\
&=\sum_{n_{1}} \cdots \sum_{n_{p}} \chi\left(\frac{\left|n^{\prime}\right|}{n_{0}+n_{q+1}}\right) \chi\left(\frac{n_{0}-n_{q+1}}{n_{0}+n_{q+1}}\right) \\
& \quad \times \mathcal{F}_{n_{0}} \circ\left[a\left(\Pi_{n^{\prime}} U^{\prime}, n_{0}\right)-a\left(\Pi_{n^{\prime}} U^{\prime}, n_{q+1}\right)\right] \circ \mathcal{F}_{n_{q+1}}^{*} .
\end{aligned}
$$

One has just to apply the proof of Proposition 3.2 .3 together with estimate (3.2.45) to check that the above formula defines an element of $\Sigma_{q, 1}^{d-1, \nu}$.

3.3. Polyhomogenous symbols. We collect in this subsection corollaries of the results obtained in subsections 3.1 and 3.2, which apply to symbols which are not necessarily multilinear in the arguments $u_{1}, \ldots, u_{p}$.

Definition 3.3.1. (i) For $d \in \mathbb{R}, \nu \in \mathbb{R}_{+}, N_{0} \in \mathbb{N}^{*}$, we denote by $\widetilde{\Sigma}_{N_{0}}^{d, \nu}$ the space of functions $b: \mathcal{E} \times \mathbb{N}_{\tau} \times \mathbb{N}_{\tau} \rightarrow \mathcal{L}\left(\ell^{2}, \ell^{2}\right)$ such that there is a finite family $\left(b_{p}\right)_{p=0, \ldots, P}$ 
of elements $b_{p} \in \Sigma_{p, N_{0}}^{d, \nu}$ with

$$
b\left(u ; n_{0}, n_{p+1}\right)=\sum_{p=0}^{P} b_{p}(\underbrace{u, \ldots, u}_{p \text { times }} ; n_{0}, n_{p+1})
$$

for any $n_{0}, n_{p+1} \in \mathbb{N}_{\tau}, u \in \mathcal{E}$.

(ii) For $d \in \mathbb{N}$, we denote by $\widetilde{S}^{d}$ the space of functions $b: \mathcal{E} \times \mathbb{N}_{\tau} \rightarrow \mathcal{L}\left(\mathcal{E}, L^{2}\right)$ such that there is a finite family $\left(b_{p}\right)_{p=0, \ldots, P}$ of elements $b_{p} \in S_{p}^{d}$ with

$$
b(u, n)=\sum_{p=0}^{P} b_{p}(\underbrace{u, \ldots, u}_{p \text { times }}, n)
$$

for any $n \in \mathbb{N}_{\tau}, u \in \mathcal{E}$. We define in a similar way $\widetilde{S}_{\mathrm{sc}}^{d}$ from $S_{p, \mathrm{sc}}^{d}$.

(iii) For $\nu \in \mathbb{R}_{+}, d \in \mathbb{R}$, we denote by $\widetilde{\mathcal{R}}^{d, \nu}$ the space of all maps $M: \mathcal{E} \rightarrow L^{2}$ such that there is a finite family of maps $M_{p} \in \mathcal{R}_{p}^{d, \nu} p=1, \ldots, P$ with

$$
M(u)=\sum_{p=1}^{P} M_{p}(\underbrace{u, \ldots, u}_{p \text { times }})
$$

for any $u \in \mathcal{E}$. Sometimes, we shall use the same notation for maps $(u, v) \rightarrow M(u, v)$ depending on two arguments $u, v \in \mathcal{E}$, and which may be written as a sum of multilinear expressions of the form $M_{p}(u, \ldots, u, v \ldots, v)$ where the total number of arguments is $p$ and $1 \leq p \leq P$.

We define the valuation $v(b)$ of a symbol $b$ (resp. $v(M)$ of an element $M$ of $\widetilde{\mathcal{R}}^{d, \nu}$ ) as the smallest $p \geq 0$ (resp. $p \geq 1$ ) such that $b_{p} \not \equiv 0$ in (3.3.1), (3.3.2) (resp. $M_{p} \not \equiv 0$ in (3.3.3) $)$. The modified valuation $v^{\prime}(b)$ of a symbol is the smallest $p \geq 1$ such that $b_{p} \not \equiv 0$ in (3.3.1), (3.3.2).

In section 4 below, we shall have to use symbols verifying conditions of type (1.1.3). We introduce the following definition.

Definition 3.3.2. Let $\kappa$ be an odd integer, $r \in \mathbb{N}$ with $\kappa \leq r-1 \leq 2 \kappa$. We say that a symbol $b \in \widetilde{\Sigma}_{N_{0}}^{d, \nu}$ (resp. $b \in \widetilde{S}^{d}$, resp. an operator $M \in \widetilde{\mathcal{R}}^{d, \nu}$ ) satisfies condition $C(\kappa, r)$ if and only if $b=b_{0}+\sum_{p=\kappa}^{\kappa_{1}} b_{p}$ (resp. $M=\sum_{p=\kappa}^{\kappa_{1}} M_{p+1}$ ) with $b_{p} \in \Sigma_{p, N_{0}}^{d, \nu}$ (resp. $b_{p} \in S_{p}^{d}$, resp. $M_{p+1} \in \mathcal{R}_{p+1}^{d, \nu}$ ) and $b_{p} \equiv 0$ (resp. $M_{p+1} \equiv 0$ ) when $p$ is an even integer $2 k$ satisfying $\kappa \leq 2 k<r-1$.

We shall use below several times the following remark. Let $L$ be a linear map (resp. $B$ be a bilinear map) from one (resp. the product of two) of the above spaces of symbols or operators to a third space of that type. Assume that $L$ (resp. B) respects the natural graduations of these spaces. Then $L$ (resp. $B$ ) sends symbols or operators satisfying $C(\kappa, r)$ to symbols or operators satisfying $C(\kappa, r)$.

This is trivial for linear maps. In the bilinear case, this follows from the fact that in an expression of the form $B(a, b)$, the contributions of type $B\left(a_{q}, b_{p}\right)$ with $q>0$ and $p>0$ are homogeneous of degree $p+q \geq 2 \kappa \geq r-1$ (since $v^{\prime}(a) \geq \kappa$, $\left.v^{\prime}(b) \geq \kappa\right)$, so the condition imposed by $C(\kappa, r)$ on $B\left(a_{q}, b_{p}\right)$ is void. Only terms of type $B\left(a_{0}, b_{p}\right), B\left(a_{q}, b_{0}\right)$ have to be taken into consideration, and they satisfy the condition of the definition. 
We extend the definition of the quantization of operators by linearity, setting for $b \in \widetilde{\Sigma}_{N_{0}}^{d, \nu}$ or $b \in \widetilde{S}^{d}$, respectively,

$$
\begin{aligned}
& \mathrm{Op}(b(u ; \cdot))=\sum_{p=0}^{P} \mathrm{Op}\left(b_{p}(u, \ldots, u ; \cdot)\right), \\
& \widetilde{\mathrm{Op}}(b(u, \cdot))=\sum_{p=0}^{P} \widetilde{\mathrm{Op}}\left(b_{p}(u, \ldots, u, \cdot)\right) .
\end{aligned}
$$

By Proposition 2.1.3 and Lemma 2.1.7 maps such as $(u, v) \rightarrow \mathrm{Op}(b(u ; \cdot)) v$, for $b \in$ $\widetilde{\Sigma}_{N_{0}}^{d, \nu}$ (resp. $u \rightarrow R(u)$ for $\left.R \in \widetilde{\mathcal{R}}^{d, \nu}\right)$ extend from $\mathcal{E} \times \mathcal{E}(\operatorname{resp} . \mathcal{E})$ to $H^{s}\left(\mathbb{S}^{1}, G \otimes \mathbb{K}\right)^{2}$ (resp. $H^{s}\left(\mathbb{S}^{1}, G \otimes \mathbb{K}\right)$ ) if $s$ is large enough. We use this in the following corollaries, which are stated for arguments $u, v$ smooth enough, but need only to be checked when $u, v \in \mathcal{E}$ by density.

Corollary 3.3.3. Let $P \in \mathbb{N}^{*}$ be given. There is $\nu \in \mathbb{R}_{+}$such that if we define for $d \in \mathbb{R}, b \in \widetilde{S}^{d}, \chi \in C_{0}^{\infty}(\mathbb{R}), \chi \equiv 1$ close to zero, Supp $\chi$ small enough, $b_{\chi}=$ $\sum_{p=0}^{P} b_{p, \chi} \in \widetilde{\Sigma}_{1}^{d, \nu}$, we may find a symbol $b^{0} \in \widetilde{\Sigma}_{1}^{0, \nu+d_{+}}$and an operator $M \in \widetilde{\mathcal{R}}^{0, \nu+d_{+}}$ such that for any smooth enough $u$,

$$
\widetilde{\mathrm{Op}}(b(u, \cdot)) u=\mathrm{Op}\left(b_{\chi}(u ; \cdot)\right) u+\mathrm{Op}\left(b^{0}(u ; \cdot)\right) u+M(u) .
$$

Moreover, one has

$$
v\left(b_{\chi}\right) \geq v(b), v\left(b^{0}\right) \geq v^{\prime}(b), v(M) \geq v^{\prime}(b)+1
$$

and $b_{\chi}, b^{0}, M$ satisfy condition $C(\kappa, r)$ if $b$ does so.

Proof. We decompose $b=\sum_{p=0}^{P} b_{p}$ and apply Proposition 3.2.5 to each component. We obtain (3.3.5) and (3.3.6), remembering that for $p=0, b_{0}$ does not depend on $u$, so that $\widetilde{\mathrm{Op}}\left(b_{0}\right)=\mathrm{Op}\left(b_{0, \chi}\right)$ for any $\chi$ as in the statement of the theorem. Consequently $b_{0}$ does not contribute to the last two terms in (3.3.5), which implies the last two inequalities in (3.3.6).

Corollary 3.3.4. Let $d, d^{\prime} \in \mathbb{R}, a \in \widetilde{S}_{\mathrm{sc}}^{d}, b \in \widetilde{S}_{\mathrm{sc}}^{d^{\prime}}$. Let $\chi \in C_{0}^{\infty}(\mathbb{R}), \chi \equiv 1$ close to zero, with small enough support. There are $\nu \in \mathbb{R}_{+}$independent of $d, d^{\prime}$, a symbol $e \in \widetilde{\Sigma}_{1}^{d+d^{\prime}-1, \nu}$ and a remainder operator $M \in \widetilde{\mathcal{R}}^{d+d^{\prime}, \nu} \subset \widetilde{\mathcal{R}}^{0, \nu+d_{+}+d_{+}^{\prime}}$ such that for any smooth enough $u, v$,

$$
\mathrm{Op}\left(a_{\chi}(u ; \cdot)\right) \circ \mathrm{Op}\left(b_{\chi}(u ; \cdot)\right) v=\mathrm{Op}\left((a \circ b)_{\chi}(u ; \cdot)\right) v+\mathrm{Op}(e(u ; \cdot)) v+M(u, v) .
$$

Moreover

$$
v(e) \geq \min \left(v^{\prime}(a), v^{\prime}(b)\right), v(M) \geq \min \left(v^{\prime}(a), v^{\prime}(b)\right)+1 .
$$

If $v(a)=v^{\prime}(a)>0, v(b)=v^{\prime}(b)>0$, we have

$$
v(e) \geq v^{\prime}(a)+v^{\prime}(b), v(M) \geq v^{\prime}(a)+v^{\prime}(b)+1 .
$$

Moreover $a \circ b$, $e$ and $M$ satisfy $C(\kappa, r)$ if $a$ and $b$ do so.

Proof. We decompose $a=\sum_{q=0}^{Q} a_{q}, b=\sum_{p=0}^{P} b_{p}$ and apply Proposition 3.2.7 to each contribution, remarking that $\mathrm{Op}\left(a_{0, \chi}\right) \mathrm{Op}\left(b_{0, \chi}\right)=\mathrm{Op}\left(\left(a_{0} \circ b_{0}\right)_{\chi}\right)$, so that all contributions to $e$ and $M$ come from compositions with $p>0$ or $q>0$. The last statement comes from the remark after Definition 3.3.2. 
Corollary 3.3.5. (i) Let $\nu \in \mathbb{R}_{+}, N_{0} \in \mathbb{N}^{*}$. There is $\nu^{\prime} \in \mathbb{R}_{+}$, and for any $d, d^{\prime} \in \mathbb{R}$, any $a \in \widetilde{\Sigma}_{N_{0}}^{d, \nu}, b \in \widetilde{\Sigma}_{N_{0}}^{d^{\prime}, \nu}$ satisfying condition $(i)_{\delta}$ of Definition 2.1.1 with small enough $\delta>0$, there is a symbol $a \# b \in \widetilde{\Sigma}_{N_{0}}^{d+d^{\prime}, \nu^{\prime}}$ such that for any smooth enough $u$,

$$
\mathrm{Op}(a(u ; \cdot)) \circ \mathrm{Op}(b(u ; \cdot)) u=\mathrm{Op}(a \# b(u ; \cdot)) u .
$$

Moreover $v(a \# b) \geq v(a)+v(b)$, and $a \# b$ satisfies $C(\kappa, r)$ if $a, b$ do so.

(ii) Assume moreover that the homogeneous components $a_{q}\left(u ; n_{0}, n_{q+1}\right)$ and $b_{p}\left(u ; n_{0}^{\prime}, n_{p+1}^{\prime}\right)$ of a, $b$ commute for large enough $n_{0}, n_{q+1}, n_{0}^{\prime}, n_{p+1}^{\prime}$ and that $a_{0} b_{0} \equiv$ $b_{0} a_{0}$. There is $c \in \widetilde{\Sigma}_{N_{0}}^{d+d^{\prime}-1, \nu^{\prime}}$ such that

$$
[\mathrm{Op}(a(u ; \cdot)), \mathrm{Op}(b(u ; \cdot))] u=\mathrm{Op}(c(u ; \cdot)) u
$$

for any smooth enough $u$, and $v(c) \geq \min \left(v^{\prime}(a), v^{\prime}(b)\right)$. If moreover $v(a)=v^{\prime}(a)>0$ and $v(b)=v^{\prime}(b)>0$, then $v(c) \geq v^{\prime}(a)+v^{\prime}(b)$. Finally if $a, b$ satisfy $C(\kappa, r)$, the same holds true for $c$.

Proof. We decompose again $a=\sum_{p=0}^{P} a_{p}, b=\sum_{q=0}^{Q} b_{q}$ and define $a \# b$ or $c$ using the linearity in (i), (ii) of Proposition 2.2.2, The statement concerning valuations in (ii) of the corollary comes from the fact that $\left[\mathrm{Op}\left(a_{0}\right), \mathrm{Op}\left(b_{0}\right)\right]=0$ since these operators are constant coefficient ones.

Corollary 3.3.6. (i) Let $d, d^{\prime} \in \mathbb{R}, p \in \mathbb{N}^{*}, \nu \in \mathbb{R}_{+}, N_{0} \in \mathbb{N}^{*}$. Let $a \in \Sigma_{p, N_{0}}^{d, \nu}$, $b \in \widetilde{\Sigma}_{N_{0}}^{d^{\prime}, \nu}$, and assume that they satisfy condition (i) of Definition 2.1.1 with a small enough $\delta>0$. Then there are $\nu^{\prime}=2 \nu+d_{+}^{\prime}+1$ and $c \in \widetilde{\Sigma}_{N_{0}}^{d, \nu^{\prime}}$ with

$$
\mathrm{Op}[a[\mathrm{Op}(b(u ; \cdot)) u, \underbrace{u, \ldots, u}_{p-1 \text { times }} ; \cdot]] v=\operatorname{Op}(c(u ; \cdot)) v
$$

for any smooth enough $u, v$. Moreover $v(c) \geq p+v(b)$ and $c$ satisfies $C(\kappa, r)$ if $b$ does so and $p$ is odd, $p \geq \kappa$.

(ii) Let $d \in \mathbb{R}, d^{\prime} \in \mathbb{R}, \nu \in \mathbb{R}_{+}, p \in \mathbb{N}^{*}, N_{0} \in \mathbb{N}^{*}, a \in \Sigma_{p, N_{0}}^{d, \nu}$ satisfying condition (i) $\delta$ of Definition 2.1 .1 with a small enough $\delta>0$. Let $M \in \widetilde{\mathcal{R}}^{d^{\prime}, \nu}$. There are $\nu^{\prime}=d_{+}^{\prime}+2 \nu+1, \nu^{\prime \prime}=2 \nu+1, b \in \widetilde{\Sigma}_{N_{0}}^{d, \nu^{\prime}}$ and $R \in \widetilde{\mathcal{R}}^{d+d_{+}^{\prime}, \nu^{\prime \prime}} \subset \widetilde{\mathcal{R}}^{0, \nu^{\prime \prime}+d_{+}+d_{+}^{\prime}}$ such that for any smooth enough $u, v$,

$$
\mathrm{Op}(a(M(u), \underbrace{u, \ldots, u}_{p-1} ; \cdot)) v=\mathrm{Op}(b(u ; \cdot)) v+R(u, v)
$$

with $v(b) \geq v(M)+p-1, v(R) \geq v(M)+p$. Moreover $b, R$ satisfy $C(\kappa, r)$ if $M$ does so and $p$ is odd, $p \geq \kappa$.

(iii) Let $d \in \mathbb{R}, d^{\prime} \in \mathbb{R}, \nu, \nu^{\prime} \in \mathbb{R}_{+}, N_{0} \in \mathbb{N}^{*}, a \in \widetilde{\Sigma}_{N_{0}}^{d, \nu}, M \in \widetilde{\mathcal{R}}^{d^{\prime}, \nu}$. There is $\nu^{\prime \prime}=\nu+\nu^{\prime}+1$ such that $u \rightarrow R(u)=\operatorname{Op}(a(u ; \cdot)) M(u)$ is in $\widetilde{\mathcal{R}}^{d+d^{\prime}, \nu^{\prime \prime}}$ and $v(R) \geq v(a)+v(M)$. Moreover $R$ satisfies $C(\kappa, r)$ if $a$ and $M$ do so.

These statements follow from Propositions 2.2.3 2.2.4 and 2.2.6. In the same way, we deduce from Proposition 2.2.7

Corollary 3.3.7. (i) Let $d, d^{\prime} \in \mathbb{R}, \nu, \nu^{\prime} \in \mathbb{R}_{+}, N_{0} \in \mathbb{N}, q \in \mathbb{N}^{*}$. Let $a \in \widetilde{\Sigma}_{N_{0}}^{d, \nu}$ and $M \in \mathcal{R}_{q}^{d^{\prime}, \nu^{\prime}}$. There is $\nu^{\prime \prime}=d_{+}+\nu+\nu^{\prime}+1$ such that the operator $u \rightarrow R(u)$ given by $R(u)=M(\operatorname{Op}(a(u ; \cdot)) u, u, \ldots, u)$ is in $\widetilde{\mathcal{R}}^{d^{\prime}, \nu^{\prime \prime}}$ with $v(R) \geq q+v(a)$. Moreover $R$ satisfies $C(\kappa, r)$ if a does so and $q-1$ is an odd integer $q-1 \geq \kappa$. 
(ii) Let $M_{1} \in \mathcal{R}_{q}^{d, \nu}, M_{2} \in \widetilde{\mathcal{R}}^{d^{\prime}, \nu^{\prime}}$. Then there is $\nu^{\prime \prime}=\nu+\nu^{\prime}+d_{+}^{\prime}+1$ such that $R(u)=M_{1}\left(M_{2}(u), u, \ldots, u\right)$ is in $\widetilde{\mathcal{R}}^{d, \nu^{\prime \prime}}$ with $v(R) \geq v\left(M_{2}\right)+q-1$.

Let us conclude this subsection with the following technical lemma.

Lemma 3.3.8. (i) Let $a(\lambda)$ be a smooth function on $\mathbb{R}_{+}$satisfying for any $k$, $\left|\partial_{\lambda}^{k} a(\lambda)\right| \leq C_{k} \lambda^{1-k}$ when $\lambda \rightarrow+\infty$. Let $\left(n_{1}, \ldots, n_{p}\right)=n^{\prime} \rightarrow G\left(n^{\prime}\right)$ be a realvalued function defined on $\mathbb{N}_{\tau}^{p}$, such that there is $C>0$ with $\left|G\left(n^{\prime}\right)\right| \leq C\left(1+\left|n^{\prime}\right|\right)$. Consider the function

$$
F\left(n_{0}, n_{1}, \ldots, n_{p+1}\right)=a\left(n_{0}\right)-a\left(n_{p+1}\right)+G\left(n_{1}, \ldots, n_{p}\right)
$$

and assume that there is $c>0, N_{0} \in \mathbb{N}^{*}$ such that for any $n_{0}, n_{p+1} \in \mathbb{N}_{\tau}, n^{\prime} \in \mathbb{N}_{\tau}^{p}$ satisfying $\left|n_{0}-n_{p+1}\right| \leq \frac{1}{4}\left(n_{0}+n_{p+1}\right),\left|n^{\prime}\right| \leq \frac{1}{4}\left(n_{0}+n_{p+1}\right)$ one has

$$
\left|F\left(n_{0}, \ldots, n_{p+1}\right)\right| \geq c\left(1+\left|n_{0}-n_{p+1}\right|\right)\left|n^{\prime}\right|^{-N_{0}} .
$$

Then we have for any $\alpha, \beta, \gamma \in \mathbb{N}$, and any $\left(n_{0}, n^{\prime}, n_{p+1}\right) \in \mathbb{N}_{\tau}^{p+2}$ satisfying the preceding inequalities,

$$
\begin{aligned}
& \left|\partial_{n_{0}}^{\alpha}\left(\partial_{n_{p+1}}^{*}\right)^{\beta}\left(\partial_{n_{0}}-\partial_{n_{p+1}}^{*}\right)^{\gamma} \frac{1}{F\left(n_{0}, \ldots, n_{p+1}\right)}\right| \\
& \leq C_{\alpha \beta \gamma}\left(n_{0}+n_{p+1}\right)^{-\gamma}\left|n^{\prime}\right|^{N_{0}(\alpha+\beta+\gamma+1)}\left(1+\left|n_{0}-n_{p+1}\right|\right)^{-1} .
\end{aligned}
$$

(ii) If instead of (3.3.11), $F$ satisfies when $\left|n_{0}-n_{p+1}\right| \leq \frac{1}{4}\left(n_{0}+n_{p+1}\right),\left|n^{\prime}\right| \leq$ $\frac{1}{4}\left(n_{0}+n_{p+1}\right)$,

$$
\left|F\left(n_{0}, \ldots, n_{p+1}\right)\right| \geq c\left(n_{0}+n_{p+1}\right)\left|n^{\prime}\right|^{-N_{0}},
$$

then (3.3.12) holds true with the right hand side replaced by

$$
C_{\alpha \beta \gamma}\left(n_{0}+n_{p+1}\right)^{-1-\gamma}\left|n^{\prime}\right|^{N_{0}(\alpha+\beta+\gamma+1)} .
$$

Proof. (i) We may assume in (3.3.12) that $\alpha+\beta+\gamma>0$ since the inequality without derivatives follows from (3.3.11). Remark that we then have

$$
\left|\partial_{n_{0}}^{\alpha}\left(\partial_{n_{p+1}}^{*}\right)^{\beta}\left(\partial_{n_{0}}-\partial_{n_{p+1}}^{*}\right)^{\gamma} F\left(n_{0}, \ldots, n_{p+1}\right)\right| \leq C\left(1+\left|n_{0}-n_{p+1}\right|\right)\left(n_{0}+n_{p+1}\right)^{-\gamma} .
$$

This follows from Lemma 3.2 .8 applied to $g\left(n_{0}, n_{p+1}\right)=\frac{a\left(n_{0}\right)-a\left(n_{p+1}\right)}{n_{0}-n_{p+1}}$ and from the Leibniz formulas (1.2.6), (1.2.7). We shall show that for any $\alpha, \beta, \gamma$ we may write the quantity estimated on the left hand side of (3.3.12) as a linear combination, indexed by $k=1, \ldots, \alpha+\beta+\gamma$, of expressions of the form

$$
\frac{H_{k}}{F_{1} \cdots F_{k+1}}\left(n_{0}, \ldots, n_{p+1}\right)
$$

where each function $H_{k}$ satisfies

$$
\begin{aligned}
& \left|\partial_{n_{0}}^{\alpha^{\prime}}\left(\partial_{n_{p+1}}^{*}\right)^{\beta^{\prime}}\left(\partial_{n_{0}}-\partial_{n_{p+1}}^{*}\right)^{\gamma^{\prime}} H_{k}\left(n_{0}, \ldots, n_{p+1}\right)\right| \\
& \quad \leq C\left(1+\left|n_{0}-n_{p+1}\right|\right)^{k}\left(n_{0}+n_{p+1}\right)^{-\gamma-\gamma^{\prime}},
\end{aligned}
$$

and where $F_{1}, \ldots, F_{k+1}$ satisfy (3.3.11). Inequality (3.3.12) will then follow from (3.3.17) with $\alpha^{\prime}=\beta^{\prime}=\gamma^{\prime}=0$.

To obtain the structure (3.3.16), we just have to show that if we apply to (3.3.16) a derivative $\partial_{n_{0}}^{\alpha_{0}}\left(\partial_{n_{p+1}}^{*}\right)^{\beta_{0}}\left(\partial_{n_{0}}-\partial_{n_{p+1}}^{*}\right)^{\gamma_{0}}$ with $\alpha_{0}+\beta_{0}+\gamma_{0}=1$, we get the sum 
of an expression $\tilde{H}_{k}\left(F_{1} \cdots F_{k+1}\right)^{-1}$, where $\tilde{H}_{k}$ satisfies (3.3.17) with $\gamma$ replaced by $\gamma+\gamma_{0}$, and of a quantity $\tilde{H}_{k+1}\left(\tilde{F}_{1} \cdots \tilde{F}_{k+2}\right)^{-1}$, with $\tilde{H}_{k+1}$ satisfying (3.3.17) with $k$ replaced by $k+1$ and $\gamma$ by $\gamma+\gamma_{0}$, and with $\tilde{F}_{j}$ satisfying (3.3.11). This follows from the Leibniz formulas (1.2.6), (1.2.7) and from (3.3.15), (3.3.17). This concludes the proof.

(ii) The proof is the same, replacing in (3.3.15), (3.3.17) the $1+\left|n_{0}-n_{p+1}\right|$ factor by $n_{0}+n_{p+1}$.

\section{LONG-TIME EXISTENCE}

4.1. Strategy of proof. The aim of this section is to prove Theorem 1.1.1. Our strategy will be to combine the methods used by Bourgain [5], Bambusi 1], Bambusi and Grébert [3], Delort and Szeftel [10] for semi-linear equations, with the wellknown approach allowing one to obtain quasi-linear energy inequalities, namely diagonalization of the principal symbol of the equation.

Let us describe the steps that we shall follow, forgetting the necessary technicalities we shall have to introduce later on. We denote by $\Lambda_{m}=\sqrt{-\Delta+V+m^{2}}$, and we shall consider an equivalent system to the scalar equation for $u=\left[\begin{array}{c}\Lambda_{m} v \\ \partial_{t} v\end{array}\right]$, of type $\partial_{t} u=\widetilde{\mathrm{Op}}(M(u, \cdot)) u$, where $M$ will be a symbol of order 1 , belonging to the class introduced in subsection 3.1. We would like to control over long-time intervals the Sobolev energy of $u$,

$$
\left\langle\Lambda_{m}^{s} u(t, \cdot), \Lambda_{m}^{s} u(t, \cdot)\right\rangle
$$

If one computes the time derivative of this expression, one gets

$$
2 \operatorname{Re}\left\langle\Lambda_{m}^{s} \widetilde{\mathrm{Op}}(M(u, \cdot)) u, \Lambda_{m}^{s} u\right\rangle .
$$

If $M(u, \cdot)=M_{0}(\cdot)+M^{\kappa}(u, \cdot)$ is the sum of two anti-selfadjoint matrices, with $M_{0}$ independent of $u$ and $M^{\kappa}$ homogeneous of degree $\kappa>0$ in $u$, symbolic calculus shows that the above expression may be written as

$$
\langle\widetilde{\mathrm{Op}}(b(u, \cdot)) u, u\rangle
$$

where $b$ is a selfadjoint symbol of order $2 s$ vanishing at least at order $\kappa$ at $u=0$. Consequently, for $s$ large enough, this bracket is bounded from above by $C\|u\|_{H^{s}}^{\kappa+2}$, and one gets the estimate

$$
\frac{d}{d t}\|u(t, \cdot)\|_{H^{s}}^{2} \leq C\|u(t, \cdot)\|_{H^{s}}^{\kappa+2} .
$$

This is a way to recover the local existence result asserting that for smooth data of size $\epsilon \rightarrow 0$, the solution exists at least over an interval of time of length $c \epsilon^{-\kappa}$. Our goal here is to obtain a better result when $\kappa$ is odd (and when the parameter $m$ is outside a subset of zero measure). Namely we want to obtain a solution over a time interval of length $c \epsilon^{-2 \kappa}$. From (4.1.1) to (4.1.3) we know that

$$
\frac{d}{d t}\left\langle\Lambda_{m}^{s} u(t, \cdot), \Lambda_{m}^{s} u(t, \cdot)\right\rangle=\langle\widetilde{\mathrm{Op}}(b(u, \cdot)) u, u\rangle .
$$

We would like to add on the left hand side a new contribution, of the form $\langle\widetilde{\mathrm{Op}}(a(u, \cdot)) u, u\rangle$, vanishing at order $\kappa+2$ at 0 , with a symbol $a$ of order $2 s$, determined in such a way that the time derivative of this quantity will cancel out the right hand side of (4.1.5), up to remainders $O\left(\|u\|_{H^{s}}^{2 \kappa+2}\right)$. If we compute 
$\frac{d}{d t}\langle\widetilde{\mathrm{Op}}(a(u, \cdot)) u, u\rangle$ we get from the action of $d / d t$ on the $u$ 's that are not in the argument of $a$, a contribution of type

$$
\langle[\widetilde{\mathrm{Op}}(a(u, \cdot)) \widetilde{\mathrm{Op}}(M(u, \cdot))+\widetilde{\mathrm{Op}}(M(u, \cdot)) * \widetilde{\mathrm{Op}}(a(u, \cdot))] u, u\rangle .
$$

Remember that $M(u, \cdot)=M_{0}(\cdot)+M^{\kappa}(u, \cdot)$. Consider the expression obtained by replacing in (4.1.6) $M(u, \cdot)$ by $M^{\kappa}(u, \cdot)$ : we get a term homogeneous of degree $2 \kappa+2$ in $u$. In a semi-linear framework, i.e. when $M^{\kappa}$ is a symbol of order 0 , this gives a contribution to (4.1.6) which is $O\left(\|u\|_{H^{s}}^{2 \kappa+2}\right)$, since $a$ is of order $2 s$. In our quasi-linear framework, $M^{\kappa}(u, \cdot)$ is a symbol of order 1 , which a priori loses one extra derivative. The way to circumvent that difficulty is well known: one has to arrange so that $a$ will be selfadjoint and commute with $M^{\kappa}$. Then since $M^{\kappa}(u, \cdot)$ is assumed anti-selfadjoint, the contribution of $M^{\kappa}$ to (4.1.6) may be written in terms of a commutator $\left[\widetilde{\mathrm{Op}}(a(u, \cdot)), \widetilde{\mathrm{Op}}\left(M^{\kappa}(u, \cdot)\right)\right] u$. The symbolic calculus we studied in the preceding sections shows that this commutator gains one derivative, so that again the contribution of $M^{\kappa}$ to (4.1.6) is $O\left(\|u\|_{H^{s}}^{2 \kappa+2}\right)$. In other words, up to such nice remainders, $\frac{d}{d t}\langle\widetilde{\mathrm{Op}}(a(u, \cdot)) u, u\rangle$ will be given by contributions of type (4.1.6) with $M$ replaced by $M_{0}$, and by similar terms coming from the action of $\frac{d}{d t}$ on those $u$ inside the argument of $a$. The last step of the proof will be to show that we may choose $a$ so that these contributions to $\frac{d}{d t}\langle\widetilde{\mathrm{Op}}(a(u, \cdot)) u, u\rangle$ will cancel out the right hand side of 4.1.5).

To ensure the commutator property of $a$ with $M$, we start instead of (4.1.1) with

$$
\left\langle\Lambda_{m}^{s} \tilde{u}(t, \cdot), \Lambda_{m}^{s} \tilde{u}(t, \cdot)\right\rangle
$$

where $\tilde{u}$ is a new unknown defined in terms of $u$ by $\tilde{u}=Q(u) u, Q$ being a matrix such that $D(u, \cdot)=Q(u) M(u, \cdot) Q(u)^{-1}$ is diagonal. Computing the time derivative of (4.1.7), we shall get instead of (4.1.5) an expression

$$
\langle\widetilde{\mathrm{Op}}(b(u, \cdot)) \tilde{u}, \tilde{u}\rangle
$$

that we will try to cancel out by adding to (4.1.7) a quantity

$$
\langle\widetilde{\mathrm{Op}}(a(u, \cdot)) \tilde{u}, \tilde{u}\rangle,
$$

where $a$ is again a symbol to be determined. When we shall compute the time derivative of (4.1.9), the contribution corresponding to (4.1.6) will be

$$
\langle[\widetilde{\mathrm{Op}}(a(u, \cdot)) \widetilde{\mathrm{Op}}(D(u, \cdot))+\widetilde{\mathrm{Op}}(D(u, \cdot)) * \widetilde{\mathrm{Op}}(a(u, \cdot))] \tilde{u}, \tilde{u}\rangle .
$$

Now since $D$ is diagonal, and since we shall look for a diagonal symbol $a$, the commutation property between symbols, $a D=D a$, will hold true automatically. Moreover $a$ will be taken to be selfadjoint and $D$ will be anti-selfadjoint. Because of that, the contribution of the part of $D$ which is homogeneous in $u$ of order $\kappa$ to (4.1.10) will be expressed through a commutator, and will provide a remainder of type $\|u\|_{H^{s}}^{2 \kappa+2}$. As explained above, the terms coming from the part $D_{0}$ of $D$ independent of $u$ will cancel out (4.1.8) if the symbol $a$ is conveniently defined in terms of $b$. Finally, since for small functions $u$, (4.1.7) will be equivalent to $\|u(t, \cdot)\|_{H^{s}}^{2}$ we shall get

$$
\frac{d}{d t}\|u(t, \cdot)\|_{H^{s}}^{2} \leq C\|u(t, \cdot)\|_{H^{s}}^{2 \kappa+2}
$$

as long as $\|u(t, \cdot)\|_{H^{s}}$ stays small enough, which is what we need to get a solution defined on an interval of length $c \epsilon^{-2 \kappa}$. 
Let us mention that the computations we outlined above will have to be done using paradifferential operators instead of pseudo-differential ones. This is the justification for our study of the former in section 2 . The diagonalization of the principal symbol of the equation, i.e. the construction of $\tilde{u}$ in terms of $u$, will be described in subsection 4.2. The last subsection 4.3 will be devoted to the construction of the correcting terms (4.1.9) and to the proof of the theorem.

4.2. Diagonalization of principal part. We shall denote $\Lambda_{m}=\sqrt{-\Delta+V+m^{2}}$. This is a scalar invertible pseudo-differential operator of order 1 on $\mathbb{S}^{1}$. If $v \in$ $H^{s+1}\left(\mathbb{S}^{1}, \mathbb{R}\right)$ for a large enough $s$, we set

$$
u=\left[\begin{array}{c}
\Lambda_{m} v \\
\partial_{t} v
\end{array}\right], v=\Lambda_{m}^{-1} u_{1}, \partial_{t} v=u_{2}
$$

We define

$$
a(u)=c\left(\Lambda_{m}^{-1} u_{1}, u_{2}, \partial_{x} \Lambda_{m}^{-1} u_{1}\right),
$$

where $c$ is the function defined in (1.1.1), (1.1.2). In particular, $a(u)$ may be written as a sum of multilinear expressions in $T u_{1}, u_{2}$ for pseudo-differential operators of order $0, T$. Consequently $a(u)$ will be, according to Definitions 3.3.1 and 3.2.1, a symbol of $\widetilde{S}_{\mathrm{sc}}^{0}$ (independent of $n$ ). Its valuation will be equal to $\kappa$, which, according to assumption (1.1.3), may be assumed to be odd. Moreover it will satisfy condition $C(\kappa, r)$ of Definition 3.3 .2 i.e.,

$$
a=\sum_{k=\kappa}^{\kappa_{1}} a_{k}(u) \text { where } a_{k} \in S_{k}^{0}, a_{2 k} \equiv 0 \text { for } \kappa \leq 2 k<r-1 .
$$

The first equation of (1.1.4) may be written

$$
\partial_{t} u=\left[\begin{array}{cc}
0 & \Lambda_{m} \\
-(1+a(u))^{2} \Lambda_{m} & 0
\end{array}\right] u .
$$

We shall denote by $G$ the vector space $\mathbb{R}^{2}$, and consider the operator $-\frac{d^{2}}{d x^{2}}+$ $V(x)$ acting on $L^{2}\left(\mathbb{S}^{1}, G\right)$. As in section 2.1 , we denote by $\left(\omega_{n}^{-}\right)^{2} \leq\left(\omega_{n}^{+}\right)^{2}$ the couple of eigenvalues with asymptotics (1.2.1), and by $\Pi_{n}$ the spectral projector on the subspace of $L^{2}\left(\mathbb{S}^{1}, G\right)$ generated by the eigenfunctions associated to these two eigenvalues for $n \geq \tau+1$ large enough. We denote by $E_{n}$ the range of $\Pi_{n}$. Then $E_{n}$ is four dimensional for $n \geq \tau+1$. We define $E_{\tau}$ to be the orthogonal complement in $L^{2}\left(\mathbb{S}^{1}, G\right)$ of the Hilbert sum $\bigoplus_{n \geq \tau+1} E_{n}$. Then $E_{\tau}$ is even dimensional and we have the Hilbert decomposition

$$
L^{2}\left(\mathbb{S}^{1}, G\right)=\bigoplus_{n=\tau}^{+\infty} E_{n}
$$

At times we shall denote by $E_{n}^{\prime}, n \geq \tau+1$ the subspace of $L^{2}\left(\mathbb{S}^{1}, \mathbb{R}\right)$ generated by the two eigenfunctions associated to the eigenvalues $\left(\omega_{n}^{-}\right)^{2}$ and $\left(\omega_{n}^{+}\right)^{2}$ of the operator $-\frac{d^{2}}{d x^{2}}+V(x)$ acting on $L^{2}\left(\mathbb{S}^{1}, \mathbb{R}\right)$. We define $E_{\tau}^{\prime}$ in a similar way as $E_{\tau}$. We have for $n \geq \tau, E_{n} \simeq E_{n}^{\prime} \times E_{n}^{\prime}$. We denote by $\mathcal{E}$ the algebraic direct sum of $E_{n}$ for $n \geq \tau$. We introduce the following matrices:

$$
P(u, n)=\left[\begin{array}{cc}
\mathrm{I}_{K^{\prime}(n)} & \mathrm{I}_{K^{\prime}(n)} \\
i(1+a(u)) \mathrm{I}_{K^{\prime}(n)} & -i(1+a(u)) \mathrm{I}_{K^{\prime}(n)}
\end{array}\right]
$$


and

$$
Q(u, n)=\frac{i}{2}\left[\begin{array}{cc}
-i(1+a(u)) \mathrm{I}_{K^{\prime}(n)} & -\mathrm{I}_{K^{\prime}(n)} \\
-i(1+a(u)) \mathrm{I}_{K^{\prime}(n)} & \mathrm{I}_{K^{\prime}(n)}
\end{array}\right]
$$

so that

$$
P(u, n) Q(u, n)=Q(u, n) P(u, n)=(1+a(u)) \mathrm{I}_{2 K^{\prime}(n)},
$$

where $K^{\prime}(n)=\operatorname{dim} E_{n}^{\prime}=2$ when $n>\tau$. (We prefer to use $Q(u, n)$ instead of $P(u, n)^{-1}$ to always work with matrices whose coefficients are polynomial in $u$.) Then, according to Definitions 3.3.1 and 3.2.1, $P$ and $Q$ are elements of $\widetilde{S}^{0}$. Actually these matrices define, according to Definition 3.2.6 and (3.2.33) elements of $\widetilde{S}_{\mathrm{sc}}^{0}$, since each block of $P(u, n), Q(u, n)$ is a scalar matrix (the contribution of order $-\infty$ of Definition 3.2.6 is zero in this case). Moreover

$$
v(P)=v(Q)=0, v^{\prime}(P)=v^{\prime}(Q)=\kappa
$$

and $P(u, n)$ and $Q(u, n)$ satisfy condition $C(\kappa, r)$.

Recall that we have constructed in Theorem 1.2 .1 a nice basis of $L^{2}\left(\mathbb{S}^{1}, \mathbb{R}\right)$, which was adapted to the decomposition given by the $E_{n}^{\prime}$ (which were then denoted by $\left.E_{n}\right)$. We construct from this nice basis a natural basis of $E_{n}=E_{n}^{\prime} \times E_{n}^{\prime}$, which makes a nice basis of $L^{2}\left(\mathbb{S}^{1}, G\right)$, as at the beginning of subsection 3.2. We denote by $\lambda_{m}(n)$ the matrix of $\left.\Lambda_{m}\right|_{E_{n}^{\prime}}$ in the above nice basis. For $n \geq \tau+1, \lambda_{m}(n)$ is a $2 \times 2$ matrix. We denote by $\omega(\lambda)$ a symbol of order 1 on $\mathbb{R}_{+}$with asymptotics given by (1.2.1) and we define

$$
\omega_{m}(n)=\sqrt{m^{2}+\omega(n)^{2}}
$$

so that the difference between the eigenvalues of $\left.\sqrt{-\Delta+V+m^{2}}\right|_{E_{n}^{\prime}}$ and $\omega_{m}(n)$ is $O\left(n^{-\infty}\right)$ when $n \rightarrow+\infty$. The matrix $\lambda_{m}(n)$ may be written as

$$
\lambda_{m}(n)=\omega_{m}(n) \mathrm{I}_{K^{\prime}(n)}+\hat{\lambda}_{m}(n),
$$

where $\hat{\lambda}_{m}(n)$ is a matrix whose norm decays like $n^{-\infty}$ when $n \rightarrow+\infty$. We introduce for $n \geq \tau$ the matrix

$$
M(u, n)=\left[\begin{array}{cc}
0 & \lambda_{m}(n) \\
-(1+a(u))^{2} \lambda_{m}(n) & 0
\end{array}\right] .
$$

This is a $K(n) \times K(n)$ matrix (where $K(n)=\operatorname{dim} E_{n}=2 K^{\prime}(n)$ ) and since $a(u) \in$ $\widetilde{S}^{0}$, we get that $M(u, \cdot) \in \widetilde{S}^{1}$. Actually, decomposition (4.2.11) shows that $M(u, \cdot) \in$ $\widetilde{S}_{\mathrm{Sc}}^{1}$ since we may write it as the sum of $\left[\begin{array}{cc}0 & \omega_{m}(n) \operatorname{Id}_{K^{\prime}(n)} \\ -(1+a(u))^{2} \omega_{m}(n) \operatorname{Id}_{K^{\prime}(n)} & 0\end{array}\right]$, which is scalar by blocks, and of a contribution of order $-\infty$. Moreover the coefficients of $M(u, n)$ satisfy condition $C(\kappa, r)$.

According to Definition 3.2.2, $\widetilde{\mathrm{Op}}(M(u, \cdot)) u$ is nothing but the right hand side of (4.2.4). We may thus write this equation as

$$
\partial_{t} u=\widetilde{\mathrm{Op}}(M(u, \cdot)) u \text {. }
$$

Let us introduce the energy of solutions of (4.2.13) that we shall consider. We denote by $\widetilde{\Lambda}_{m}$ the operator $\widetilde{\Lambda}_{m}=\widetilde{\mathrm{Op}}\left(\omega_{m}(n) \mathrm{I}_{K(n)}\right)$ acting on $L^{2}\left(\mathbb{S}^{1}, G\right)$, so that $\widetilde{\Lambda}_{m} \Pi_{n}=\omega_{m}(n) \Pi_{n}$. For $s$ large enough we set

$$
\Theta_{0}^{s}(u(t, \cdot))=2\left\langle\widetilde{\Lambda}_{m}^{s} \mathrm{Op}\left(Q_{\chi}(u ; \cdot)\right) u, \widetilde{\Lambda}_{m}^{s} \mathrm{Op}\left(Q_{\chi}(u ; \cdot)\right) u\right\rangle,
$$


where $\chi \in C_{0}^{\infty}(\mathbb{R}), \chi \equiv 1$ close to $0, \chi$ even, Supp $\chi$ small enough, and where $Q_{\chi} \in \widetilde{\Sigma}_{1}^{0, \nu}$ (for some $\nu \in \mathbb{R}_{+}$) is defined from $Q$ in Corollary 3.3.3 (see also (3.2.5) $)$. Because of (4.2.9),

$$
v\left(Q_{\chi}\right)=0, v^{\prime}\left(Q_{\chi}\right)=\kappa .
$$

The following lemma asserts that $\Theta_{0}^{s}(u)$ is indeed equivalent to $\|u\|_{H^{s}}^{2}$ for small $u$, and gives an alternative expression for $\Theta_{0}^{s}(u)$, which will be useful in the sequel.

Lemma 4.2.1. There is $s_{0}>0$ and for any $s \geq s_{0}$ there are constants $C>0, R_{0}>$ 0 such that for any $u \in H^{s}\left(\mathbb{S}^{1}, G\right)$ with $\|u\|_{H^{s_{0}}}<R_{0}$, one has

$$
C^{-1}\|u\|_{H^{s}}^{2} \leq \Theta_{0}^{s}(u) \leq C\|u\|_{H^{s}}^{2} .
$$

Moreover, we may find a selfadjoint scalar symbol $c(u, \cdot) \in \widetilde{\Sigma}_{1}^{2 s, \nu}$, for some $\nu>0$ independent of $s$, with $v(c) \geq \kappa$, and satisfying condition $C(\kappa, r)$, such that if $\tilde{u}=\operatorname{Op}\left(Q_{\chi}(u ; \cdot)\right) u$,

$$
\begin{array}{r}
\Theta_{0}^{s}(u)=\left\langle\widetilde{\Lambda}_{m}^{s} \mathrm{Op}\left(\left(1+a_{\chi}\right)(u ; \cdot)\right) \tilde{u}, \widetilde{\Lambda}_{m}^{s} \tilde{u}\right\rangle+\left\langle\widetilde{\Lambda}_{m}^{s} \tilde{u}, \widetilde{\Lambda}_{m}^{s} \mathrm{Op}\left(\left(1+a_{\chi}\right)(u ; \cdot)\right) \tilde{u}\right\rangle \\
+\langle\operatorname{Op}(c(u ; \cdot)) \tilde{u}, \tilde{u}\rangle .
\end{array}
$$

Proof. We prove first (4.2.17). Observe that the left hand side and the sum of the first two brackets on the right hand side of (4.2.17) are real, so if we find a symbol $c$ satisfying (4.2.17), the equality remains true replacing $c$ by $\frac{1}{2}\left(c+c^{\bullet}\right)$ where $c^{\bullet}$ is defined by (2.2.1). In other words, as soon as we have found a $c$, we can construct from it a selfadjoint one.

Compute the difference between $\frac{1}{2} \Theta_{0}^{s}(u)$ and the first bracket on the right hand side of (4.2.17). We get

$$
-\left\langle\widetilde{\Lambda}_{m}^{2 s} \mathrm{Op}\left(a_{\chi}(u ; \cdot)\right) \tilde{u}, \tilde{u}\right\rangle
$$

We may always write $\widetilde{\Lambda}_{m}^{2 s}$ as a paradifferential operator associated to the symbol of $\Sigma_{0,0}^{2 s, 0}$ given by

$$
\chi\left(\frac{n_{0}-n_{1}}{n_{0}+n_{1}}\right)\left(\frac{\omega_{m}\left(n_{0}\right)+\omega_{m}\left(n_{1}\right)}{2}\right)^{2 s} .
$$

Moreover $a_{\chi}$ defined from $a$ in Corollary 3.3 .3 belongs to $\widetilde{\Sigma}_{1}^{0, \nu}$ for some $\nu \in \mathbb{R}_{+}$. By Corollary 3.3.5 (i), we may thus write (4.2.18) as $\langle\mathrm{Op}(c(u ; \cdot)) \tilde{u}, \tilde{u}\rangle$ for some symbol $c \in \widetilde{\Sigma}_{1}^{2 s, \nu}$, for a new value of $\nu$ independent of $s$. This gives (4.2.17).

Before starting the proof of (4.2.16), let us express $u$ as a function of $\tilde{u}$ and conversely. Denote

$P_{0}(n)=P(0, n)=\left[\begin{array}{cc}\mathrm{I}_{K^{\prime}(n)} & \mathrm{I}_{K^{\prime}(n)} \\ i \mathrm{I}_{K^{\prime}(n)} & -i \mathrm{I}_{K^{\prime}(n)}\end{array}\right], Q_{0}(n)=Q(0, n)=\frac{i}{2}\left[\begin{array}{cc}-i \mathrm{I}_{K^{\prime}(n)} & -\mathrm{I}_{K^{\prime}(n)} \\ -i \mathrm{I}_{K^{\prime}(n)} & \mathrm{I}_{K^{\prime}(n)}\end{array}\right]$.

If we denote $\sigma_{0}(u ; n)=Q_{\chi}(u ; n)-Q_{0, \chi}(n)$, we get a symbol in $\widetilde{\Sigma}_{1}^{0, \nu}$ for some $\nu$, with $v\left(\sigma_{0}\right) \geq \kappa$, satisfying condition $C(\kappa, r)$, such that by definition of $\tilde{u}$,

$$
\tilde{u}=Q_{0} u+\operatorname{Op}\left(\sigma_{0}(u ; \cdot)\right) u,
$$

where for short we write $Q_{0}$ for $\widetilde{\mathrm{Op}}\left(Q_{0}(\cdot)\right)=\mathrm{Op}\left(Q_{0, \chi}(\cdot)\right)$. Multiplying by $P_{0}=Q_{0}^{-1}$ we get, using the same type of notation convention,

$$
u=P_{0} \tilde{u}+\operatorname{Op}\left(\tilde{\sigma}_{0}(u ; \cdot)\right) u
$$


for another symbol $\tilde{\sigma}_{0}$ with $\tilde{\sigma}_{0} \in \widetilde{\Sigma}_{1}^{0, \nu}, v\left(\tilde{\sigma_{0}}\right) \geq \kappa, \tilde{\sigma}_{0}$ satisfying $C(\kappa, r)$. Using Proposition 2.1.3, we obtain that there are $C>0, s_{0}>0$ and for any $s \geq s_{0}$, there is $R_{0}>0$ small enough such that for any $u \in H^{s}$ with $\|u\|_{H^{s_{0}}}<R_{0}$,

$$
C^{-1}\|\tilde{u}\|_{H^{s}} \leq\|u\|_{H^{s}} \leq C\|\tilde{u}\|_{H^{s}},
$$

since the last terms in (4.2.20), (4.2.21) are $O\left(\|u\|_{H^{s_{0}}}^{\kappa}\|u\|_{H^{s}}\right), u \rightarrow 0$. If we apply Proposition 2.1.3 to the operators of order $2 s, \widetilde{\Lambda}_{m}^{2 s} \mathrm{Op}\left(a_{\chi}(u ; \cdot)\right)$ and $\mathrm{Op}(c(u ; \cdot))$, we see that there is a new value of $s_{0}$, independent of the order of these operators, such that for $s \geq s_{0}$ there is $C_{s}>0$ so that (4.2.18), as well as the last bracket in (4.2.17), is smaller than $C_{s}\|u\|_{H^{s_{0}}}^{\kappa}\|u\|_{H^{s}}^{2}$. This shows that

$$
\Theta_{0}^{s}(u)-2\left\langle\widetilde{\Lambda}_{m}^{s} \tilde{u}, \widetilde{\Lambda}_{m}^{s} \tilde{u}\right\rangle=O\left(\|u\|_{H^{s} s}^{\kappa}\|u\|_{H^{s}}^{2}\right), u \rightarrow 0 .
$$

Inequalities (4.2.16) follow from that and (4.2.22).

The interest of the preceding lemma is that it gives for $\Theta_{0}$ an expression in terms of $\tilde{u}$, and the equation written on $\tilde{u}$ will be essentially diagonal. Let us introduce some more notation. We set

$$
D(u, n)=Q(u, n) M(u, n) P(u, n)=i(1+a(u))^{2}\left[\begin{array}{cc}
\lambda_{m}(n) & 0 \\
0 & -\lambda_{m}(n)
\end{array}\right] .
$$

We also write

$$
D_{0}(n)=D(0, n), D^{\kappa}(u, n)=D(u, n)-D_{0}(n)
$$

so that $D^{\kappa}(u, n) \in \widetilde{S}_{\mathrm{sc}}^{1}$ with valuation greater than or equal to $\kappa$. Moreover $D(u, \cdot)$ satisfies condition $C(\kappa, r)$. We also set

$$
M_{0}(n)=M(0, n), M^{\kappa}(u, n)=M(u, n)-M_{0}(n)
$$

so that $M^{\kappa}(u, n)$ is an element of $\widetilde{S}_{\mathrm{sc}}^{1}$ of valuation greater than or equal to $\kappa$. In the same way, the expressions

$$
P^{\kappa}(u, n)=P(u, n)-P_{0}(n), Q^{\kappa}(u, n)=Q(u, n)-Q_{0}(n)
$$

are symbols of $\widetilde{S}_{\mathrm{sc}}^{0}$, with valuations greater than or equal to $\kappa$.

Lemma 4.2.2. There is some $\nu \in \mathbb{R}_{+}$and there are symbols $b_{0}(u ; \cdot)$ in $\widetilde{\Sigma}_{1}^{0, \nu}$, $b_{1}(u ; \cdot), \tilde{b}_{1}(u ; \cdot)$ in $\widetilde{\Sigma}_{1}^{1, \nu}$ with $v\left(b_{0}\right), v\left(\tilde{b}_{1}\right) \geq \kappa$, and there are operators $R, \tilde{R}$ in $\widetilde{\mathcal{R}}^{0, \nu}$, with $v(R), v(\tilde{R}) \geq \kappa+1$, satisfying condition $C(\kappa, r)$, such that one may write for every $u \in H^{s}\left(\mathbb{S}^{1}, G\right)$ that is a solution of (4.2.13),

$$
\begin{gathered}
\frac{\partial u}{\partial t}=P_{0} D_{0} \tilde{u}+\operatorname{Op}\left(\tilde{b}_{1}(u ; \cdot)\right) u+\tilde{R}(u), \\
\frac{\partial \tilde{u}}{\partial t}=\operatorname{Op}\left(b_{1}(u ; \cdot)\right) u+R(u), \\
\operatorname{Op}\left(\left(1+a_{\chi}\right)(u ; \cdot)\right) \frac{\partial \tilde{u}}{\partial t}=\operatorname{Op}\left(D_{\chi}(u ; \cdot)\right) \tilde{u}+\operatorname{Op}\left(b_{0}(u ; \cdot)\right) u+R(u),
\end{gathered}
$$

where we denoted by $D_{0}$ the operator $\widetilde{\mathrm{Op}}\left(D_{0}(n)\right)$.

Proof. Let us first show (4.2.27). We apply Corollary 3.3.3 to (4.2.13). We get

$$
\frac{\partial u}{\partial t}=\operatorname{Op}\left(M_{\chi}(u ; \cdot)\right) u+\operatorname{Op}\left(\tilde{b}_{0}(u ; \cdot)\right) u+\tilde{R}(u)
$$

where $\tilde{b}_{0} \in \widetilde{\Sigma}_{p}^{0, \nu}, \tilde{R} \in \widetilde{\mathcal{R}}^{0, \nu}$ for some $\nu \in \mathbb{R}_{+}, v\left(\tilde{b}_{0}\right) \geq \kappa, v(\tilde{R}) \geq \kappa+1, \tilde{b}_{0}$ and $\tilde{R}$ satisfying condition $C(\kappa, r)$. Using (4.2.25), we further decompose $\operatorname{Op}\left(M_{\chi}(u ; \cdot)\right)=$ 
$M_{0}+\operatorname{Op}\left(M_{\chi}^{\kappa}(u ; \cdot)\right)$, where $M_{0}$ denotes for short the operator with symbol $M_{0}(n)$. Since $M_{\chi}^{\kappa}(u, n) \in \widetilde{\Sigma}_{1}^{1, \nu}$ satisfies $v\left(M_{\chi}^{\kappa}\right) \geq \kappa$ and also satisfies condition $C(\kappa, r)$, we just have, to deduce (4.2.27) from (4.2.30), to express $M_{0} u$ in terms of $\tilde{u}$. This follows from (4.2.21) together with the expression $M_{0} P_{0}=P_{0} D_{0}$, which is a consequence of (4.2.23) and (4.2.8).

We shall now prove (4.2.28) and (4.2.29). We compute first

$$
\frac{\partial \tilde{u}}{\partial t}=\frac{\partial}{\partial t}\left[\mathrm{Op}\left(Q_{\chi}(u ; \cdot)\right) u\right]=\mathrm{Op}\left(Q_{\chi}(u ; \cdot)\right) \frac{\partial u}{\partial t}+\mathrm{Op}\left(Q_{\chi}^{\prime}(U ; \cdot)\right) u,
$$

where $U=\left(u, \partial_{t} u\right)$ and $Q^{\prime}(U, \cdot)$ is the symbol obtained by time derivation of $Q(u, \cdot)$. Let us show, using the equation, that $Q_{\chi}^{\prime}(U ; \cdot)$ is an element of $\widetilde{\Sigma}_{1}^{0, \nu}$ for some $\nu$, satisfying $v\left(Q_{\chi}^{\prime}(u ; \cdot)\right) \geq \kappa$ and satisfying condition $C(\kappa, r)$. By (4.2.7) we may write $Q_{\chi}^{\prime}(U ; \cdot)$ as a finite sum indexed by $p \geq \kappa$ of quantities of type

$$
a_{p, \chi}\left(\partial_{t} u, u, \ldots, u ; n_{0}, n_{p+1}\right)\left[\begin{array}{ll}
\frac{1}{2} \mathrm{I}_{K^{\prime}\left(n_{p+1}\right)} & 0 \\
\frac{1}{2} \mathrm{I}_{K^{\prime}\left(n_{p+1}\right)} & 0
\end{array}\right],
$$

where $a_{p}$ is the component homogeneous of degree $p$ in the expansion of $a$. If we plug in this expression (4.2.30), we see using Corollary 3.3.6 (i) and (ii) that we get a contribution of type $\operatorname{Op}\left(b_{0}(u ; \cdot)\right) u+R(u)$, like the last two terms on the right hand side of (4.2.29). In particular, such terms are of the form of the right hand side of (4.2.28). To finish the proof of (4.2.28), we just have to study the first term on the right hand side of (4.2.31). If we replace in that term $\partial_{t} u$ by (4.2.30) and use Corollaries 3.3.5 (i) and 3.3.6 (iii), we obtain that this contribution is again of the same form as the right hand side of (4.2.28). Let us prove (4.2.29), making $\operatorname{Op}\left(\left(1+a_{\chi}\right)(u ; \cdot)\right)$ act on (4.2.31). We have seen already that the last term on the right hand side of (4.2.31) has the structure of the last two terms on the right hand side of (4.2.29). This remains true if we make $\operatorname{Op}\left(\left(1+a_{\chi}\right)(u ; \cdot)\right)$ act on it, by Corollary 3.3.5 (i) and Corollary 3.3.6 (iii). So, we just have to study, using (4.2.30),

$$
\begin{aligned}
\operatorname{Op}\left(\left(1+a_{\chi}\right)(u ; \cdot)\right) \operatorname{Op}\left(Q_{\chi}(u ; \cdot)\right) \frac{\partial u}{\partial t}= & \operatorname{Op}\left(\left(1+a_{\chi}\right)(u ; \cdot)\right) \operatorname{Op}\left(Q_{\chi}(u ; \cdot)\right) \operatorname{Op}\left(M_{\chi}(u ; \cdot)\right) u \\
& +\mathrm{Op}\left(\left(1+a_{\chi}\right)(u ; \cdot)\right) \operatorname{Op}\left(Q_{\chi}(u ; \cdot)\right) \operatorname{Op}\left(\tilde{b}_{0}(u ; \cdot)\right) u \\
& +\mathrm{Op}\left(\left(1+a_{\chi}\right)(u ; \cdot)\right) \operatorname{Op}\left(Q_{\chi}(u ; \cdot)\right) \tilde{R}(u) .
\end{aligned}
$$

Again by Corollaries 3.3.5 (i) and 3.3.6 (iii), the last two terms give a contribution to the last two terms in (4.2.29). Since $a$ is a scalar symbol we may, by Corollary 3.3.5 (ii), commute in the first term on the right hand side of (4.2.32), $\mathrm{Op}\left(\left(1+a_{\chi}\right)(u ; \cdot)\right)$ and $\mathrm{Op}\left(Q_{\chi}(u ; \cdot)\right) \mathrm{Op}\left(M_{\chi}(u ; \cdot)\right)$, up to errors that may be incorporated inside the $\mathrm{Op}\left(b_{0}(u ; \cdot)\right) u$ term in (4.2.29). We are thus reduced to

$$
\operatorname{Op}\left(Q_{\chi}(u ; \cdot)\right) \operatorname{Op}\left(M_{\chi}(u ; \cdot)\right) \operatorname{Op}\left(\left(1+a_{\chi}\right)(u ; \cdot)\right) u .
$$

We apply Corollary 3.3 .4 to the symbols $P$ and $Q$ satisfying (4.2.8). Using also Corollary 3.3.5 (i) and Corollary 3.3.6 (iii), we obtain that 4.2.33) may be written as

$$
\left[\mathrm{Op}\left(Q_{\chi}(u ; \cdot)\right) \operatorname{Op}\left(M_{\chi}(u ; \cdot)\right) \operatorname{Op}\left(P_{\chi}(u ; \cdot)\right)\right] \operatorname{Op}\left(Q_{\chi}(u ; \cdot)\right) u,
$$

again up to contributions to the last two terms in (4.2.29). To conclude the proof, we just have to apply again Corollary 3.3 .4 to the bracket in the above formula, 
making use of the first equality (4.2.23) and of Corollaries 3.3.5 (i), 3.3.6 (iii) and 3.3 .7 (i).

We want to obtain a formula giving the time derivative of expressions generalizing the first term on the right hand side of (4.2.17). We introduce first some notation. We shall consider symbols $c \in \Sigma_{p, N_{0}}^{d, \nu}$ satisfying the following conditions:

$$
c(U ; \cdot)=c^{\prime}(U ; \cdot)+c^{\prime \prime}(U ; \cdot) \text { with } c^{\prime \prime} \in \Sigma_{p, N_{0}}^{d-1, \nu} \text { and selfadjoint, }
$$

$c^{\prime}(U ; \cdot)$ is selfadjoint and for any $n_{0}, n_{p+1} \geq \tau+1$,

$c^{\prime}\left(U ; n_{0}, n_{p+1}\right)=\left[\begin{array}{cc}c_{11}\left(U ; n_{0}, n_{p+1}\right) & 0 \\ 0 & c_{22}\left(U ; n_{0}, n_{p+1}\right)\end{array}\right]$ with $2 \times 2$ matrices $c_{11}, c_{22}$.

(Recall that our symbols of $\Sigma_{p, N_{0}}^{d, \nu}$ are $4 \times 4$ matrices when evaluated at $\left(n_{0}, n_{p+1}\right)$ with $n_{0}, n_{p+1} \geq \tau+1$.)

When $c \in \Sigma_{p, N_{0}}^{d, \nu}$ we shall associate to it the following function:

$$
c_{M_{0}}\left(u ; n_{0}, n_{p+1}\right)=\sum_{j=1}^{p} c\left(u, \ldots, M_{0} u, \ldots, u ; n_{0}, n_{p+1}\right),
$$

where as before $M_{0}$ denotes the operator with symbol $M_{0}(n)$, and where the term $M_{0} u$ is the $j$ th argument of the general term of the sum. We first prove a lemma.

Lemma 4.2.3. Let $\nu \in \mathbb{R}_{+}$. There is $\nu^{\prime} \in \mathbb{R}_{+}$such that for any $d \in \mathbb{R}, N_{0} \in$ $\mathbb{N}^{*}, p \in \mathbb{N}, c \in \Sigma_{p, N_{0}}^{d, \nu}$, one can find a symbol $e_{1} \in \widetilde{\Sigma}_{N_{0}}^{d, \nu^{\prime}}$ with $v\left(e_{1}\right) \geq \kappa+p$ and $R_{1} \in \widetilde{\mathcal{R}}^{d, \nu^{\prime}}$ with $v\left(R_{1}\right) \geq \kappa+p+1$, such that for any smooth enough solution $u$ of (4.2.13), and any smooth enough $v$,

$$
\mathrm{Op}\left(\frac{\partial}{\partial t} c(u, \ldots, u ; \cdot)\right) v=\mathrm{Op}\left(c_{M_{0}}(u ; \cdot)\right) v+\mathrm{Op}\left(e_{1}(u ; \cdot)\right) v+R_{1}(u, v) .
$$

Moreover, if $p$ is odd and $p \geq \kappa$, then $e_{1}$ satisfies condition $C(\kappa, r)$.

Proof. The left hand side of (4.2.37) is a sum of expressions

$$
\mathrm{Op}\left(c\left(u, \ldots, \frac{\partial u}{\partial t}, \ldots, u ; \cdot\right)\right) v .
$$

We use for $\frac{\partial u}{\partial t}$ expression (4.2.30) and decomposition (4.2.25). We get

$$
\frac{\partial u}{\partial t}=M_{0} u+\operatorname{Op}\left(M_{\chi}^{\kappa}(u ; \cdot)\right) u+\operatorname{Op}\left(\tilde{b}_{0}(u ; \cdot)\right) u+\tilde{R}(u) .
$$

When we plug this decomposition inside (4.2.38), we get from the $M_{0} u$ term, according to (4.2.36), a contribution to the first term on the right hand side of (4.2.37). The remaining terms in the above expression of $\frac{\partial u}{\partial t}$ contribute to the last two terms in (4.2.37), using (i) and (ii) of Corollary 3.3.6.

Let us now state the main proposition.

Proposition 4.2.4. Let $\nu \in \mathbb{R}_{+}, p \in \mathbb{N}, N_{0} \in \mathbb{N}^{*}$ be given. There is $\nu^{\prime} \in \mathbb{R}_{+}$and for any $d \in \mathbb{R}$, for any symbol $c \in \Sigma_{p, N_{0}}^{d, \nu}$ satisfying (4.2.34), (4.2.35), one can find - a selfadjoint symbol $e \in \widetilde{\Sigma}_{N_{0}}^{d, \nu^{\prime}}$ with $v(e) \geq p+\kappa$,

- an operator $R \in \widetilde{\mathcal{R}}^{d, \nu^{\prime}}$ satisfying $v(R) \geq p+\kappa+1$, 
such that for any smooth enough $u$ satisfying equation (4.2.13) one has, denoting $c(u ; \cdot)=c(u, \ldots, u ; \cdot)$,

$$
\begin{aligned}
\frac{1}{2} \frac{d}{d t}\left\langle\left[\operatorname{Op}(c(u ; \cdot)) \operatorname{Op}\left(\left(1+a_{\chi}\right)(u ; \cdot)\right)+\operatorname{Op}\left(\left(1+a_{\chi}\right)(u ; \cdot)\right)^{*} \operatorname{Op}(c(u ; \cdot))\right] \tilde{u}, \tilde{u}\right\rangle \\
=\left\langle\operatorname{Op}\left(c_{M_{0}}(u ; \cdot)\right) \tilde{u}, \tilde{u}\right\rangle+\left\langle\left[\operatorname{Op}(c(u ; \cdot)) D_{0}+D_{0}^{*} \operatorname{Op}(c(u ; \cdot))\right] \tilde{u}, \tilde{u}\right\rangle \\
+\langle\operatorname{Op}(e(u ; \cdot)) \tilde{u}, \tilde{u}\rangle+(\langle R(u), u\rangle+\langle u, R(u)\rangle) .
\end{aligned}
$$

Moreover, if $p$ is odd, $p \geq \kappa$, then $c_{M_{0}}, e, R$ satisfy condition $C(\kappa, r)$.

Proof. Remark that since $c$ is selfadjoint, so is $c_{M_{0}}$ defined by (4.2.36). So the left hand side and the first two terms on the right hand side of (4.2.39) are real-valued. Consequently, it is enough to prove (4.2.39) for some not necessarily selfadjoint symbol $e$, and replacing $(\langle R(u), u\rangle+\langle u, R(u)\rangle)$ by $\left(\left\langle R_{1}(u), u\right\rangle+\left\langle u, R_{2}(u)\right\rangle\right)$ for some $R_{1}, R_{2}$ satisfying the same conditions as $R$. Then taking real parts, we replace $e$ by $\frac{e+e^{\bullet}}{2}$ and $R_{j}$ by $\frac{R_{1}+R_{2}}{2}$ to get (4.2.39).

Let us show that we can write as the right hand side of (4.2.39) the time derivative

$$
\begin{aligned}
& \frac{d}{d t}\left\langle\operatorname{Op}(c(u ; \cdot)) \operatorname{Op}\left(\left(1+a_{\chi}\right)(u ; \cdot)\right) \tilde{u}, \tilde{u}\right\rangle \\
& \quad=\left\langle\operatorname{Op}\left(\frac{d}{d t} c(u ; \cdot)\right) \operatorname{Op}\left(\left(1+a_{\chi}\right)(u ; \cdot)\right) \tilde{u}, \tilde{u}\right\rangle+\left\langle\operatorname{Op}(c(u ; \cdot)) \operatorname{Op}\left(\frac{d}{d t} a_{\chi}(u ; \cdot)\right) \tilde{u}, \tilde{u}\right\rangle \\
& \quad+\left\langle\operatorname{Op}(c(u ; \cdot)) \operatorname{Op}\left(\left(1+a_{\chi}\right)(u ; \cdot)\right) \frac{d}{d t} \tilde{u}, \tilde{u}\right\rangle+\left\langle\operatorname{Op}(c(u ; \cdot)) \operatorname{Op}\left(\left(1+a_{\chi}\right)(u ; \cdot)\right) \tilde{u}, \frac{d}{d t} \tilde{u}\right\rangle .
\end{aligned}
$$

The idea of the proof is the following: we shall express $\frac{\partial \tilde{u}}{\partial t}$ using (4.2.28) or (4.2.29). The linear contributions coming from these expressions will give the first two terms on the right hand side of (4.2.39). The contributions which are at least of order $\kappa$ in $u$ will contribute to the last two terms. The key point will be not to lose derivatives, i.e. to check that $e$ is of order $d$ and not $d+1$. This will follow from the fact that $\operatorname{Op}(e(u ; \cdot))$ will be expressed from commutators of operators with commuting symbols. Symbolic calculus will thus bring the needed gain of one derivative. Let us proceed with the implementation of such a strategy.

\section{Study of first term in RHS of (4.2.40).}

Let us consider

$$
\operatorname{Op}\left(\frac{d}{d t} c(u ; \cdot)\right) \operatorname{Op}\left(\left(1+a_{\chi}\right)(u ; \cdot)\right) \tilde{u}
$$

By Lemma 4.2.3, we may write this as

$$
\begin{array}{r}
\mathrm{Op}\left(c_{M_{0}}(u ; \cdot)\right) \operatorname{Op}\left(\left(1+a_{\chi}\right)(u ; \cdot)\right) \tilde{u}+\operatorname{Op}\left(e_{1}(u ; \cdot)\right) \operatorname{Op}\left(\left(1+a_{\chi}\right)(u ; \cdot)\right) \tilde{u} \\
+R_{1}\left(u, \operatorname{Op}\left(\left(1+a_{\chi}\right)(u ; \cdot)\right) \tilde{u}\right) .
\end{array}
$$

The first term gives on the one hand the first term on the right hand side of (4.2.39), and on the other hand a contribution $\operatorname{Op}\left(c_{M_{0}}(u ; \cdot)\right) \operatorname{Op}\left(a_{\chi}(u ; \cdot)\right) \tilde{u}$. Using Corollary 3.3 .5 (i), we see that this expression can be incorporated in the $\operatorname{Op}(e(u ; \cdot)) \tilde{u}$ term in (4.2.39). Remark that the index $\nu^{\prime}$ given by Corollary 3.3.5 is independent of the order $d$ of $c$. The second term in (4.2.41) gives similarly a contribution to the $e$-term in (4.2.39). In the last term, we express $\tilde{u}$ from $u$ using (4.2.20). From Corollaries 3.3 .5 (i) and 3.3 .7 (i), we see that we obtain a contribution $\langle R(u), \tilde{u}\rangle$ 
for some $R$ satisfying the requirements of the statement of Proposition 4.2.4. If we express $\tilde{u}$ from $u$ by (4.2.20) and use (iii) of Corollary 3.3.6, we see that we obtain a contribution to the fourth term on the right hand side of (4.2.39).

Study of second term in RHS of (4.2.40).

If we apply Lemma 4.2 .3 to the symbol $a_{\chi}$ of order 0 , we see that

$$
\operatorname{Op}\left(\frac{d}{d t} a_{\chi}(u ; \cdot)\right) \tilde{u}=\operatorname{Op}\left(a_{\chi, M_{0}}(u ; \cdot)\right) \tilde{u}+\operatorname{Op}\left(e_{1}(u ; \cdot)\right) \tilde{u}+R_{1}(u, \tilde{u}),
$$

where $a_{\chi, M_{0}} \in \widetilde{\Sigma}_{1}^{0, \nu}, e_{1} \in \widetilde{\Sigma}_{1}^{0, \nu^{\prime}}, R_{1} \in \widetilde{\mathcal{R}}^{0, \nu^{\prime}}$ for some $\nu^{\prime} \in \mathbb{R}_{+}$, with moreover $v\left(a_{\chi, M_{0}}\right) \geq \kappa, v\left(e_{1}\right) \geq 2 \kappa, v\left(R_{1}\right) \geq 2 \kappa+1$. If we make $\operatorname{Op}(c(u ; \cdot))$ act on the left and use as before Corollaries 3.3.5 (i), 3.3.6 (iii), (4.2.20) and Corollary 3.3.7 (i), we obtain a contribution to the third and fourth terms in (4.2.39).

Study of third and fourth terms in RHS of (4.2.40).

We write the sum of the last two terms in (4.2.40) as

$$
2 \operatorname{Re}\left\langle\operatorname{Op}(c(u ; \cdot)) \operatorname{Op}\left(\left(1+a_{\chi}\right)(u ; \cdot)\right) \frac{d \tilde{u}}{d t}, \tilde{u}\right\rangle+\left\langle\left[\operatorname{Op}(c(u ; \cdot)), \operatorname{Op}\left(a_{\chi}(u ; \cdot)\right)\right] \tilde{u}, \frac{d \tilde{u}}{d t}\right\rangle
$$

using that $c$ and $a_{\chi}$ are selfadjoint symbols. We may apply Corollary 3.3.5 (ii) to the bracket in (4.2.42), since $a_{\chi}$ is scalar and so commutes with $c$. There is $\nu^{\prime}$, independent of $d$, and a symbol $b \in \widetilde{\Sigma}_{N_{0}}^{d-1, \nu^{\prime}}$ with $v(b) \geq \kappa+p$ such that the last term in (4.2.42) equals

$$
\left\langle\mathrm{Op}(b(u ; \cdot)) \tilde{u}, \frac{d \tilde{u}}{d t}\right\rangle
$$

Using (4.2.28), we reduce ourselves to the study of

$$
\left\langle\tilde{u}, \mathrm{Op}(b(u ; \cdot))^{*}\left(\mathrm{Op}\left(b_{1}(u ; \cdot)\right) u+R(u)\right)\right\rangle .
$$

Using, as in the study of the preceding cases, (4.2.20), and Corollaries 3.3.5 (i) and 3.3.6 (iii), we may write this expression as a contribution to the third and fourth terms on the right hand side of (4.2.39), using that the sum of the orders of the involved symbols is at most $d$.

Let us study now the first term in (4.2.42). We write using (4.2.29),

$$
\begin{array}{r}
\mathrm{Op}(c(u ; \cdot)) \operatorname{Op}\left(\left(1+a_{\chi}\right)(u ; \cdot)\right) \frac{d \tilde{u}}{d t}=\operatorname{Op}(c(u ; \cdot)) \operatorname{Op}\left(D_{\chi}(u ; \cdot)\right) \tilde{u} \\
+\mathrm{Op}(c(u ; \cdot)) \operatorname{Op}\left(b_{0}(u ; \cdot)\right) u \\
+\mathrm{Op}(c(u ; \cdot)) R(u) .
\end{array}
$$

The contribution of the last two terms to the first duality bracket in (4.2.42) is in the form of the conjugate of (4.2.43), since the sum of the orders of the symbols is at most $d$, and $R(\cdot) \in \widetilde{\mathcal{R}}^{0, \nu}$, and has already been treated. To study the first term on the right hand side of (4.2.44), where $D_{\chi}$ is a symbol of order 1, recall decomposition (4.2.24), which allows us to write

$$
D_{\chi}(u ; \cdot)=D_{0, \chi}(\cdot)+D_{\chi}^{\kappa}(u ; \cdot)
$$

We study first the contribution of the last term, i.e.

$$
\begin{aligned}
& 2 \operatorname{Re}\left\langle\operatorname{Op}(c(u ; \cdot)) \operatorname{Op}\left(D_{\chi}^{\kappa}(u ; \cdot)\right) \tilde{u}, \tilde{u}\right\rangle \\
& \quad=\left\langle\left[\operatorname{Op}(c(u ; \cdot)) \operatorname{Op}\left(D_{\chi}^{\kappa}(u ; \cdot)\right)+\operatorname{Op}\left(D_{\chi}^{\kappa}(u ; \cdot)\right)^{*} \operatorname{Op}(c(u ; \cdot))^{*}\right] \tilde{u}, \tilde{u}\right\rangle .
\end{aligned}
$$


Recall decomposition (4.2.34) of $c$. Since $c^{\prime \prime} \in \Sigma_{p, N_{0}}^{d-1, \nu}$, we may write by Corollary 3.3 .5 (i) $\mathrm{Op}\left(c^{\prime \prime}(u ; \cdot)\right) \operatorname{Op}\left(D_{\chi}^{\kappa}(u ; \cdot)\right)=\operatorname{Op}(g(u ; \cdot))$ for a new symbol $g \in \widetilde{\Sigma}_{N_{0}}^{d, \nu^{\prime}}$ with $\nu^{\prime}$ independent of $d$ and $v(g) \geq p+\kappa$. This term will give in (4.2.46) a contribution which can be treated as (4.2.43). The $c^{\prime}$ contribution to (4.2.46) may be written, since $c^{\prime}$ is selfadjoint,

$$
\left\langle\left[\mathrm{Op}\left(c^{\prime}(u ; \cdot)\right) \operatorname{Op}\left(D_{\chi}^{\kappa}(u ; \cdot)\right)+\operatorname{Op}\left(D_{\chi}^{\kappa}(u ; \cdot)\right)^{*} \mathrm{Op}\left(c^{\prime}(u ; \cdot)\right)\right] \tilde{u}, \tilde{u}\right\rangle .
$$

By (4.2.23), (4.2.24) and (4.2.11), we may decompose

$$
D^{\kappa}(u, n)=D^{\kappa \prime}(u, n)+\hat{D}^{\kappa}(u, n)
$$

with

$$
\begin{aligned}
& D^{\kappa \prime}(u, n)=i\left(2 a(u)+a(u)^{2}\right) \omega_{m}(n)\left[\begin{array}{cc}
\mathrm{I}_{K^{\prime}(n)} & 0 \\
0 & -\mathrm{I}_{K^{\prime}(n)}
\end{array}\right], \\
& \hat{D}^{\kappa}(u, n) \text { of order }-\infty .
\end{aligned}
$$

The contribution of $\hat{D}^{\kappa}(u, n)$ to (4.2.47) may be treated as expression (4.2.43). Since we may write $D^{\kappa \prime}(u, n)^{*}=-D^{\kappa \prime}(u, n)$, (ii) of Proposition 3.2.7 shows that $\operatorname{Op}\left(D_{\chi}^{\kappa \prime}(u ; \cdot)\right)^{*}=-\mathrm{Op}\left(D_{\chi}^{\kappa \prime}(u ; \cdot)\right)$ modulo an operator of order zero, whose contribution may be treated as (4.2.43). Consequently, we are left with

$$
\left\langle\left[\mathrm{Op}\left(c^{\prime}(u ; \cdot)\right), \operatorname{Op}\left(D^{\kappa \prime}(u ; \cdot)\right)\right] \tilde{u}, \tilde{u}\right\rangle .
$$

Observe now that by assumption (4.2.35) and the expression of $D^{\kappa \prime}$, we have $c^{\prime}(u, \cdot) \circ$ $D^{\kappa \prime}(u, \cdot)=D^{\kappa \prime}(u, \cdot) \circ c^{\prime}(u, \cdot)$ (for large enough phase arguments of the symbols). We may therefore apply Corollary 3.3.5 (ii) to write the commutator as an operator associated to a symbol in $\widetilde{\Sigma}_{N_{0}}^{d, \nu^{\prime}}$, of valuation greater than or equal to $\kappa+p$, for some $\nu^{\prime}$ independent of $d$. Reasoning as for (4.2.43), we get again a contribution to the last two terms in (4.2.39).

To finish the proof, we just have to notice that the contribution to the first term in (4.2.42) obtained by plugging the first term in the right hand side of (4.2.45) inside the first term in the right hand side of (4.2.44) gives the second term in the right hand side of (4.2.39). This concludes the proof of the proposition.

Proposition 4.2.5. Let $\nu \in \mathbb{R}_{+}$. There is $\nu^{\prime} \in \mathbb{R}_{+}$and for any $p \in \mathbb{N}^{*}, d \in \mathbb{R}, \widetilde{M} \in$ $\mathcal{R}_{p}^{d, \nu}$, there are operators $R_{1} \in \widetilde{\mathcal{R}}^{d+1, \nu^{\prime}}, R_{2} \in \widetilde{\mathcal{R}}^{0, \nu^{\prime}}$ with $v\left(R_{1}\right) \geq \kappa+p, v\left(R_{2}\right) \geq$ $\kappa+1$, such that for any smooth enough u solving equation (4.2.13),

$$
\begin{aligned}
\frac{d}{d t}\langle\widetilde{M}(u, \ldots, u), u\rangle=\sum_{j=1}^{p} & \left\langle\widetilde{M}\left(u, \ldots, M_{0} u, \ldots, u\right), u\right\rangle+\left\langle M_{0}^{*} \widetilde{M}(u, \ldots, u), u\right\rangle \\
& +\left\langle R_{1}(u), u\right\rangle+\left\langle\widetilde{M}(u, \ldots, u), R_{2}(u)\right\rangle .
\end{aligned}
$$

Proof. We compute first $\widetilde{M}\left(\frac{d u}{d t}, u, \ldots, u\right)$ using formulas (4.2.30) and decomposing

$$
\mathrm{Op}\left(M_{\chi}(u ; \cdot)(u ; \cdot)\right)=M_{0} u+\operatorname{Op}\left(M_{\chi}^{\kappa}(u ; \cdot)(u ; \cdot)\right) .
$$

Using Corollary 3.3.7 we get a contribution to the first and third terms on the right hand side of (4.2.49). In the same way, we get from $\left\langle\widetilde{M}(u, \ldots, u), \frac{d u}{d t}\right\rangle$, using Corollary 3.3.6 (iii), contributions to the last two terms in (4.2.49). 
4.3. Refined energy inequalities and proof of the main theorem. The objective of this subsection is to prove Proposition 4.3 .2 below, which will imply Theorem 1.1.1. Recall that we defined in (4.2.14) for a solution $u$ of equation (4.2.4) the quantity $\Theta_{0}^{s}(u(t, \cdot))$, which, as long as $\|u(t, \cdot)\|_{H^{s}}$ remains small enough, is equivalent to $\|u(t, \cdot)\|_{H^{s}}^{2}$. We shall see that $\frac{d}{d t} \Theta_{0}^{s}(u(t, \cdot))$ may be written essentially as $\langle\operatorname{Op}(a(u ; \cdot)) \tilde{u}, \tilde{u}\rangle$ for a symbol $a$ of order $2 s$ and valuation $\kappa$. We shall next find a correction $\Theta_{1}^{s}(u(t, \cdot))$ so that $\frac{d}{d t}\left(\Theta_{0}^{s}(u(t, \cdot))-\Theta_{1}^{s}(u(t, \cdot))\right)$ may be written as $\langle\mathrm{Op}(b(u ; \cdot)) \tilde{u}, \tilde{u}\rangle$ with $b$ of order $2 s$ and valuation $r-1>\kappa$. This gain on the valuation will give us the long-time existence result we look for. The correction $\Theta_{1}^{s}$ will be constructed by solving an equation on symbols involving the right hand side of (4.2.39). This is the main technical part of this subsection.

Let us first recall some notation and a result of 10 that will play a crucial role. Recall from subsection 1.2 that the large eigenvalues of $P=\sqrt{-\Delta+V}$ come by pairs $\omega_{-}(n) \leq \omega_{+}(n)$ having the same asymptotics (1.2.1). We denote as before by $\omega(\cdot)$ a symbol on $\mathbb{R}_{+}$with asymptotics (1.2.1) at infinity. We fix a large enough integer $\tau$ so that the spectrum $\mathcal{H}$ of $P$ may be written as

$$
\mathcal{H}=\left(\mathcal{H} \cap I_{\tau}\right) \cup \bigcup_{n=\tau+1}^{+\infty}\left(\mathcal{H} \cap I_{n}\right),
$$

where for $n \geq \tau+1, I_{n}$ are disjoint intervals of length $O\left(n^{-\infty}\right)$ centered at $\omega(n)$ and containing $\omega_{-}(n)$ and $\omega_{+}(n)$, and where $I_{\tau}$ contains the small eigenvalues. We set $\widetilde{\mathcal{H}}=\mathcal{H} \cup\{\omega(n) ; n \in \mathbb{N}\}$ and write for $\widetilde{\mathcal{H}}$ a decomposition of the form (4.3.1). The decomposition of $L^{2}\left(\mathbb{S}^{1}, \mathbb{R}^{2}\right)$ associated to (4.3.1) is given by (4.2.5). Let us recall a special case of Proposition 2.2.1] of [10]. We use notation (2.1.5).

Proposition 4.3.1. For any $\xi \in \mathcal{H}$ (or $\widetilde{\mathcal{H}}$ ), denote by $n(\xi)$ the unique $n \in \mathbb{N}_{\tau}$ such that $\xi \in I_{n(\xi)}$. Let $p$ be an odd positive integer. There is a zero measure subset $\mathcal{N}$ of $] 0,+\infty[$ such that for any $m \in] 0,+\infty\left[-\mathcal{N}\right.$, there are $c>0, N_{0} \in \mathbb{N}$, so that for any $\xi_{0}, \ldots, \xi_{p+1} \in \mathcal{H}$ (or $\widetilde{\mathcal{H}}$ ), any $0 \leq q \leq p+1$,

$$
\left|\sum_{j=0}^{q} \sqrt{m^{2}+\xi_{j}^{2}}-\sum_{j=q+1}^{p+1} \sqrt{m^{2}+\xi_{j}^{2}}\right| \geq c \mu\left(n\left(\xi_{0}\right), \ldots, n\left(\xi_{p+1}\right)\right)^{-N_{0}} .
$$

From now on, we fix a value of $m$ outside $\mathcal{N}$, and so an integer $N_{0}$. We shall state and prove a proposition relying on division by quantities of the form (4.3.2). We first need to introduce some notation. If $a$ is a paradifferential symbol, $a \in$ $\Sigma_{p, N_{0}}^{d, \nu}$, recall that for any $u_{1}, \ldots, u_{p} \in \mathcal{E}, n_{0}, n_{p+1} \in \mathbb{N}_{\tau}, a\left(u_{1}, \ldots, u_{p} ; n_{0}, n_{p+1}\right)$ is a $K\left(n_{0}\right) \times K\left(n_{p+1}\right)$ matrix, where for $n \in \mathbb{N}_{\tau}, K(n)$ is an even integer (and $K(n)=4$ if $n \geq \tau+1)$. We can write a block decomposition of $a$ involving $K\left(n_{0}\right) / 2$ lines and $K\left(n_{p+1}\right) / 2$ columns:

$$
\left[\begin{array}{ll}
* & * \\
* & *
\end{array}\right]
$$

We shall consider the following two assumptions.

$\left(H_{D}\right)$ In (4.3.3) each block outside the diagonal is zero.

$\left(H_{N D}\right)$ In (4.3.3) each block on the diagonal is zero. 
In accordance with notation (4.2.36), if $c^{1}$ is a symbol in $\Sigma_{p, N_{0}}^{d, \nu}$ we shall set

$$
c_{M_{0}}^{1}\left(u_{1}, \ldots, u_{p} ; n_{0}, n_{p+1}\right)=\sum_{j=1}^{p} c^{1}\left(u_{1}, \ldots, M_{0} u_{j}, \ldots, u_{p} ; n_{0}, n_{p+1}\right) .
$$

Proposition 4.3.2. Let $\nu \in \mathbb{R}_{+}$. There is $\nu^{\prime} \in \mathbb{R}_{+}$such that for any $d \in \mathbb{R}, p \in \mathbb{N}$ odd, $a \in \Sigma_{p, N_{0}}^{d, \nu}$ satisfying assumption $\left(H_{D}\right)$ (resp. assumption $\left(H_{N D}\right)$ ), we may find a symbol $c^{1} \in \Sigma_{p, N_{0}}^{d, \nu^{\prime}}$ satisfying $\left(H_{D}\right)$ (resp. a symbol $c^{1} \in \Sigma_{p, N_{0}}^{d-1, \nu^{\prime}}$ satisfying $\left.\left(H_{N D}\right)\right)$ such that

$$
\begin{aligned}
& c_{M_{0}}^{1}\left(u_{1}, \ldots, u_{p} ; n_{0}, n_{p+1}\right)+c^{1}\left(u_{1}, \ldots, u_{p} ; n_{0}, n_{p+1}\right) D_{0}\left(n_{p+1}\right) \\
& -D_{0}\left(n_{0}\right) c^{1}\left(u_{1}, \ldots, u_{p} ; n_{0}, n_{p+1}\right)=a\left(u_{1}, \ldots, u_{p} ; n_{0}, n_{p+1}\right) .
\end{aligned}
$$

Moreover, if a is selfadjoint, we may assume that $c^{1}$ is also selfadjoint.

Remark that the last statement follows from (4.3.5) and the fact that if $c^{1}$ satisfies (4.3.5), then $c^{1 \bullet}$ defined by (2.2.1) also satisfies (4.3.5) with the right hand side replaced by $a^{\bullet}$ (since $\left.D(n)^{*}=-D(n)\right)$.

The proof of (4.3.5) will use several lemmas. We remark first that we may extend $c^{1}$ and $a$, which are $\mathbb{R}$-multilinear maps in $\left(u_{1}, \ldots, u_{p}\right)$ as $\mathbb{C}$-multilinear maps. This allows us to make the change of function $u_{j} \rightarrow P_{0} u_{j}$ in (4.3.5), where $P_{0}$ is defined in (4.2.19) and satisfies by (4.2.23) $P_{0} D_{0}=M_{0} P_{0}$. This equation is thus equivalent to

$$
\begin{aligned}
\tilde{c}_{D_{0}}^{1}\left(u_{1}, \ldots, u_{p} ; n_{0}, n_{p+1}\right)+\tilde{c}^{1}\left(u_{1}, \ldots, u_{p} ; n_{0}, n_{p+1}\right) D_{0}\left(n_{p+1}\right) \\
\quad-D_{0}\left(n_{0}\right) \tilde{c}^{1}\left(u_{1}, \ldots, u_{p} ; n_{0}, n_{p+1}\right)=\tilde{a}\left(u_{1}, \ldots, u_{p} ; n_{0}, n_{p+1}\right)
\end{aligned}
$$

where we denoted

$$
\begin{aligned}
\tilde{a}\left(u_{1}, \ldots, u_{p} ; n_{0}, n_{p+1}\right) & =a\left(P_{0} u_{1}, \ldots, P_{0} u_{p} ; n_{0}, n_{p+1}\right), \\
\tilde{c}^{1}\left(u_{1}, \ldots, u_{p} ; n_{0}, n_{p+1}\right) & =c^{1}\left(P_{0} u_{1}, \ldots, P_{0} u_{p} ; n_{0}, n_{p+1}\right), \\
\tilde{c}_{D_{0}}^{1}\left(u_{1}, \ldots, u_{p} ; n_{0}, n_{p+1}\right) & =\sum_{j=1}^{p} c^{1}\left(P_{0} u_{1}, \ldots, P_{0} D_{0} u_{j}, \ldots, P_{0} u_{p} ; n_{0}, n_{p+1}\right) .
\end{aligned}
$$

We shall denote by $\Sigma_{p, N_{0}}^{d, \nu}(N)$ the space of functions $a$ of type (2.1.10), defined on $(\mathcal{E} \otimes \mathbb{C}) \times \cdots \times(\mathcal{E} \otimes \mathbb{C}) \times \mathbb{N}_{\tau} \times \mathbb{N}_{\tau}$ instead of $\mathcal{E} \times \cdots \times \mathcal{E} \times \mathbb{N}_{\tau} \times \mathbb{N}_{\tau}$, which are $\mathbb{C}$ - $p$ linear in $\left(u_{1}, \ldots, u_{p}\right)$ and satisfy condition $(\mathrm{i})_{\delta}$ of Definition 2.1.1 for some $\left.\delta \in\right] 0,1[$ small enough, and inequalities (2.1.12) only when $\alpha+\beta+\gamma \leq N$. We endow this space with the norm $|a|_{p, N_{0}, N}^{d, \nu}$ given by the best constant in inequality (2.1.12). Of course, $\Sigma_{p, N_{0}}^{d, \nu}$ is the restriction of $\bigcap_{N} \Sigma_{p, N_{0}}^{d, \nu}(N)$ to real arguments $\left(u_{1}, \ldots, u_{p}\right)$. If $c^{1} \in \Sigma_{p, N_{0}}^{d, \nu}(N)$ we denote by $L\left(c^{1}\right)$ the symbol defined by the left hand side of (4.3.6). Recall that by (4.2.23), (4.2.24), (4.2.11), the matrix $D_{0}(n)=D(0, n)$ may be decomposed as

$$
D_{0}(n)=D_{0}^{\prime}(n)+\hat{D}_{0}(n), D_{0}^{\prime}(n)=i \omega_{m}(n)\left[\begin{array}{cc}
\mathrm{I}_{K^{\prime}(n)} & 0 \\
0 & -\mathrm{I}_{K^{\prime}(n)}
\end{array}\right],
$$

where $\hat{D}_{0}(n)$ is a symbol of order $-\infty$. When $n=\tau$, we may take $D_{0}^{\prime}(\tau)=0$. We then decompose

$$
L\left(c^{1}\right)=L_{0}\left(c^{1}\right)+L_{1}\left(c^{1}\right)
$$


with, if $U^{\prime}=\left(u_{1}, \ldots, u_{p}\right)$,

$$
\begin{aligned}
L_{0}\left(c^{1}\right)\left(U^{\prime} ; n_{0}, n_{p+1}\right)= & \tilde{c}_{D_{0}}^{1}\left(U^{\prime} ; n_{0}, n_{p+1}\right)+\tilde{c}^{1}\left(U^{\prime} ; n_{0}, n_{p+1}\right) D_{0}^{\prime}\left(n_{p+1}\right) \\
& -D_{0}^{\prime}\left(n_{0}\right) \tilde{c}^{1}\left(U^{\prime} ; n_{0}, n_{p+1}\right)
\end{aligned}
$$

and

$$
L_{1}\left(c^{1}\right)\left(U^{\prime} ; n_{0}, n_{p+1}\right)=\tilde{c}^{1}\left(U^{\prime} ; n_{0}, n_{p+1}\right) \hat{D}_{0}\left(n_{p+1}\right)-\hat{D}_{0}\left(n_{0}\right) \tilde{c}^{1}\left(U^{\prime} ; n_{0}, n_{p+1}\right) .
$$

Remark that $L_{1}$ sends $\Sigma_{p, N_{0}}^{d, \nu}(N)$ into $\Sigma_{p, N_{0}}^{-\infty, 0}(N)$ since $\hat{D}_{0}$ is of order $-\infty$. On the other hand, if $c^{1}$ satisfies condition $\left(H_{D}\right), \tilde{c}^{1}\left(U^{\prime} ; n_{0}, n_{p+1}\right)$ commutes when $n_{0}, n_{p+1} \in \mathbb{N}_{\tau+1}$ with $D_{0}^{\prime}\left(n_{0}\right)$, whence

$$
L_{0}\left(c^{1}\right)\left(U^{\prime} ; n_{0}, n_{p+1}\right)=\tilde{c}_{D_{0}}^{1}\left(U^{\prime} ; n_{0}, n_{p+1}\right)+\tilde{c}^{1}\left(U^{\prime} ; n_{0}, n_{p+1}\right)\left(D_{0}^{\prime}\left(n_{p+1}\right)-D_{0}^{\prime}\left(n_{0}\right)\right) .
$$

Remark that because of definition (4.2.10) of $\omega_{m}, \omega_{m}\left(n_{p+1}\right)-\omega_{m}\left(n_{0}\right)$ satisfies when $\left|n_{p+1}-n_{0}\right| \leq \frac{1}{4}\left(n_{p+1}+n_{0}\right)$ inequalities (3.3.15). This shows that if

$$
\begin{aligned}
\Sigma_{p, N_{0}}^{d, \nu} & =\left\{a \in \bigcap_{N} \Sigma_{p, N_{0}}^{d, \nu}(N) ; a \text { satisfies }\left(H_{D}\right)\right\}, \\
\Sigma_{p, N_{0}}^{d, \nu}(N) & =\Sigma_{p, N_{0}}^{d, \nu} \cap \Sigma_{p, N_{0}}^{d, \nu}(N),
\end{aligned}
$$

then $L_{0}$ sends $\Sigma_{p, N_{0}}^{d, \nu}(N)$ into $\Sigma_{p, N_{0}}^{d, \nu+1}(N-1)$.

If $c^{1}$ satisfies assumption $\left(H_{N D}\right)$, then for $n_{0}, n_{p+1} \in \mathbb{N}_{\tau+1}$,

$$
\tilde{c}^{1}\left(U^{\prime} ; n_{0}, n_{p+1}\right) D_{0}^{\prime}\left(n_{0}\right)=-D_{0}^{\prime}\left(n_{0}\right) \tilde{c}^{1}\left(U^{\prime} ; n_{0}, n_{p+1}\right),
$$

whence

(4.3.14)

$$
L_{0}\left(c^{1}\right)\left(U^{\prime} ; n_{0}, n_{p+1}\right)=\tilde{c}_{D_{0}}^{1}\left(U^{\prime} ; n_{0}, n_{p+1}\right)+\tilde{c}^{1}\left(U^{\prime} ; n_{0}, n_{p+1}\right)\left(D_{0}^{\prime}\left(n_{p+1}\right)+D_{0}^{\prime}\left(n_{0}\right)\right) .
$$

If we define

$$
\begin{aligned}
\Sigma_{p, N_{0}}^{\prime \prime d, \nu} & =\left\{a \in \bigcap_{N} \Sigma_{p, N_{0}}^{d, \nu}(N) ; a \text { satisfies }\left(H_{N D}\right)\right\}, \\
\Sigma_{p, N_{0}}^{\prime \prime d, \nu}(N) & =\Sigma_{p, N_{0}}^{\prime \prime d, \nu} \cap \Sigma_{p, N_{0}}^{d, \nu}(N),
\end{aligned}
$$

we obtain that $L_{0}$ sends $\Sigma_{p, N_{0}}^{\prime \prime d, \nu}(N)$ into $\Sigma_{p, N_{0}}^{d+1, \nu}(N)$. Let us prove the following lemma:

Lemma 4.3.3. (i) For any $d \in \mathbb{R}, \nu \in \mathbb{R}_{+}, p \in \mathbb{N}, N \in \mathbb{N}$, the operator $L$ is injective on $\Sigma_{p, N_{0}}^{d, \nu}(N)$.

(ii) Let $F$ be a subspace of $\Sigma_{p, N_{0}}^{d, \nu}(N)$ such that there is a finite subset $K$ of $\mathbb{N}_{\tau} \times \mathbb{N}_{\tau}$ so that for any $a \in F, a\left(\cdot ; n_{0}, n_{p+1}\right) \equiv 0$ if $\left(n_{0}, n_{p+1}\right) \notin K$. Then $F$ is stable by $L$ and $L: F \rightarrow F$ is bijective.

Proof. (i) We denote by $\Pi_{n}$ the spectral projector on the space $E_{n} \otimes \mathbb{C}$, where $E_{n}$ is defined by the decomposition (4.2.5) of $L^{2}\left(\mathbb{S}^{1} ; \mathbb{R}^{2}\right)$. We shall use the notation $\Pi_{n}^{\prime}$ for the similar projector acting on $L^{2}\left(\mathbb{S}^{1} ; \mathbb{C}\right)$. For every $n$, we denote by $(\omega(n, \ell))_{\ell}$ the $K^{\prime}(n)$ eigenvalues of the restriction of $P=\sqrt{-\Delta+V}$ to the range of $\Pi_{n}^{\prime}$ acting on $L^{2}\left(\mathbb{S}^{1} ; \mathbb{C}\right)$. We choose an orthonormal basis of that range made of eigenfunctions of $P$ associated to these eigenvalues (this is not in general a nice basis). We write

$$
\Pi_{n}^{\prime}=\sum_{\ell} \Pi_{n}^{\prime \ell}
$$


the corresponding decomposition of $\Pi_{n}^{\prime}$. The sum in (4.3.16) is finite, and for $n \geq \tau+1$ made up of only two terms as the range of $\Pi_{n}^{\prime}$ is two dimensional. We set $\omega_{m}(n, \ell)=\sqrt{m^{2}+\omega(n, \ell)^{2}}$ and we have

$$
\Lambda_{m} \Pi_{n}^{\prime \ell}=\omega_{m}(n, \ell) \Pi_{n}^{\prime \ell}
$$

and $\left(\omega_{m}(n, \ell)\right)_{\ell}$ are the eigenvalues of the matrix $\lambda_{m}(n)$ defined in (4.2.11). We define

$$
J_{+}(n)=\left[\begin{array}{cc}
\mathrm{I}_{K^{\prime}(n)} & 0 \\
0 & 0
\end{array}\right], J_{-}(n)=\left[\begin{array}{cc}
0 & 0 \\
0 & \mathrm{I}_{K^{\prime}(n)}
\end{array}\right], J(n)=J_{+}(n)-J_{-}(n)
$$

and set

$$
\begin{gathered}
\Pi_{n}^{\ell,+}=\left[\begin{array}{cc}
\Pi_{n}^{\prime \ell} & 0 \\
0 & 0
\end{array}\right], \Pi_{n}^{\ell,-}=\left[\begin{array}{cc}
0 & 0 \\
0 & \Pi^{\prime \ell}
\end{array}\right], \\
\Pi_{n}^{+}=\left[\begin{array}{cc}
\Pi_{n}^{\prime} & 0 \\
0 & 0
\end{array}\right], \Pi_{n}^{-}=\left[\begin{array}{cc}
0 & 0 \\
0 & \Pi^{\prime}{ }_{n}
\end{array}\right],
\end{gathered}
$$

so that $\Pi_{n}=\Pi_{n}^{+}+\Pi_{n}^{-}$and, denoting by $D_{0}$ the operator with symbol $D_{0}(n)$ given by (4.2.23), (4.2.24),

$$
D_{0} \Pi_{n}^{\ell, \pm}= \pm i \omega_{m}(n, \ell) \Pi_{n}^{\ell, \pm} .
$$

By (4.3.8), we also have

$$
D_{0}^{\prime} \Pi_{n}^{ \pm}= \pm i \omega_{m}(n) \Pi_{n}^{ \pm} .
$$

Recall the map $\mathcal{F}_{n}: L^{2}\left(\mathbb{S}^{1} ; \mathbb{K}^{2}\right) \rightarrow \mathbb{K}^{K(n)}(\mathbb{K}=\mathbb{R}$ or $\mathbb{C}$ ) defined by (2.1.6) and set

$$
\widetilde{\Pi}_{n}^{\ell, \pm}=\mathcal{F}_{n} \circ \Pi_{n}^{\ell, \pm} \circ \mathcal{F}_{n}^{*}, \widetilde{\Pi}_{n}^{ \pm}=\mathcal{F}_{n} \circ \Pi_{n}^{ \pm} \circ \mathcal{F}_{n}^{*} .
$$

These are projectors on $\mathbb{K}^{K(n)}$ and we have

$$
\begin{aligned}
D_{0}(n) \widetilde{\Pi}_{n}^{\ell, \pm} & =\widetilde{\Pi}_{n}^{\ell, \pm} D_{0}(n)= \pm i \omega_{m}(n, \ell) \widetilde{\Pi}_{n}^{\ell, \pm}, \\
D_{0}^{\prime}(n) \widetilde{\Pi}_{n}^{ \pm} & =\widetilde{\Pi}_{n}^{ \pm} D_{0}^{\prime}(n)= \pm i \omega_{m}(n) \widetilde{\Pi}_{n}^{ \pm} .
\end{aligned}
$$

Let $c^{1} \in \Sigma_{p, N_{0}}^{d, \nu}(N)$ be such that $L\left(c^{1}\right)$ vanishes identically. Compose $L\left(c^{1}\right)$ (given by the left hand side of (4.3.6) $)$ on the left by $\widetilde{\Pi}_{n_{0}}^{\ell_{0}, \epsilon_{0}}$ and on the right by $\widetilde{\Pi}_{n_{p+1}}^{\ell_{p+1}, \epsilon_{p+1}}$, and evaluate it at

$$
\Pi_{n^{\prime}}^{\ell^{\prime} \epsilon^{\prime}} U^{\prime}=\left(\Pi_{n_{1}}^{\ell_{1}, \epsilon_{1}} u_{1}, \ldots, \Pi_{n_{p}}^{\ell_{p}, \epsilon_{p}} u_{p}\right),
$$

where $\epsilon_{j} \in\{+,-\}, j=0, \ldots, p+1$. We get

$$
\begin{aligned}
\sum_{j=1}^{p} \widetilde{\Pi}_{n_{0}}^{\ell_{0}, \epsilon_{0}} c^{1}\left(P_{0} \Pi_{n_{1}}^{\ell_{1}, \epsilon_{1}} u_{1}, \ldots, P_{0} D_{0} \Pi_{n_{j}}^{\ell_{j}, \epsilon_{j}} u_{j}, \ldots, P_{0} \Pi_{n_{p}}^{\ell_{p}, \epsilon_{p}} u_{p} ; n_{0}, n_{p+1}\right) \widetilde{\Pi}_{n_{p+1}}^{\ell_{p+1}, \epsilon_{p+1}} \\
+\widetilde{\Pi}_{n_{0}}^{\ell_{0}, \epsilon_{0}} c^{1}\left(P_{0} \Pi_{n_{1}}^{\ell_{1}, \epsilon_{1}} u_{1}, \ldots, P_{0} \Pi_{n_{p}}^{\ell_{p}, \epsilon_{p}} u_{p} ; n_{0}, n_{p+1}\right) D_{0}\left(n_{p+1}\right) \widetilde{\Pi}_{n_{p+1}}^{\ell_{p+1}, \epsilon_{p+1}} \\
-\widetilde{\Pi}_{n_{0}}^{\ell_{0}, \epsilon_{0}} D_{0}\left(n_{0}\right) c^{1}\left(P_{0} \Pi_{n_{1}}^{\ell_{1}, \epsilon_{1}} u_{1}, \ldots, P_{0} \Pi_{n_{p}}^{\ell_{p}, \epsilon_{p}} u_{p} ; n_{0}, n_{p+1}\right) \widetilde{\Pi}_{n_{p+1}}^{\ell_{p+1}, \epsilon_{p+1}} \equiv 0 .
\end{aligned}
$$

Using (4.3.20), (4.3.23) we may write this as

$$
i\left(\sum_{j=1}^{p+1} \epsilon_{j} \omega_{m}\left(n_{j}, \ell_{j}\right)-\epsilon_{0} \omega_{m}\left(n_{0}, \ell_{0}\right)\right) \widetilde{\Pi}_{n_{0}}^{\ell_{0}, \epsilon_{0}} \tilde{c}^{1}\left(\Pi_{n^{\prime}}^{\ell^{\prime}, \epsilon^{\prime}} U^{\prime} ; n_{0}, n_{p+1}\right) \widetilde{\Pi}_{n_{p+1}}^{\ell_{p+1}, \epsilon_{p+1}} \equiv 0 .
$$

Condition (4.3.2) shows that for $m$ outside $\mathcal{N}$, the scalar coefficient above never vanishes, which implies $\tilde{c}^{1} \equiv 0$, whence $c^{1} \equiv 0$. This proves (i) of the lemma. 
To prove (ii), we remark that if $a \in F$ is given, we may define $c^{1} \in F$ with $L\left(c^{1}\right)=a$ by

$$
\begin{aligned}
& \widetilde{\Pi}_{n_{0}}^{\ell_{0}, \epsilon_{0}} \tilde{c}^{1}\left(\Pi_{n^{\prime}}^{\ell^{\prime}, \epsilon^{\prime}} U^{\prime} ; n_{0}, n_{p+1}\right) \widetilde{\Pi}_{n_{p+1}}^{\ell_{p+1}, \epsilon_{p+1}} \\
& =-i\left(\sum_{j=1}^{p+1} \epsilon_{j} \omega_{m}\left(n_{j}, \ell_{l}\right)-\epsilon_{0} \omega_{m}\left(n_{0}, \ell_{0}\right)\right)^{-1} \widetilde{\Pi}_{n_{0}}^{\ell_{0}, \epsilon_{0}} \tilde{a}\left(\Pi_{n^{\prime}}^{\ell^{\prime}, \epsilon^{\prime}} U^{\prime} ; n_{0}, n_{p+1}\right) \widetilde{\Pi}_{n_{p+1}}^{\ell_{p+1}, \epsilon_{p+1}} .
\end{aligned}
$$

Since by definition of $F, n_{0}, n_{p+1}$ stay in a bounded set of indices, the estimates of definition of a symbol hold true trivially.

Proof of Proposition 4.3.2. Using the notation (4.3.13), 4.3.15), we shall construct operators

$$
\begin{aligned}
& L^{-1}: \Sigma_{p, N_{0}}^{d, \nu} \rightarrow \Sigma_{p, N_{0}}^{d, \nu+N_{0}}, \\
& L^{-1}: \Sigma_{p, N_{0}}^{\prime \prime d, \nu} \rightarrow \underset{p, N_{0}}{\Sigma^{\prime \prime d-1, \nu+N_{0}}}
\end{aligned}
$$

such that $L \circ L^{-1}=\mathrm{Id}$. This will give the wanted conclusion. It will be enough to construct for any $N$,

$$
\begin{aligned}
& L_{N}^{-1}: \Sigma_{p, N_{0}}^{d, \nu}(N) \rightarrow \Sigma_{p, N_{0}}^{d, \nu+N_{0}}(N+1), \\
& L_{N}^{-1}: \Sigma_{p, N_{0}}^{\prime \prime d, \nu}(N) \rightarrow \Sigma_{p, N_{0}}^{\prime \prime d-1, \nu+N_{0}}(N)
\end{aligned}
$$

such that $L \circ L_{N}^{-1}: \Sigma_{p, N_{0}}^{d, \nu}(N) \rightarrow \Sigma_{p, N_{0}}^{d, \nu+N_{0}+1}(N)$ and $L \circ L_{N}^{-1}: \Sigma_{p, N_{0}}^{\prime \prime d, \nu}(N) \rightarrow$ $\underset{p, N_{0}}{\Sigma^{\prime \prime} d, \nu+N_{0}}(N)$ coincide with the identity. Actually, since $L$ is injective by Lemma 4.3 .3 .

$$
\left.L_{N}^{-1}\right|_{\Sigma_{p, N_{0}}^{\prime d, \nu}(N+1)}=L_{N+1}^{-1},\left.L_{N}^{-1}\right|_{\Sigma^{\prime \prime}{ }_{p, N_{0}}^{d, \nu}(N+1)}=L_{N+1}^{-1},
$$

which allows us to define $L^{-1}$ satisfying (4.3.24).

If $A_{N}>0$ is a constant to be chosen, we decompose

$$
\Sigma_{p, N_{0}}^{d, \nu}(N)=F_{N}^{\prime} \oplus \Sigma_{p, N_{0}}^{d, \nu}\left(N, A_{N}\right), \Sigma_{p, N_{0}}^{\prime \prime d, \nu}(N)=F_{N}^{\prime \prime} \oplus \Sigma_{p, N_{0}}^{\prime \prime d, \nu}\left(N, A_{N}\right)
$$

where $F_{N}^{\prime}, F_{N}^{\prime \prime}$ is the subspace consisting of symbols $a$ satisfying $a\left(\cdot ; n_{0}, n_{p+1}\right) \equiv 0$ for $n_{0}+n_{p+1}>A_{N}$. By (ii) of Lemma 4.3.3, it is enough to construct

$$
\begin{gathered}
L_{N}^{-1}: \Sigma_{p, N_{0}}^{d, \nu}\left(N, A_{N}\right) \rightarrow \Sigma_{p, N_{0}}^{d, \nu+N_{0}}\left(N+1, A_{N}\right), \\
L_{N}^{-1}: \Sigma_{p, N_{0}}^{\prime \prime d, \nu}\left(N, A_{N}\right) \rightarrow \Sigma_{p, N_{0}}^{\prime \prime d-1, \nu+N_{0}}\left(N, A_{N}\right)
\end{gathered}
$$

for $A_{N}$ large enough. Recall decomposition (4.3.9) of $L$, and let us first construct an inverse $L_{0, N}^{-1}$ to $L_{0}$. We take $\tilde{a}$ respectively in $\Sigma_{p, N_{0}}^{d, \nu}\left(N, A_{N}\right)$ or $\Sigma_{p, N_{0}}^{\prime \prime d, \nu}\left(N, A_{N}\right)$ and look for $c^{1}$ in the right hand side of (4.3.26) with $L_{0}\left(c^{1}\right)=\tilde{a}$. We use expressions (4.3.12), (4.3.14) for $L_{0}\left(c^{1}\right)$. If we compose on the right with $J_{\epsilon_{p+1}}$ defined in (4.3.18) and evaluate $L_{0}\left(c^{1}\right)$ at $\Pi_{n^{\prime}}^{\ell^{\prime}} \epsilon^{\prime} U^{\prime}=\left(\Pi_{n_{1}}^{\ell_{1}, \epsilon_{1}} u_{1}, \ldots, \Pi_{n_{p}}^{\ell_{p}, \epsilon_{p}} u_{p}\right)$, we get respectively the equalities

$$
\begin{array}{r}
\tilde{c}_{D_{0}}^{1}\left(\Pi_{n^{\prime}}^{\ell^{\prime}, \epsilon^{\prime}} U^{\prime} ; n_{0}, n_{p+1}\right) J_{\epsilon_{p+1}}+\tilde{c}^{1}\left(\Pi_{n^{\prime}}^{\ell^{\prime}, \epsilon^{\prime}} U^{\prime} ; n_{0}, n_{p+1}\right)\left(D_{0}^{\prime}\left(n_{p+1}\right) \mp D_{0}^{\prime}\left(n_{0}\right)\right) J_{\epsilon_{p+1}} \\
=\tilde{a}\left(\Pi_{n^{\prime}}^{\ell^{\prime}}, \epsilon^{\prime} U^{\prime} ; n_{0}, n_{p+1}\right) J_{\epsilon_{p+1}} .
\end{array}
$$


Using (4.3.7) and (4.3.20), (4.3.23) we see that we may define $c^{1}$ by

$$
\begin{aligned}
& \tilde{c}^{1}\left(\Pi_{n^{\prime}} U^{\prime} ; n_{0}, n_{p+1}\right) \\
& \quad=-\sum_{\left(\ell_{1}, \epsilon_{1}\right), \ldots,\left(\ell_{p}, \epsilon_{p}\right), \epsilon_{p+1}} i F_{\mp}^{\ell^{\prime}, \epsilon}\left(n_{0}, \ldots, n_{p+1}\right)^{-1} \tilde{a}\left(\Pi_{n^{\prime}}^{\ell^{\prime}, \epsilon^{\prime}} U^{\prime} ; n_{0}, n_{p+1}\right) J_{\epsilon_{p+1}},
\end{aligned}
$$

where the sum is taken for $\ell_{1}, \ldots, \ell_{p}, \epsilon_{1}, \ldots, \epsilon_{p+1}$ in a set of bounded cardinal, and where

$$
F_{\mp}^{\ell^{\prime}, \epsilon}\left(n_{0}, \ldots, n_{p+1}\right)=\sum_{j=1}^{p} \epsilon_{j} \omega_{m}\left(n_{j}, \ell_{j}\right)+\epsilon_{p+1}\left(\omega_{m}\left(n_{p+1}\right) \mp \omega_{m}\left(n_{0}\right)\right) .
$$

It is enough to check that each term in the sum (4.3.27) belongs to the right hand side of (4.3.26). Observe that $F_{-}^{\ell^{\prime}, \epsilon}$ is a function of type (3.3.10) that satisfies (3.3.11): if $\left|n_{0}-n_{p+1}\right|$ is large relative to $\left|n^{\prime}\right|$, this follows from the fact that $\omega_{m}(n)=n+O(1 / n), n \rightarrow+\infty$. If $\left|n_{0}-n_{p+1}\right| \leq C\left|n^{\prime}\right|$ this is a consequence of Proposition 4.3.1. By inequalities (3.3.12), in the case of sign - in (4.3.27), we see that $\tilde{c}^{1}$ is a symbol in $\Sigma_{\substack{\prime \\ p, N_{0}}}^{d, \nu+N_{0}}\left(N+1, A_{N}\right)$ (taking eventually for $N_{0}$ a larger value than the one of (3.3.11)). In the case of $F_{+}^{\ell^{\prime}, \epsilon}$, we remark that it satisfies (3.3.13). So (3.3.12) will be controlled in terms of (3.3.14). This implies that for $\tilde{a} \in \Sigma_{p, N_{0}}^{\prime \prime d, \nu}\left(N, A_{N}\right)$, (4.3.27) defines a symbol $\tilde{c}^{1}$ in $\Sigma_{p, N_{0}}^{\prime \prime d-1, \nu+N_{0}}\left(N, A_{N}\right)$. Consequently we have defined a bounded inverse $L_{0, N}^{-1}$ to $L_{0}$, acting on the space (4.3.26). To define $L_{N}^{-1}$ as

$$
L_{N}^{-1}=\left(L_{0}\left(\mathrm{Id}+L_{0, N}^{-1} L_{1}\right)\right)^{-1}=\left(\operatorname{Id}+L_{0, N}^{-1} L_{1}\right)^{-1} L_{0, N}^{-1}
$$

we just need to check that the operator norm of $L_{0, N}^{-1} \circ L_{1}$ from $\Sigma_{p, N_{0}}^{\prime d, \nu+N_{0}}\left(N+1, A_{N}\right)$ (resp. $\Sigma_{p, N_{0}}^{\prime \prime d-\nu+N_{0}}\left(N, A_{N}\right)$ ) to itself is smaller than one if $A_{N}$ is large enough. But we have seen that $L_{1}$ sends $\Sigma_{p, N_{0}}^{d^{\prime}, \nu}(N)$ to $\Sigma_{p, N_{0}}^{-\infty, 0}(N)$ for any $d^{\prime}$. By definition (4.3.11) of $L_{1}$, the same is true for the $\Sigma^{\prime}$ or $\Sigma^{\prime \prime}$ spaces, so the operator norm of $L_{0, N}^{-1} \circ L_{1}$ on the above spaces is bounded from above by $C_{N} / A_{N}$, where $C_{N}>0$ is independent of $A_{N}$ (it suffices to extract from the gain on the order coming from $L_{1}$ a factor $\frac{1}{n_{0}+n_{p+1}} \leq \frac{1}{A_{N}}$ ). The conclusion follows for large enough $A_{N}$.

We shall also need a result, similar to Proposition 4.3.2, but for remainder operators.

Proposition 4.3.4. Let $d \in \mathbb{R}$, let $p \in \mathbb{N}$ an odd number and $\nu \in \mathbb{R}_{+}$. For every $\widetilde{M} \in \mathcal{R}_{p+1}^{d, \nu}$ there is $\widetilde{M}_{1} \in \mathcal{R}_{p+1}^{d, \nu+N_{0}}$ such that for any $u_{1}, \ldots, u_{p+1} \in \mathcal{E}$,

$$
\sum_{j=1}^{p+1} \widetilde{M}_{1}\left(u_{1}, \ldots, M_{0} u_{j}, \ldots, u_{p+1}\right)+M_{0}^{*} \widetilde{M}_{1}\left(u_{1}, \ldots, u_{p+1}\right)=\widetilde{M}\left(u_{1}, \ldots, u_{p+1}\right) .
$$


Proof. We extend $\widetilde{M}, \widetilde{M}_{1}$ as $\mathbb{C}$-multilinear maps, replace $u_{j}$ by $P_{0} u_{j}$ and compose on the left by $P_{0}^{*}$. Since $M_{0} P_{0}=P_{0} D_{0}$ and $D_{0}^{*}=-D_{0}$ we get

$$
\begin{array}{r}
\sum_{j=1}^{p+1} P_{0}^{*} \widetilde{M}_{1}\left(P_{0} u_{1}, \ldots, P_{0} D_{0} u_{j}, \ldots, P_{0} u_{p+1}\right)- \\
D_{0} P_{0}^{*} \widetilde{M}_{1}\left(P_{0} u_{1}, \ldots, P_{0} u_{p+1}\right) \\
=P_{0}^{*} \widetilde{M}\left(P_{0} u_{1}, \ldots, P_{0} u_{p+1}\right) .
\end{array}
$$

We use the notation (4.3.19). We compose on the left (4.3.29) with $\Pi_{n_{0}}^{\ell_{0}, \epsilon_{0}}$ and replace $u_{j}$ by $\Pi_{n_{j}}^{\ell_{j}, \epsilon_{j}} u_{j}$, for any possible values of $n_{0}, \ldots, n_{p+1}, \ell_{0}, \ldots, \ell_{p+1}, \epsilon_{0}, \ldots, \epsilon_{p+1}$. If $U=\left(u_{1}, \ldots, u_{p+1}\right), n=\left(n_{1}, \ldots, n_{p+1}\right), \ell=\left(\ell_{1}, \ldots, \ell_{p+1}\right), \epsilon=\left(\epsilon_{1}, \ldots, \epsilon_{p+1}\right)$ we set

$$
\Pi_{n}^{\ell, \epsilon} U=\left(\Pi_{n_{1}}^{\ell_{1}, \epsilon_{1}} u_{1}, \ldots, \Pi_{n_{p+1}}^{\ell_{p+1}, \epsilon_{p+1}} u_{p+1}\right)
$$

Using (4.3.20) we see that (4.3.29) may be written

$$
\Pi_{n_{0}}^{\ell_{0}, \epsilon_{0}} P_{0}^{*} \widetilde{M}_{1}\left(P_{0} \Pi_{n}^{\ell, \epsilon} U\right)=-i\left(\sum_{j=1}^{p+1} \epsilon_{j} \omega_{m}\left(n_{j}, \ell_{j}\right)-\epsilon_{0} \omega_{m}\left(n_{0}, \ell_{0}\right)\right)^{-1} \Pi_{n_{0}}^{\ell_{0}, \epsilon_{0}} P_{0}^{*} \widetilde{M}\left(\Pi_{n}^{\ell, \epsilon} P_{0} U\right)
$$

so that replacing $U$ by $P_{0}^{-1} U$,

$$
\begin{array}{r}
\Pi_{n_{0}} \widetilde{M}_{1}\left(\Pi_{n} U\right)=-i \sum_{\left(\ell_{0}, \epsilon_{0}\right), \ldots,\left(\ell_{p+1}, \epsilon_{p+1}\right)}\left(\sum_{j=1}^{p+1} \epsilon_{j} \omega_{m}\left(n_{j}, \ell_{j}\right)-\epsilon_{0} \omega_{m}\left(n_{0}, \ell_{0}\right)\right)^{-1} \\
\times\left(P_{0}^{*}\right)^{-1} \Pi_{n_{0}}^{\ell_{0}, \epsilon_{0}} P_{0}^{*} \widetilde{M}\left(\Pi_{n}^{\ell, \epsilon} U\right),
\end{array}
$$

where the sum is taken for $\ell_{0}, \ldots, \ell_{p+1}, \epsilon_{0}, \ldots, \epsilon_{p+1}$ staying in a bounded set of indices. By Proposition 4.3.1 the first factor in the sum is bounded from above by $C \mu\left(n_{0}, \ldots, n_{p+1}\right)^{N_{0}}$. If we use that $\widetilde{M}$ satisfies estimates of type (2.1.15) the same is true for $\widetilde{M}_{1}$, with $\nu$ replaced by $\nu+N_{0}$, since $\mu\left(n_{0}, \ldots, n_{p+1}\right) \leq \max _{2}\left(n_{1}, \ldots, n_{p+1}\right)$. This concludes the proof.

Proof of Theorem 1.1.1. We wrote equation (1.1.4) under the equivalent form (4.2.4) or (4.2.13). It is enough to show that there is $s_{0}$ large enough, such that if $s \geq s_{0}$, there is $C_{s}>0$ and $R_{0}>0$ so that, if $u(t, \cdot)$ is a solution of (4.2.4) defined on some interval $[0, T]$, with Cauchy data in $H^{s}$, one has for any $t \in[0, T]$,

$$
\|u(t, \cdot)\|_{H^{s}}^{2} \leq C_{s}\left[\|u(0, \cdot)\|_{H^{s}}^{2}+\int_{0}^{t}\|u(\tau, \cdot)\|_{H^{s_{0}}}^{r-1}\|u(\tau, \cdot)\|_{H^{s}}^{2} d \tau\right]
$$

as long as $\|u(t, \cdot)\|_{H^{s_{0}}} \leq R_{0}$. Actually, applying (4.3.30) with $s=s_{0}$, assuming $\|u(0, \cdot)\|_{H^{s_{0}}} \leq \epsilon$ and taking $R_{0}=\left(2 C_{s_{0}}\right)^{1 / 2} \epsilon$, we see that we may extend the solution as an $H^{s_{0}}$ function up to time $t_{0}=\frac{1}{2 C_{s_{0}}} R_{0}^{-r+1}=c_{s_{0}} \epsilon^{-r+1}$. If the Cauchy data are $H^{s}$ with $s \geq s_{0}$, the solution is also in $H^{s}$ on the same interval. It will be bounded in $H^{s}$ on an interval of length $\frac{1}{2 C_{s}} R_{0}^{-r+1}=c_{s} \epsilon^{-r+1}$.

Because of (4.2.16), we may in (4.3.30) replace $\|u(t, \cdot)\|_{H^{s}}^{2}$ by $\Theta_{0}^{s}(u(t, \cdot))$. Moreover, if on the right hand side of (4.2.17) we replace $\mathrm{Op}(c(u ; \cdot))$ by

$$
\frac{1}{2}\left[\mathrm{Op}(c(u ; \cdot)) \operatorname{Op}\left(\left(1+a_{\chi}\right)(u ; \cdot)\right)+\mathrm{Op}\left(\left(1+a_{\chi}\right)(u ; \cdot)\right) \operatorname{Op}(c(u ; \cdot))\right]
$$

we make appear an error that may be written, by Corollary 3.3.5 (i), as $\langle\mathrm{Op}(e(u ; \cdot)) \tilde{u}, \tilde{u}\rangle$, where $e$ is a symbol in $\widetilde{\Sigma}_{1}^{2 s, \nu^{\prime}}$ for some $\nu^{\prime}$ independent of $s$, of 
valuation $v(e) \geq 2 \kappa \geq r-1$. Consequently, by Proposition 2.1.3 and (4.2.22),

$$
\|\mathrm{Op}(e(u ; \cdot)) \tilde{u}\|_{H^{-s}} \leq C\|u\|_{H^{s_{0}}}^{r-1}\|u\|_{H^{s}}
$$

if $s \geq s_{0}$, and $s_{0}$ is large enough relative to $\nu^{\prime}$. We thus see that if we modify the definition of $\Theta_{0}^{s}$ replacing in (4.2.17) $\mathrm{Op}(c(u ; \cdot))$ by (4.3.31), we still get a quantity equivalent to $\|u\|_{H^{s}}^{2}$ when $\|u\|_{H^{s_{0}}}$ is small enough. We may thus assume from now on that

$$
\Theta_{0}^{s}(u)=\frac{1}{2}\left\langle\left[\operatorname{Op}\left(c^{0}(u ; \cdot)\right) \operatorname{Op}\left(\left(1+a_{\chi}\right)(u ; \cdot)\right)+\operatorname{Op}\left(\left(1+a_{\chi}\right)(u ; \cdot)\right)^{*} \operatorname{Op}\left(c^{0}(u ; \cdot)\right)\right] \tilde{u}, \tilde{u}\right\rangle
$$

for a scalar selfadjoint symbol $c^{0} \in \widetilde{\Sigma}_{1}^{2 s, \nu}$, and $c^{0}$ satisfying condition $C(\kappa, r)$ of Definition 3.3.2. We may decompose $c^{0}$ as a finite sum of homogeneous symbols $c_{p}^{0} \in$ $\Sigma_{p, 1}^{2 s, \nu}$. Remark that the contributions coming from the components homogeneous of degree $p \geq r-1$ give again a contribution to $\Theta_{0}^{s}(u)$ which is $O\left(\|u\|_{H^{s_{0}}}^{r-1}\|u\|_{H^{s}}^{2}\right)$. Modifying again the definition of $\Theta_{0}^{s}$, we may thus assume

$$
c^{0}=\sum_{p=0}^{r-2} c_{p}^{0}
$$

Since $c^{0}$ satisfies $C(\kappa, r)$, terms indexed by even $p$ 's in the above sum are zero. We compute the time derivative of (4.3.32) by applying Proposition 4.2.4 to each homogeneous component. Remark that assumptions (4.2.34), (4.2.35) are satisfied since $c^{0}$ is scalar and selfadjoint. We get, by (4.2.39),

$$
\begin{aligned}
\frac{d}{d t} \Theta_{0}^{s}(u(t, \cdot))= & \left\langle\mathrm{Op}\left(c_{M_{0}}^{0}(u ; \cdot)\right) \tilde{u}, \tilde{u}\right\rangle+\left\langle\left[\mathrm{Op}\left(c^{0}(u ; \cdot)\right) D_{0}+D_{0}^{*} \mathrm{Op}\left(c^{0}(u ; \cdot)\right)\right] \tilde{u}, \tilde{u}\right\rangle \\
& +\left\langle\mathrm{Op}\left(e^{0}(u ; \cdot)\right) \tilde{u}, \tilde{u}\right\rangle+2 \operatorname{Re}\left\langle R^{0}(u), u\right\rangle,
\end{aligned}
$$

where $e^{0} \in \widetilde{\Sigma}_{N_{0}}^{2 s, \nu^{\prime}}, R^{0} \in \widetilde{\mathcal{R}}^{2 s, \nu^{\prime}}$ for some $\nu^{\prime}$ independent of $s$, and with $v\left(e^{0}\right) \geq \kappa$, $v\left(R^{0}\right) \geq \kappa+1$. Moreover $e^{0}$ is selfadjoint and these symbols and operators satisfy condition $C(\kappa, r)$. Since $c^{0}$ is scalar and $D_{0}^{*}=-D_{0}$, we get from Corollary 3.3.5 (ii) that the second duality bracket may be written as $\langle\mathrm{Op}(b(u ; \cdot)) \tilde{u}, \tilde{u}\rangle$ for a symbol $b \in \widetilde{\Sigma}_{1}^{2 s, \nu}$ for some $\nu$ independent of $s$. Moreover, since $c^{0}$ satisfies condition $C(\kappa, r)$, $c_{M_{0}}^{0}$ and $b$ have valuation greater than or equal to $\kappa$, and also satisfy $C(\kappa, r)$. We may thus write

$$
\frac{d}{d t} \Theta_{0}^{s}(u(t, \cdot))=\langle\operatorname{Op}(g(u ; \cdot)) \tilde{u}, \tilde{u}\rangle+2 \operatorname{Re}\left\langle R^{0}(u), u\right\rangle
$$

for a new symbol $g \in \widetilde{\Sigma}_{1}^{2 s, \nu}$ with $v(g) \geq \kappa, g$ satisfying condition $C(\kappa, r)$. In particular, the homogeneous components of order $p$ of $g$ with $\kappa \leq p<r-1$ vanish if $p$ is even. Moreover we may assume $g$ is selfadjoint. For odd $p, \kappa \leq p<r-1$, we decompose the corresponding contribution $g_{p}$ as $g_{p}^{\prime}+g_{p}^{\prime \prime}$, where $g_{p}^{\prime}$ satisfies assumption $\left(H_{D}\right)$ and $g_{p}^{\prime \prime}$ satisfies $\left(H_{N D}\right)$. By Proposition 4.3.2 for each such $p$, we may find $c_{p}^{1 \prime} \in \Sigma_{p, N_{0}}^{2 s, \nu^{\prime}}, c_{p}^{1 \prime \prime} \in \Sigma_{p, N_{0}}^{2 s-1, \nu^{\prime}}$ for some $\nu^{\prime}$ independent of $s$, such that (4.3.5) holds true for $c_{p}^{1}=c_{p}^{1 \prime}+c_{p}^{1 \prime \prime}$, when its right hand side is replaced by $g_{p}$. In particular, these $c_{p}^{1}$ have the structure (4.2.34), (4.2.35) which allows us to apply 
Proposition 4.2.4. More precisely, define

$$
\begin{aligned}
\Theta_{1}^{s}(u)=\frac{1}{2} \sum_{\substack{\kappa \leq p<r-1 \\
p \text { odd }}}\left\langle\left[\operatorname{Op}\left(c_{p}^{1}(u ; \cdot)\right) \operatorname{Op}\left(\left(1+a_{\chi}\right)(u ; \cdot)\right)\right.\right. & \\
& \left.\left.+\operatorname{Op}\left(\left(1+a_{\chi}\right)(u ; \cdot)\right)^{*} \operatorname{Op}\left(c_{p}^{1}(u ; \cdot)\right)\right] \tilde{u}, \tilde{u}\right\rangle .
\end{aligned}
$$

By (4.2.39) and (4.3.5) we have

$$
\frac{d}{d t} \Theta_{1}^{s}(u(t, \cdot))=\langle\mathrm{Op}(g(u ; \cdot)) \tilde{u}, \tilde{u}\rangle+\left\langle\mathrm{Op}\left(f^{0}(u ; \cdot)\right) u, u\right\rangle+2 \operatorname{Re}\left\langle S^{0}(u), u\right\rangle,
$$

where $f^{0} \in \widetilde{\Sigma}_{N_{0}}^{2 s, \nu^{\prime}}, S^{0} \in \widetilde{\mathcal{R}}^{2 s, \nu^{\prime}}$ for some $\nu^{\prime}$ independent of $s$, with $v\left(f^{0}\right) \geq 2 \kappa$, $v\left(S^{0}\right) \geq 2 \kappa+1$. (We used again 4.2.20) to express $\tilde{u}$ in terms of $u$ in the last but one term coming from (4.2.39).)

Let us define also a perturbation to get rid of the $\left\langle R^{0}(u), u\right\rangle$ term in (4.3.33). We may decompose $R^{0}=R^{\prime 0}+\tilde{R}^{0}$ with $R^{\prime 0}=\sum_{\kappa \leq p<r-1} R_{p+1}^{0}$ and $\tilde{R}^{0} \in \widetilde{\mathcal{R}}^{2 s, \nu}$ of valuation greater than or equal to $r$, and where $R_{p+1}^{0} \in \mathcal{R}_{p+1}^{2 s, \nu^{\prime}}$ and the sum is indexed by odd $p$ (since $R^{0}$ satisfies condition $C(\kappa, r)$ ). Define $\widetilde{M}_{p+1}$ as the solution of equation (4.3.28), when the right hand side is replaced by $R_{p+1}^{0}$. Then $\widetilde{M}_{p+1} \in \mathcal{R}_{p+1}^{2 s, \nu^{\prime}+N_{0}}$ and if we set

$$
\Theta_{2}^{s}(u)=2 \operatorname{Re} \sum_{\substack{\kappa \leq p<r-1 \\ p \text { odd }}}\left\langle\widetilde{M}_{p+1}(u, \ldots, u), u\right\rangle,
$$

it follows from Proposition 4.2.5 that

(4.3.35)

$$
\frac{d}{d t} \Theta_{2}^{s}(u(t, \cdot))=2 \operatorname{Re}\left[\left\langle R^{\prime 0}(u), u\right\rangle+\left\langle R^{1}(u), u\right\rangle+\sum_{\substack{\kappa \leq p<r-1 \\ p \text { odd }}}\left\langle\widetilde{M}_{p+1}(u, \ldots, u), R_{p+1}^{2}(u)\right\rangle\right]
$$

where $R^{1} \in \widetilde{\mathcal{R}}^{2 s+1, \nu^{\prime \prime}}, R_{p+1}^{2} \in \widetilde{\mathcal{R}}^{0, \nu^{\prime \prime}}$ for some $\nu^{\prime \prime}$ independent of $s$ and $v\left(R^{1}\right) \geq$ $2 \kappa+1 \geq r, v\left(R_{p+1}^{2}\right) \geq \kappa+1$. Combining (4.3.33), (4.3.34), (4.3.35) we get

$$
\begin{aligned}
\frac{d}{d t}\left[\Theta_{0}^{s}(u(t, \cdot))-\Theta_{1}^{s}(u(t, \cdot))-\Theta_{2}^{s}(u(t, \cdot))\right] & \\
& =-\left\langle\operatorname{Op}\left(f^{0}(u ; \cdot)\right) u, u\right\rangle+2 \operatorname{Re}\left\langle\tilde{R}^{0}(\tilde{u})-S^{0}(u)-R^{1}(u), u\right\rangle \\
& -2 \operatorname{Re} \sum_{\substack{\kappa \leq p<r-1 \\
p \text { odd }}}\left\langle\widetilde{M}_{p+1}(u, \ldots, u), R_{p+1}^{2}(u)\right\rangle .
\end{aligned}
$$

The right hand side is bounded from above by

$$
\begin{array}{r}
C\left[\left\|\operatorname{Op}\left(f^{0}(u ; \cdot)\right) u\right\|_{H^{-s}}\|u\|_{H^{s}}+\left\|\tilde{R}^{0}(\tilde{u})-S^{0}(u)-R^{1}(u)\right\|_{H^{-s}}\|u\|_{H^{s}}\right. \\
\left.+\sum_{\substack{\kappa \leq p<r-1 \\
p \text { odd }}}\left\|\widetilde{M}_{p+1}(u, \ldots, u)\right\|_{H^{-s}}\left\|R_{p+1}^{2}(u)\right\|_{H^{s}}\right] .
\end{array}
$$

By Proposition 2.1.3, and using that $v\left(f^{0}\right) \geq 2 \kappa$, there is some $s_{0}$, depending on $\nu^{\prime}$ but not on $s$, such that when $s \geq s_{0}$ the first term in (4.3.37) is bounded by $C\|u\|_{H^{s_{0}}}^{2 \kappa}\|u\|_{H^{s}}^{2}$, as long as $\|u\|_{H^{s_{0}}} \leq 1$. In the second term of (4.3.37), $S^{0}, R^{1}, \tilde{R}^{0}$ belong to $\widetilde{\mathcal{R}}^{2 s+1, \nu^{\prime \prime}}$ for some $\nu^{\prime \prime}$ independent of $s$, and have valuation greater than or equal to $r$. By Lemma 2.1.7 and inequality (2.1.19), for $s$ large enough relative to 
$\nu^{\prime \prime}$, the second term in (4.3.37) is controlled by $C\|u\|_{H^{s_{0}}}^{r-1}\|u\|_{H^{s}}^{2}$. Since $R_{p+1}^{2} \in \widetilde{\mathcal{R}}^{0, \nu^{\prime \prime}}$ with $v\left(R_{p+1}^{2}\right) \geq \kappa+1$, Lemma2.1.7implies $\left\|R_{p+1}^{2}(u)\right\|_{H^{s}} \leq C\|u\|_{H^{s_{0}}}^{\kappa}\|u\|_{H^{s}}$ for some $s_{0}$ large enough. Since $\widetilde{M}_{p+1} \in \mathcal{R}_{p+1}^{2 s, \nu^{\prime \prime}}$ with $\nu^{\prime \prime}$ independent of $s$ and $p+1 \geq \kappa+1$, the same lemma gives the estimate $\left\|\widetilde{M}_{p+1}(u, \ldots, u)\right\|_{H^{-s}} \leq C\|u\|_{H^{s_{0}}}^{\kappa}\|u\|_{H^{s}}$ if $s_{0}$ is large enough (independently of $s$ ), and $\|u\|_{H^{s_{0}}} \leq 1$. Finally we get for (4.2.34) an upper bound in terms of

$$
C\|u\|_{H^{s} 0}^{r-1}\|u\|_{H^{s}}^{2}
$$

using that $2 \kappa \geq r-1$. It then follows from (4.3.36) that for $t \geq 0$,

$$
\begin{aligned}
& \Theta_{0}^{s}(u(t, \cdot))-\Theta_{1}^{s}(u(t, \cdot))-\Theta_{2}^{s}(u(t, \cdot)) \\
& \leq \Theta_{0}^{s}(u(0, \cdot))-\Theta_{1}^{s}(u(0, \cdot))-\Theta_{2}^{s}(u(0, \cdot))+C \int_{0}^{t}\|u(\tau, \cdot)\|_{H^{s}}^{r-1}\|u(\tau, \cdot)\|_{H^{s}}^{2} d \tau
\end{aligned}
$$

when $s \geq s_{0}$ is large enough and when for $0 \leq t^{\prime} \leq t,\left\|u\left(t^{\prime}, \cdot\right)\right\|_{H^{s_{0}}} \leq 1$. Again by Proposition 2.1.3 and Lemma 2.1.7, we get when $\|u(t, \cdot)\|_{H^{s_{0}}} \leq 1$,

$$
\left|\Theta_{1}^{s}(u(t, \cdot))\right|+\left|\Theta_{2}^{s}(u(t, \cdot))\right| \leq C\|u(t, \cdot)\|_{H^{s_{0}}}^{\kappa}\|u(t, \cdot)\|_{H^{s}}^{2} .
$$

Inequality (4.3.30) follows from (4.3.38), (4.3.39) when $\left\|u\left(t^{\prime}, \cdot\right)\right\|_{H^{s_{0}}}$ stays small enough on the interval $[0, t]$. This concludes the proof.

\section{REFERENCES}

[1] D. Bambusi: Birkhoff normal form for some nonlinear PDEs, Comm. Math. Phys. 234 (2003), no. 2, 253-285. MR 1962462 (2003k:37121)

[2] D. Bambusi, J.-M. Delort, B. Grébert and J. Szeftel: Almost global existence for Hamiltonian semi-linear Klein-Gordon equations with small Cauchy data on Zoll manifolds, Comm. Pure Appl. Math. 60 (2007), no. 11, 1665-1690. MR2349351

[3] D. Bambusi and B. Grébert: Birkhoff normal form for partial differential equations with tame modulus, Duke Math. J. 135 (2006), no. 3, 507-567. MR2272975 (2007j:37124)

[4] J.-M. Bony: Calcul symbolique et propagation des singularités pour les équations aux dérivées partielles non linéaires, Ann. Sci. École Norm. Sup. (4) 14 (1981), no. 2, 209-246. MR631751 (84h:35177)

[5] J. Bourgain: Construction of approximative and almost periodic solutions of perturbed linear Schrödinger and wave equations, Geom. Funct. Anal. 6 (1996), no. 2, 201-230. MR1384610 (97f:35013)

[6] J. Bourgain: On the growth in time of higher Sobolev norms of smooth solutions of Hamiltonian PDE, Internat. Math. Res. Notices (1996), no. 6, 277-304. MR1386079 (97k:35016)

[7] J. Colliander, M. Keel, G. Staffilani, H. Takaoka and T. Tao: Resonant decompositions and the I-method for cubic nonlinear Schrödinger equation on $\mathbb{R}^{2}$, Discrete Contin. Dyn. Syst. 21 (2008), 665-686. MR2399431

[8] J.-M. Delort: Temps d'existence pour l'équation de Klein-Gordon semi-linéaire à données petites périodiques, Amer. J. Math. 120 (1998), no. 3, 663-689. MR1623424 (99d:35108)

[9] J.-M. Delort and J. Szeftel: Long-time existence for small data nonlinear Klein-Gordon equations on tori and spheres, Internat. Math. Res. Notices (2004), no. 37, 1897-1966. MR2056326 (2005k:35281)

[10] J.-M. Delort and J. Szeftel: Long-time existence for semi-linear Klein-Gordon equations with small Cauchy data on Zoll manifolds, Amer. J. Math. 128 (2006), no. 5, 1187-1218. MR2262173 (2007i:58039)

[11] J.-M. Delort and J. Szeftel: Bounded almost global solutions for non Hamiltonian semilinear Klein-Gordon equations with radial data on compact revolution hypersurfaces, Ann. Inst. Fourier (Grenoble) 56 (2006), no. 5, 1419-1456. MR.2273861(2008c:35191)

[12] B. Grébert: Birkhoff normal form and Hamiltonian PDEs, Partial differential equations and applications, 1-46, Sémin. Congr. 15, Soc. Math. France, Paris, 2007. MR2352816 
[13] L. Hörmander: Lectures on nonlinear hyperbolic differential equations, Mathématiques \& Applications 26, Springer-Verlag, Berlin, (1997), viii+289 pp. MR,1466700 (98e:35103)

[14] V. Marchenko: Sturm-Liouville operators and applications, Operator Theory: Advances and Applications, 22. Birkhäuser Verlag, Basel (1986), xii+367 pp. MR897106 (88f:34034)

[15] J. Shatah: Normal forms and quadratic nonlinear Klein-Gordon equations, Comm. Pure Appl. Math. 38 (1985), 685-696. MR803256 (87b:35160)

Université Paris 13, Institut Galilée, CNRS, UMr 7539, Laboratoire Analyse, Géométrie et Applications, 99, Avenue J.-B. Clément, F-93430 Villetaneuse, France

E-mail address: delort@math.univ-paris13.fr 